\title{
MICRO-SCALE HEAT TRANSFER SIMULATION ON WATER WITH MOLECULAR DYNAMICS AND HYBRID APPROACH
}

\begin{tabular}{c} 
A Dissertation \\
Presented to \\
the Faculty of the Graduate School \\
University of Missouri-Columbia \\
\hline
\end{tabular}

In Partial Fulfillment

of the Requirements for the Degree

Doctor of Philosophy

by

Yijin Mao

Dr. Yuwen Zhang, Dissertation Supervisor

July 2014 
The undersigned, appointed by the dean of the Graduate School, have examined the dissertation entitled

\section{MICRO-SCALE HEAT TRANSFER SIMULATION ON WATER WITH MOLECULAR DYNAMICS AND HYBRID APPROACH}

presented by Yijin Mao, a candidate for the degree of doctor of philosophy, and hereby certify that, in their opinion, it is worthy of acceptance.

Professor Yuwen Zhang

Professor Jinn-Kuen Chen

Professor Gary Solbrekken

Professor Hongbin "Bill" Ma

Professor Stephen Montgomery-Smith 


\section{ACKNOWLEDGEMENT}

I would like to express my deepest appreciation to my supervisor, Yuwen Zhang, who continuously directs me in every stage of my $\mathrm{PhD}$ work with great patience. His spirit of hard working and strictness in academy impresses me much and this will be a great treasure in all my life. Without his persistent guidance and support, this dissertation would not be possible.

Great gratitude is also given to my parents who love and encourage me by standing by me to overcome all difficulties in study and life. Their sacrificial love is my drive to reach my goal in all my life. 


\section{TABLE OF CONTENTS}

ABSTRACT

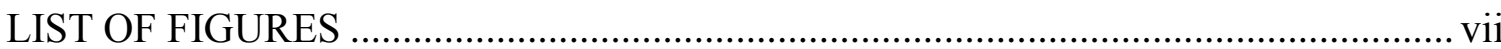

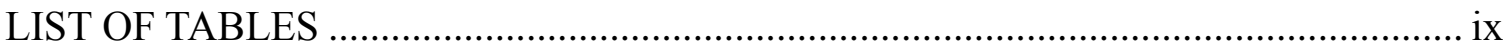

NOMENCLATURE

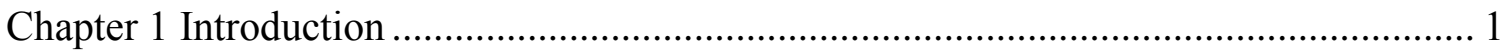

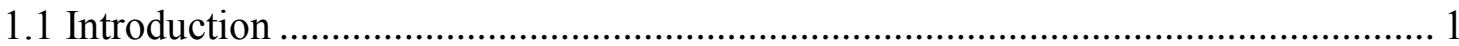

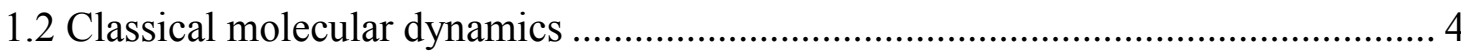

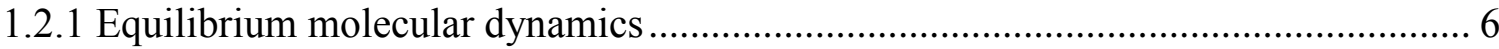

1.2.2 Non-equilibrium molecular dynamics simulation ................................................. 7

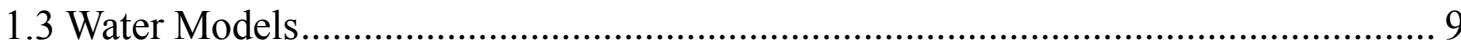

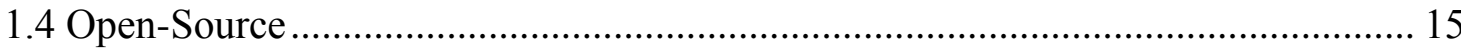

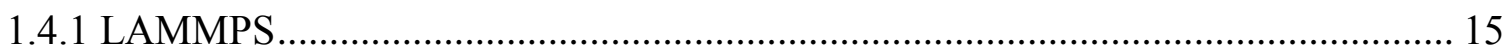

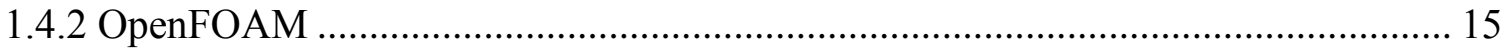

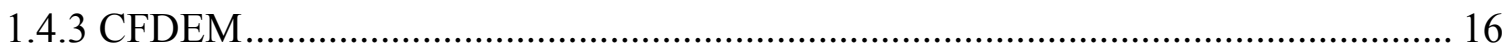

1.5 Dissertation Objectives ................................................................................... 16

Chapter 2 Thermal Conductivity, Shear Viscosity and Specific Heat of Rigid Water

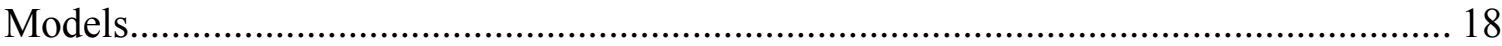

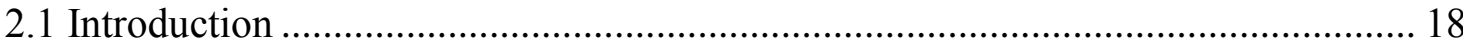

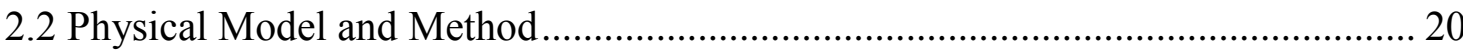

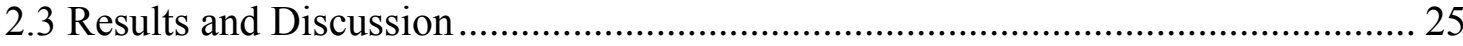

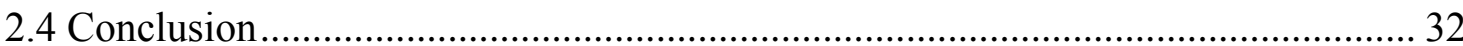

Chapter 3 Prediction of the Temperature-Dependent Thermal Conductivity and Shear

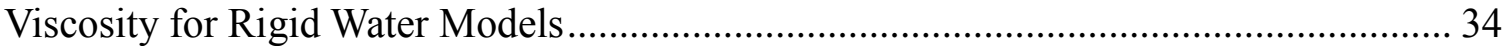

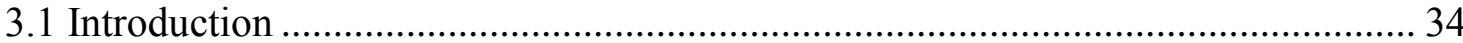

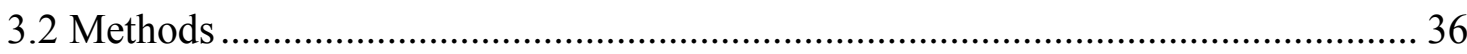

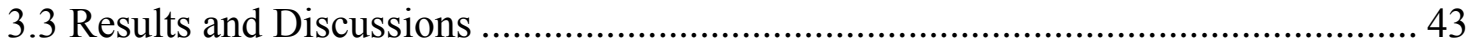

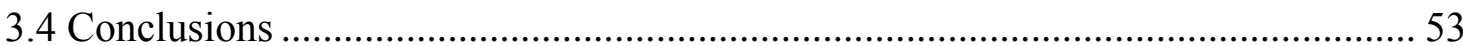

Chapter 4 Nonequilibrium Molecule Dynamics Simulations of Nanobubble Growth and Annihilation in Liquid Water ........................................................................................ 54

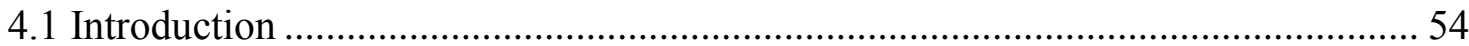

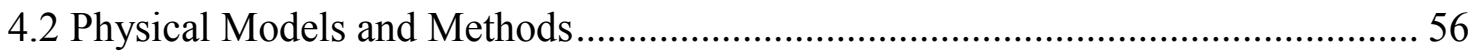

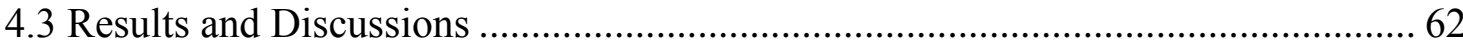

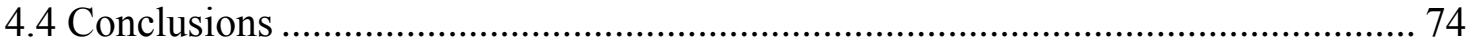


Chapter 5 Molecular Dynamic Study on Contact Angle of Water Droplet on a Single-Wall Carbon Nanotube (SWCNT) Plate............................................................................... 76

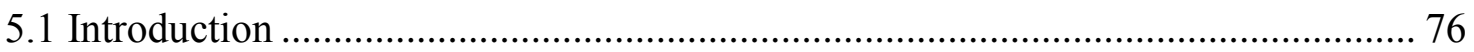

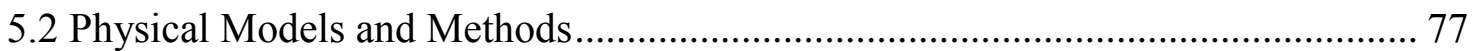

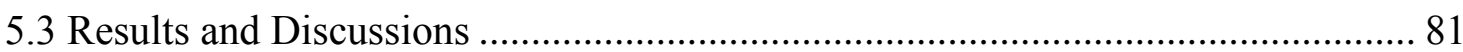

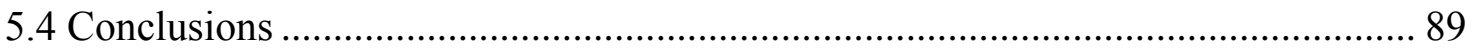

Chapter 6 Molecular Dynamics Simulation on Rapid Boiling of Water on a Hot Copper

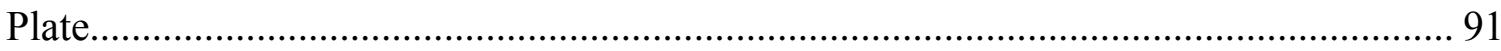

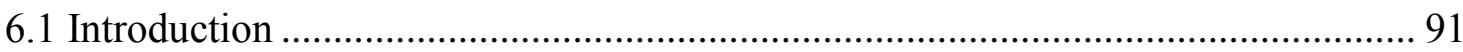

6.2 Physical Models and Methods............................................................................... 92

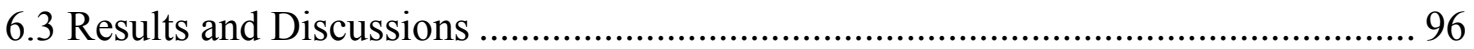

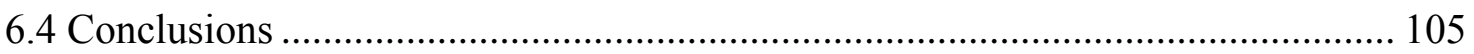

Chapter 7 Atomistic-Continuum Hybrid Simulation of Heat Transfer between Argon Flow and Copper Plates ……………………………........................................... 107

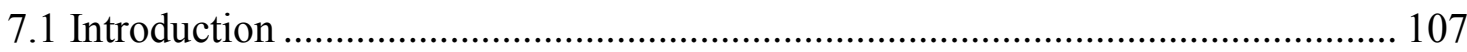

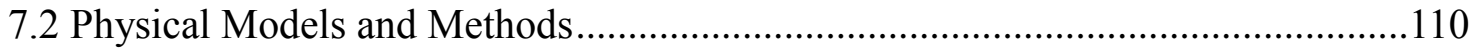

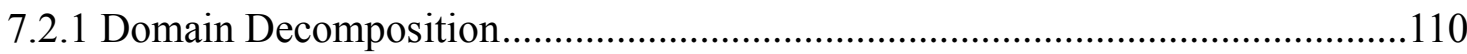

7.2.2 Governing equations for continuum region $(\mathrm{C})$................................................111

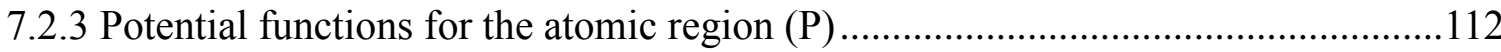

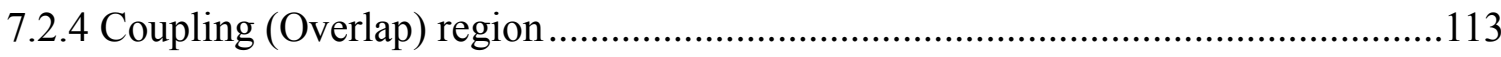

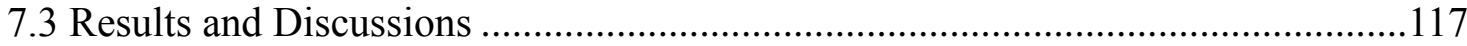

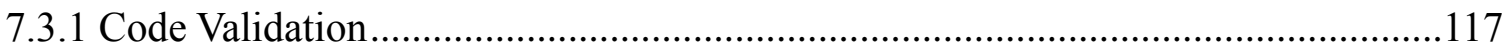

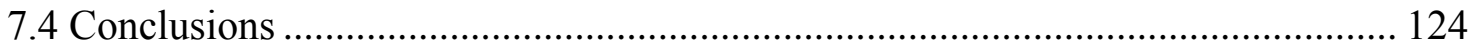

Chapter 8 Conclusions and future work............................................................... 126

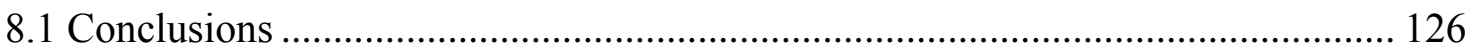

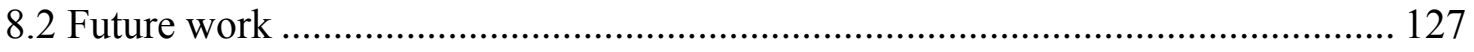

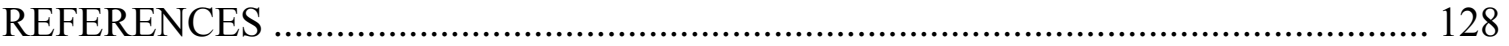

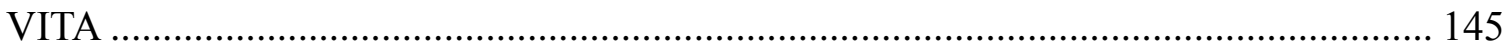




\begin{abstract}
Water is most widely existing liquid in the earth; beyond all question, it takes a very important role in human daily life and many applications in industrial field. The heat transfer phenomena have drawn a great attention in the past decades. In the thesis, classical molecular dynamic simulations are carried out to study thermal and dynamic properties, including thermal conductivities, specific heat capacity, and shear viscosity. Though water molecule only has three charged atoms, but a number of water molecule models exist in the literature field. In this thesis, several rigid water molecule models are selected to perform the simulation. Then a non-equilibrium molecular dynamic is conducted to study nano-bubble growth and annihilation in the liquid water with rigid TIP3P. A nano-bubble is successfully generated, and pressure and surface tension of the bubble is obtained. Contact angle of a water droplet is also getting attention from microcooling engineers. A non-equilibrium molecular dynamics on contact angle of nano-size water droplet on a single wall carbon nanotube plate is performed. The gap distance between nano-tube affects the contact angle somehow, and a critical distance value that achieve largest contact angle is found. Rapid boiling phenomena of thin water film on a hot copper plate is also studied due to its application in various industrial fields, such as laser cleaning, thermal ink-jet printer, medical surgery. The entire process of the boiling phenomena is observed, and a piston-like motion of the film is analyzed. To the end, due to the size limitation of classical molecular dynamics, a hybrid simulation coupling molecular dynamic and computational fluid dynamic is developed to extend the
\end{abstract}


simulation size to micron based on open-source code LIGGGHTS and OpenFOAM. Heat transfer coefficient between argon flow and cold copper plate is studied and it is found that heat transfer coefficient can reach a very high value. In the future work, argon fluid could be replaced with normal liquid water molecules, and some similar or more complicated simulation can be performed within current framework. 


\section{LIST OF FIGURES}

Figure 1-1 Four types of water molecules[14]................................................. 10

Figure 2-1 Established temperature gradient for TIP5P-Ew water system at 318K. 26

Figure 2-2 Established velocity gradient for TIP4P/2005 water system at $318 \mathrm{~K} . . . .29$

Figure 2-3 Relation between enthalpy and temperature for SPC/E water system.... 30

Figure 2-4 Relation between internal energy and temperature for TIP4P-Ew water

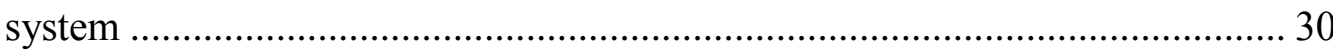

Figure 3-1 Schematic of reverse non-equilibrium molecular dynamics (RNEMD). 37

Figure 3-2 Temperature profiles in simulation box for each rigid model at temperatures ranging from $283 \mathrm{~K}$ to $363 \mathrm{~K}$......................................................... 44

Figure 3-3 Thermal conductivities trends predicted by eight rigid models .............. 45

Figure 3-4 velocity profiles (in $\mathrm{x}$ component) for each rigid model at temperatures

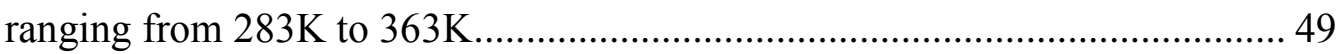

Figure 3-5 Shear viscosities trends predicted by eight rigid models ........................ 52

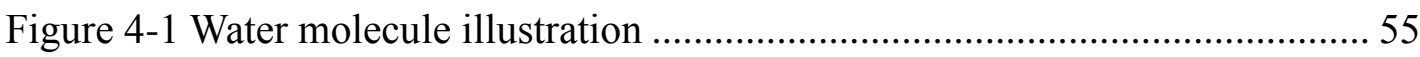

Figure 4-2 Molecule number estimation in one molecule thinkness layer ................ 62

Figure 4-3 Snapshots of the bubble during the period from 0 ps to $5.06 \mathrm{ps}$.............. 64

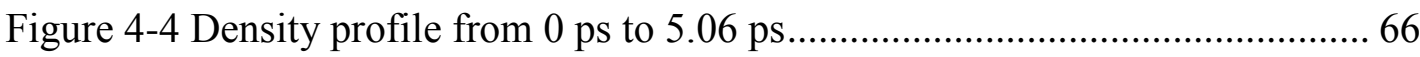

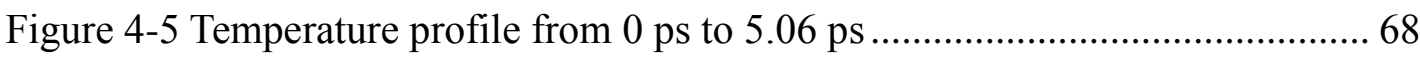

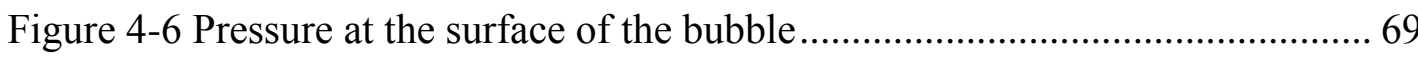

Figure 4-7 Bubble radius variation in water (S refer to Sample Point) ..................... 70

Figure 4-8 Surface tension profiles obtained by assuming the validity of the

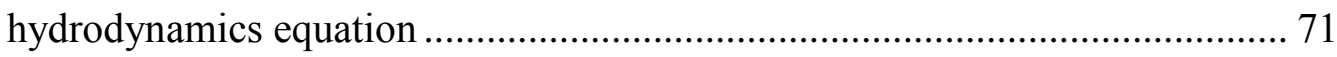

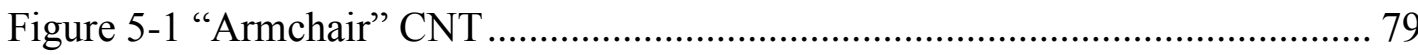

Figure 5-2 Comparison of wettability of CNT with different separation distance ... 83

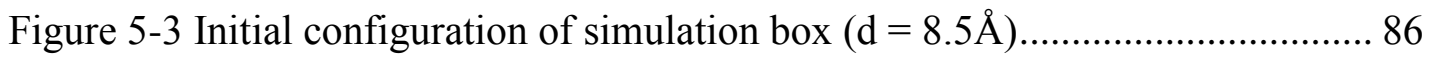

Figure 5-4 A comparison between two cases with/without quadrupole term $(\mathrm{d}=13$

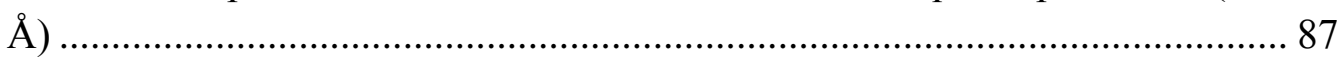

Figure 5-5 water molecule distribution with carbon nanotube plates $(\mathrm{d}=13 \AA) \ldots . . .88$

Figure 6-1 Structures of the copper plate, artificial harmonic bond are created by connecting neighbor atom within a distance of $2.56 \AA$................................... 94

Figure 6-2Temperature variation of water and hot copper plate................................ 97

Figure 6-3 Snapshots of water molecule distribution through time $10 \mathrm{ps}$ to $400 \mathrm{ps} 98$

Figure 6-4 z-component of COM associated to water molecules within the

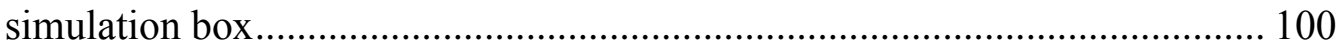

Figure 6-5 One-dimensional spatial density distribution at various times ............. 102

Figure 6-6 Top views of water molecules distribution on the surface of the copper

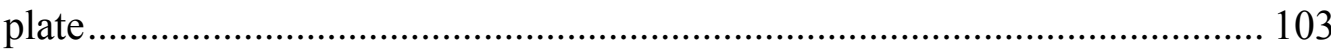

Figure 6-7 Variation of one dimensional spatial density distribution with time..... 104

Figure 6-8 Temperature variation in three regions................................................ 105 
Figure 7-1 Schematic of domain decomposition ...............................................111

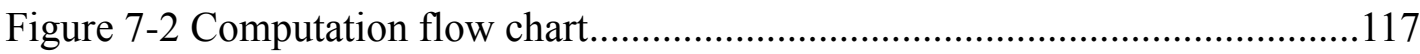

Figure 7-3 Configuration of test cases and final velocity profiles............................119

Figure 7-4 Configuration of test cases and final temperature profiles.................... 120

Figure 7-5 Temperature and velocity distribution along with y axis (Couette flow with heat transfer) ................................................................................ 122

Figure 7-6 Heat flux variation along with simulation time-step............................. 124 


\section{LIST OF TABLES}

Table 1-1 Parameters for different types of water models[14] .................................11

Table 1-2 properties predicted by different water models[14] ............................... 14

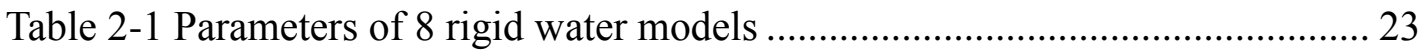

Table 2-2 Comparison of results from different Kspace Solver ............................... 25

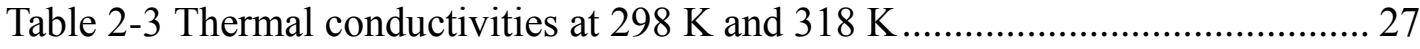

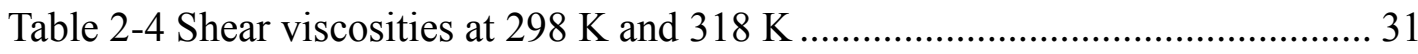

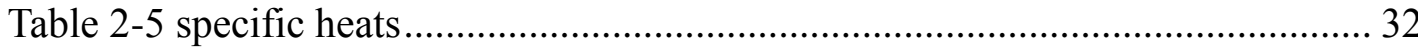

Table 3-1 Parameters of 8 rigid water models ........................................................ 41

Table 3-2 Comparison of results from different Kspace Solver ............................. 43

Table 3-3 Thermal conductivities of different model at various temperatures ( $\mathrm{W}$ m-1

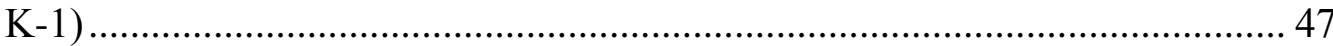

Table 3-4 Shear viscosities of different model at various temperatures $(\mathrm{mPa} \cdot \mathrm{s})$...... 50

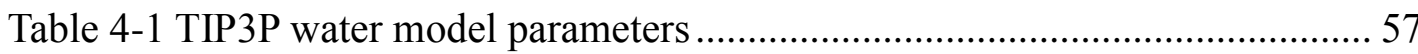

Table 5-1 potential function parameters of water related interaction ...................... 78

Table 5-2 potential function parameters of carbon related interaction .................... 79

Table 5-3 potential function parameters of water-carbon interaction ....................... 80

Table 5-4 Minimum distance between water molecule and single CNT at equilibrium

Table 5-5 contact angles of droplet sitting on various CNT plate with/without

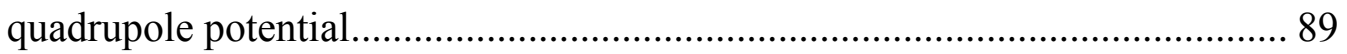

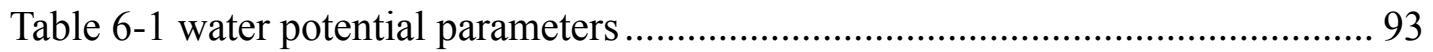




\section{NOMENCLATURE}

\section{Nomenclature}

$d \quad$ thickness of phase transition (lattice constant, $\AA$, chapter 6)

e internal energy, $\mathrm{J}$

$\mathbf{f}_{\mathrm{i}} \quad$ force acting on $\mathrm{i}^{\text {th }}$ atom

$h \quad$ heat transfer coefficient

$j_{i} \quad$ velocity gradient, $1 / \mathrm{s}$

$k \quad$ thermal conductivity, $\mathrm{W} / \mathrm{m}-\mathrm{K}$ (spring constant, $\mathrm{eV} / \AA^{2}$, Chapter 6)

$k_{\mathrm{B}} \quad$ Boltzmann constant

$k_{C} \quad$ electrostatic constant, $\AA \cdot \mathrm{kcal} / \mathrm{mol}$

$m \quad$ molar mass, g/mol (Coupling interval, Chapter 7)

$m_{\mathrm{i}} \quad$ mass of atom $\mathrm{i}^{\text {th }}$

$n \quad$ pairs number of selected molecules

$p \quad$ pressure, $\mathrm{Pa}$

$q \quad$ electron charge, $\mathrm{e}$

$q^{\prime \prime} \quad$ heat flux, $\mathrm{W} / \mathrm{m}^{2}$

$\mathrm{r}_{\mathrm{c}} \quad$ cutoff distance $(\AA)$

$\mathbf{r}_{\mathrm{i}} \quad$ position vector of atom $\mathrm{i}$

$r_{i j} \quad$ distance between two atoms $i$ and $j, \AA$

$\mathbf{r}_{\mathrm{ij}} \quad$ vector from pointing from atom $\mathrm{j}$ to $\mathrm{i}$

$r_{\mathrm{e}} \quad$ equimolar radius, $\AA$ 
$t \quad$ total simulation time, $\mathrm{s}$

$\mathbf{u}$ average velocity of total atoms in one control volume

$v \quad$ velocity, $\mathrm{m} / \mathrm{s}$

$v_{\mathrm{s}} \quad$ speed of sound, $\mathrm{m} / \mathrm{s}$

$\mathrm{V}_{\mathrm{i}} \quad$ velocity of atom $i^{\text {th }}$

$x \quad$ coordinate, $\mathrm{m}$

$A$ area, $\mathrm{m}^{2}$ (surface area expose to argon flow, Chapter 7)

C volumetric heat capacity

$D \quad$ characteristic length $(\AA)$

$E_{a b} \quad$ potential function, $\mathrm{J}$

E Young's modules, GPa

$E_{k e, e x} \quad$ exchanged kinetic energy between each pair, J

$K_{\mathrm{r}} \quad$ elasticity constant of harmonic bond, $\mathrm{eV} / \AA^{2}$

$K_{\theta} \quad$ elasticity constant of harmonic angle, $\mathrm{eV} / \mathrm{rad}^{2}$

$U_{a b} \quad$ potential function, $\mathrm{J}$

$L \quad$ length of bond, $\AA$

$M_{\mathrm{i}} \quad$ total mass of single molecule, $\mathrm{kg}$

M $\quad$ mass of liquid argon of $\mathrm{J}^{\text {th }}$ control volume, $\mathrm{kg}$

$N \quad$ number of atoms

$\mathrm{Nu} \quad$ Nusselt number

$P_{\text {mo,ex,i }}$ amount of exchanged momentum, kg-m/s 
$Q \quad$ Total energy passes through the copper wall

$T \quad$ temperature, $\mathrm{K}$

U velocity, $\mathrm{m} / \mathrm{s}$

$\mathbf{U}_{\mathrm{J}} \quad$ velocity of liquid argon of $\mathrm{J}^{\text {th }}$ control volume

$V \quad$ volume, $\mathrm{m}^{3}$ (potential energy, J, Chapter 7)

\section{Greek Symbols}

$\alpha \quad$ damping factor

$\delta \quad$ Dirac Delta function

$\delta t^{\mathrm{P}} \quad$ time-step in molecular dynamics simulation, $\mathrm{s}$

$\Delta t \quad$ time step, ps

$\varepsilon \quad$ depth of potential well, Kcal/mole

$\mu \quad$ viscosity, mPas

$\rho \quad$ density, $\mathrm{g} / \mathrm{cm}^{3}$

$\xi \quad$ intermediate variable

$v \quad$ viscosity, $\mathrm{m}^{2} / \mathrm{s}$

$\sigma \quad$ minimal distance between atoms when potential energy equal zero, $\AA$

$\theta \quad$ degree of $\mathrm{H}-\mathrm{O}-\mathrm{H}$ in water molecule, ${ }^{\circ}$ (degree of angle, ${ }^{\circ}$, chapter 5,6 )

$\varphi \quad$ degree of angle composed by oxygen and two artificial charged sites in five-site model, ${ }^{\circ}$

$\Theta \quad$ quadrupole moment tensor, $\mathrm{C} \mathrm{m}^{2}$

$\omega_{D} \quad$ Debye frequency, $\mathrm{s}^{-1}$ 


\section{Subscripts}

$\begin{array}{ll}\alpha & \mathrm{x}, \mathrm{y}, \mathrm{z} \\ \beta & \mathrm{x}, \mathrm{y}, \mathrm{z} \\ l & \text { liquid } \\ v & \text { vapor } \\ \text { B } & \text { H-O bond } \\ \text { C } & \text { coulomb related variable } \\ \text { CC } & \text { interaction between carbon atoms } \\ \text { EAM } & \text { embedded-atom method } \\ \text { H } & \text { hydrogen element } \\ \text { LJ } & \text { Lennard-Jones } \\ \text { L } & \text { artificial site in five site model } \\ \text { M } & \text { artificial site in four site model } \\ & \end{array}$




\section{CHAPTER 1 INTRODUCTION}

\subsection{Introduction}

Micro-/nano- technology has drawn a great attention in the past decades due to the desirability of miniaturization of devices. Many applications in various fields, such as biotechnology, high-performance computing, and microelectronic circuit, are increasingly evident. At the same time, many new issues are raised up due to the failure of application of conventional theory in thermal related mechanics design. For example, temperature prediction value from Fourier's Law is lower by one magnitude than experimental data [1]. Obviously, the product designed based conventional theory many cause instability problems for real application. In order to reveal unknown mechanism in small scale, numerical simulation approach to some extent has no alternative in comparison with experimental method that cost highly and also has problems with data measurement for extreme small scales(both in time and space), especially for non-equilibrium thermal process.

Generally speaking, there are mainly three modeling approaches existing for solving micro-scale problem, namely, continuum models, Boltzmann transport equation, and molecular approach.

For micro-scale heat transfer continuum models, they can be further separated into several categories, according to the basic transport mechanisms and the type of energy carriers involved. Most familiar Fourier's law usually will be adopted if the energy 
carrier undergoes frequent collision and transport is diffusive. However, the most serious theoretical problem of Fourier's law is that it implies an infinite speed of wave speed, in other words, it allows the entire system to feel the thermal effect when the surface of an object is instantaneously heated. To account for the finite speed of thermal wave, Catteneo's equation[2] which essentially introduced a relaxation time $\tau$ that represents the time required to develop the heat flux after a temperature gradient is applied is introduced. Further, Tzou[3] introduced dual-phase model that has another relaxation time which accounts for the time period required to establish temperature gradient when a flux is applied. Another manner of continuum model is establishing energy conservation equation for each energy carriers, and couple the energy transfer between different energy carriers with coupling terms, such as Two-Temperature model[4] (TTM). However, continuum modeling approach may suffer from failure for two reasons no matter how it is constructed. One is that state property of material, such as temperature, cannot be well defined when the interested domain approaches to mean free path of molecule, thereby the continuum heat transfer equations become meaningless; the other is the non-linearity of thermo-physical properties due to size effect, such as thermal conductivity can be reduced significantly due to increased defect and/or grain boundary scattering [5]. When continuum model method is not able to capture certain phenomena, a more common and slightly more difficult method based on Boltzmann transport equation is employed. Boltzmann transport equation (BTE) is a conservation equation that conserves the number of particles in the phase space, as shown in equation 1.1. 


$$
\begin{aligned}
& \frac{\partial}{\partial t}\left[f(x, \mathbf{P}, t) d V_{x} d V_{P}\right]+\mathbf{v} \cdot \nabla_{x}\left[f(x, \mathbf{P}, t) d V_{x} d V_{P}\right]+\mathbf{F} \cdot \nabla_{P}\left[f(x, \mathbf{P}, t) d V_{x} d V_{P}\right] \\
& =\left\{\frac{\partial}{\partial t}\left[f(x, \mathbf{P}, t) d V_{x} d V_{P}\right]\right\}_{\text {coll }}
\end{aligned}
$$

It has been proved that Fourier's Law and Wave equation can be both obtained if apply Boltzmann transport equation to energy carrier phonon. However, the great difficulty is raised up by the collision term which is on the right-hand side, whose collision nature has not been fully examined though general expressions for the collisional frequencies of electron-electron, electron-phonon, and phonon-phonon scattering have already been derived. When Boltzmann transport equation has difficulties to be solved, a more computational exhaustive approach, molecular dynamics simulation, have to be adopted. Molecular dynamics method is extensively developed recently due to recent advances in computational capabilities; mainly it includes lattice dynamic approach, molecular dynamics simulations, and Monte Carlo simulations. Lattice dynamics approach is effective in calculating phonon dispersion relations; molecular dynamics has been proved that it has well predicted many phenomena in micro-scale with appropriate potential function; Monte Carlo simulation is also adopted in many studies, especially those systems involve electron and phonon.

Since the work will be presented here is mainly based on classical molecular dynamics simulation, more detailed information on it will be provided in the following section. 


\subsection{Classical molecular dynamics}

Before we jump into the topic on how classical molecular dynamic is related to quantum mechanics, it worth to briefly review the history of current classical molecular dynamics framework. In 1957, Alder and Wainwright complete the first simulation on phase transition using MD simulation with hard sphere system[6]; In 1960, Gibson, Goland, Milgram and Vineyard[7], introduced the soft potential function for the first time into MD system that only have 500 atoms though; In 1964, Rahman[8] performed the first MD simulation with well-known Lennard-Jones(LJ) potential for argon and his work identified the modern MD simulation framework; In 1967, for the first time, Verlet [9]introduced the very famous and popular used Verlet time integration algorithm to MD simulation to study phase diagram of argon using LJ potential; In 1974, Stillinger and Rahman[10] conducted MD simulation on liquid water which is identified as the first MD simulation of a realistic system; In 1977, McCammon[11], et al. for the first time applied MD simulation to big molecule, protein. It is never too many of giving thanks to those great pioneers of classical molecular dynamics.

In fact, molecular dynamics is a quite wide topic even though it is only one branch of atomistic simulation method. It is well accepted that Schrödinger equation plays the role as Newton's equation does in classical mechanics. Ideally, any information of a dynamic system can be determined by solving Schrödinger equation. However, the equation is so complex that it can only be solved analytically for a very limited number of cases. Even the direct numerical simulation on computer can only provide solutions for very simple 
system that also has a few number of particles due to the high dimension of the space the Schrödinger equation involve. Take an example of nuclei and electron system, the timeindependent Schrödinger equation can be expressed as,

$$
\hat{H} \Psi\left(\mathbf{r}_{n} ; \mathbf{R}_{i}\right)=E \Psi\left(\mathbf{r}_{n} ; \mathbf{R}_{i}\right)
$$

where $\Psi\left(\mathbf{r}_{n} ; \mathbf{R}_{i}\right)$ is wave-function, $\mathbf{r}_{n}$ is position vector of electron, $\mathbf{R}_{i}$ is position vector of nuclei, $\mathrm{E}$ is total energy of the system and $\hat{H}$ is quantum operator which can be converted from the Hamiltonian expression[12],

$$
H=\sum_{i} \frac{\mathbf{P}_{i}^{2}}{2 M_{i}}+\sum_{n} \frac{\mathbf{p}_{n}^{2}}{2 m}+\frac{1}{2} \sum_{i j} \frac{Z_{i} Z_{j} e^{2}}{\left|\mathbf{R}_{i}-\mathbf{R}_{j}\right|}+\frac{1}{2} \sum_{n n^{\prime}} \frac{e^{2}}{\left|\mathbf{r}_{n}-\mathbf{r}_{n^{\prime}}\right|}-\sum_{i n} \frac{Z_{i} e}{\left|\mathbf{R}_{i}-\mathbf{r}_{n}\right|}
$$

where $\mathbf{P}_{i}$ and $\mathbf{p}_{n}$ represents momenta of nuclei and electron, $Z_{i}$ is the atomic number of nucleus; $M_{i}$ and $m$ are masses of nuclei and electron respectively. As mentioned above, very few and simple cases can be solved analytically or numerically. Due to the difficulties of solving the Schrödinger equation, in 1923, Born and Oppenheimer noted that the mass of nuclei is much heavier than electrons; thereby they realized that the nuclei move on at time scale which is about two orders of magnitude longer than that of the electrons. By applying this assumption, the Schrödinger equation can be further split into equations that govern the dynamical behavior of nuclei and electron separately as following,

$$
\left\{\begin{array}{c}
H_{e l} \Phi\left(\mathbf{r}_{n} ; \mathbf{R}_{i}\right)=V\left(\mathbf{R}_{i}\right) \Phi\left(\mathbf{r}_{n} ; \mathbf{R}_{i}\right) \\
H_{n u} \Xi\left(\mathbf{R}_{i}\right)=E \Xi\left(\mathbf{R}_{i}\right)
\end{array}\right.
$$

where 


$$
H_{e l}=\sum_{n} \frac{p_{n}^{2}}{2 m}+\frac{1}{2} \sum_{i j} \frac{Z_{i} Z_{j} e^{2}}{\left|\mathbf{R}_{i}-\mathbf{R}_{j}\right|}+\frac{1}{2} \sum_{n n^{\prime}} \frac{e^{2}}{\left|\mathbf{r}_{n}-\mathbf{r}_{n^{\prime}}\right|}-\sum_{i n} \frac{Z_{i} e}{\left|\mathbf{R}_{i}-\mathbf{r}_{n}\right|}
$$

and

$$
H_{n u}=\sum_{i} \frac{P_{i}^{2}}{2 M_{i}}+V\left(\mathbf{R}_{i}\right)
$$

The classical molecular dynamics is totally based on solving equation 1.6, and all the electron effects are incorporated in potential function $V\left(\mathbf{R}_{i}\right)$. All the terminologies of molecular dynamic in the following section are referring to classical molecular dynamics if there is no special notification. In practice, the system is driven by solving a set of Newton's equation that drives the evolution of the system by employing different efficient algorithms, such Verlet algorithm, Predictor-corrector, Leap-frog algorithm, Beeman's algorithm etc.

As mentioned above, classical molecular dynamic is a wide topic, this method can be further categorized into equilibrium molecular dynamics (EMD) and non-equilibrium molecular dynamics (NEMD) according to the state simulation system. Both of them will be briefly introduced in the following sections.

\subsubsection{Equilibrium molecular dynamics}

EMD is statistical mechanics theory based method. In order to have a better understanding of EMD, it is necessary to introduce very important concept of ensemble. An ensemble is a collection of all possible system which has different microscopic state but have an identical macroscopic or thermodynamic state. For example, a collection of 
all possible configuration $(\mathbf{p}, \mathbf{q})$ that has fixed number of atoms $(\mathrm{N})$, fixed volume $(\mathrm{V})$ and fixed energy (E) is an ensemble (NVE), where $\mathbf{p}$ represent momentum vector and $\mathbf{q}$ is position vector of the system. Any interested property of the molecular system can be obtained by averaging all possible property values within the ensemble. For example, let $\mathrm{A}(\mathbf{p}, \mathbf{q})$ represent a property with $\mathbf{p}$ and $\mathbf{q}$ configuration, then the property in macroscopic can be estimated by integrating over physical and momentum space, as shown in equation $(1.7)$

$$
\langle A\rangle_{\text {ensemble }}=\iint d \mathbf{p} d \mathbf{q} A(\mathbf{p}, \mathbf{q}) \rho(\mathbf{p}, \mathbf{q})
$$

where $\rho(\mathbf{p}, \mathbf{q})$ is phase point probability density function. However, due to difficulties in very high dimensional integration and impossibility in traverse all phase points; it is not realistic to perform molecular dynamics by following the concept introduced above. Alternatively, an ergodic hypothesis which state ensemble average equals to time average if the number of samples is sufficiently enough is employed.

In addition, much other useful formalism under the framework of equilibrium molecular dynamics is developed, like Green-Kubo formulas which relate the ensemble average of the auto-correlation of the flux.

\subsubsection{Non-equilibrium molecular dynamics simulation}

NEMD, which is similar to EMD, is also based on time-reversible equations of motion, Newton's equation. A possible view point to distinguish NEMD from EMD is Liouville equations, as expressed by equation 1.8. 


$$
\frac{d \rho}{d t}=\frac{\partial \rho}{\partial t}+\sum_{i=1}^{n}\left(\frac{\partial \rho}{\partial q_{i}} \dot{q}_{i}+\frac{\partial \rho}{\partial p_{i}} \dot{p}_{i}\right)
$$

where $\rho=\rho(\mathbf{q}, \mathbf{p}, t)$ is phase density function which describe the distribution of phase points. If $\frac{\partial \rho}{\partial t}=0$, then it is identified as EMD, whereas it is NEMD. In comparison with equilibrium molecular dynamic, non-equilibrium molecular dynamics not only provide another mean of computing transport coefficient, but also improve the calculation efficiency. Time correlation function, which is commonly used in EMD to compute transport properties based on Green-Kubo equation, like viscosity and thermal conductivity, require a large number of statistical samples and also sensitive to the sample intervals. Moreover, significant statistical error cannot be avoided because they represent the average response to naturally occurring fluctuation which is relatively smaller. Intuitively, NEMD also provide a degree of freedom to exam the system response to a larger perturbation.

Generally, non-equilibrium molecular dynamics can be categorized into two types according to the ways used to affect the system, either through boundary or internal atoms. For the former, using non-period boundary condition that exposes the atoms to external momentum for the molecular box, like evaporation study of liquid; for the later, introducing the perturbation through equation of motion by modifying dynamics system, like introduce instant heat to system partially by interrupting the equations. The work in this thesis are mainly done with NEMD, different techniques will be shown in the flowing chapters, especially, Chapter 2, 3 and 4. 


\subsection{Water Models}

Since the work presented in the thesis is mainly based liquid water, it will be quite helpful for current and future research to review water models existing in the literature.

In spite of simplicity of water molecule, there still no universally applicable water model available in literature to reproduce all properties successfully. To my understanding, this mainly caused by at least two reasons. The first one is lack of effective potential to describe interaction between water molecules. In fact, water potential is mainly composed of short-range part, van der Waals potential, and long range part, coulombs potential. The current potential being popularly employed are mainly twobody potentials, however, it is shown that three-body effects contribute $14.5 \%$ to the internal energy for single water molecule[13]. The second reason could be the inaccuracy description of electron contribution. For example, many water models have emphasized the molecular polarization effect which is caused by many reasons, such as the redistribution of electrons or changes in the bond lengths and angles or the rotation of the whole molecule, on various macroscopic behaviors. Due to those difficulties, many models now built are most likely constructed for a specific purpose usage.

According to decent literature review on water model by Martin Chaplin[14], water models can be separated into four categories, namely as type a, b, c and d in terms of structure, as shown in Figure 1-1. All $a, b$ and $c$ types are planar model, whereas type $d$ is three dimensional. Among them, type a is also named as three site water model, type b and c are called as four-site water model, and type d is called five-site model. Error! 
eference source not found. shows 23 popular rigid water models along with their structures and associated parameters. If models enable the bond stretching and angle bending with certain manners, such as harmonic spring, there are 46 distinct models existing in the literature. In fact, some the parameters, like $\sigma$ and $\varepsilon, l_{1}, l_{2}$, will be slightly changed if different solving scheme are applied to coulomb potential. For example, like $\sigma$ and $\varepsilon$ should be modified to be other values if $\mathrm{P}^{3} \mathrm{M}$ solver is used to solve for long-range force. Therefore, it is clearly to see that more than 46 water models available in the literature in total.

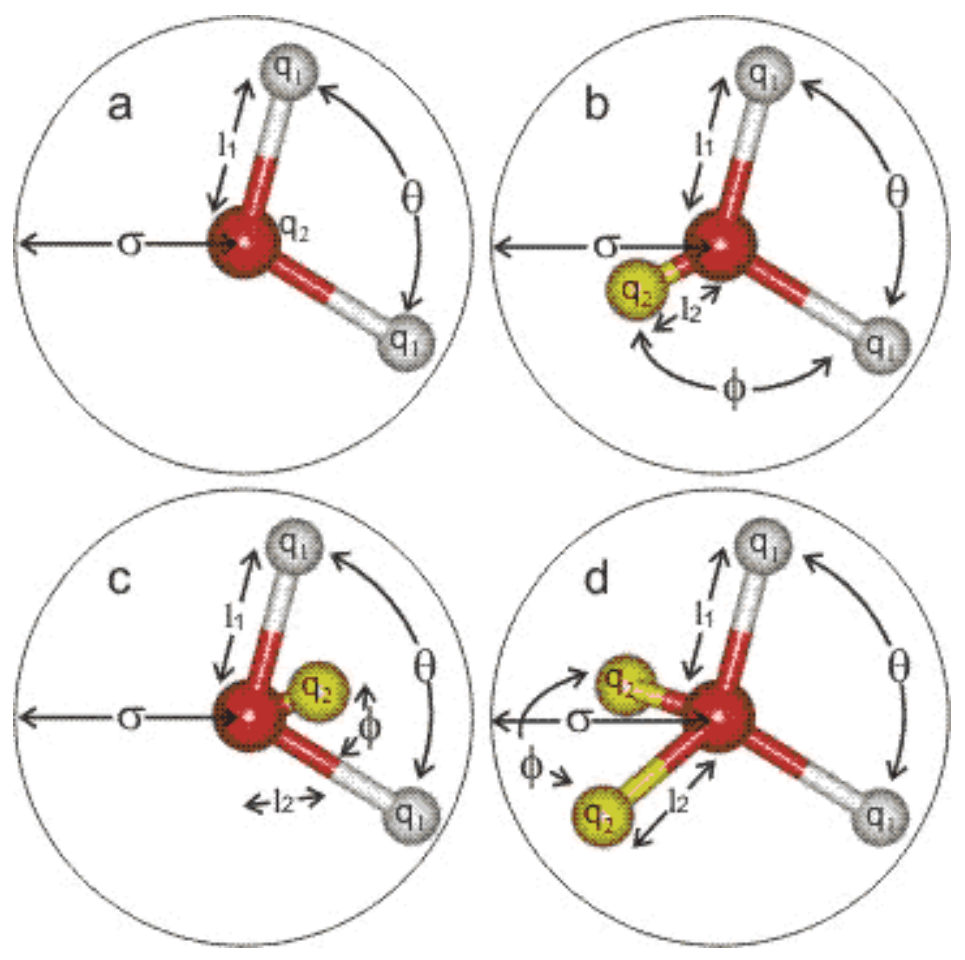

Figure 1-1 Four types of water molecules[14] 
Table 1-1 Parameters for different types of water models[14]

\begin{tabular}{|c|c|c|c|c|c|c|c|c|c|}
\hline Model & Type & $\sigma(\AA)$ & $\varepsilon \mathrm{kJ}$ mol-1 & $11(\AA)$ & $\mathbf{l}_{2}(\AA)$ & $\mathbf{q}_{1}(\mathbf{e})$ & $\mathbf{q}_{2}(\mathbf{e})$ & $\theta\left({ }^{\circ} \mathrm{C}\right)$ & $\varphi\left({ }^{\circ} \mathrm{C}\right)$ \\
\hline SSD & -8 & 3.016 & 15.319 & - & - & - & - & 109.47 & 109.47 \\
\hline SPC & a & 3.166 & 0.65 & 1 & - & 0.41 & -0.82 & 109.47 & - \\
\hline SPC/E & a & 3.166 & 0.65 & 1 & - & 0.4238 & -0.8476 & 109.47 & - \\
\hline SPC/HW(D2O) & a & 3.166 & 0.65 & 1 & - & 0.435 & -0.87 & 109.47 & - \\
\hline $\mathrm{SPC} / \mathrm{Fw}$ & $\mathrm{a}$ & 3.166 & 0.65 & 1.012 & - & 0.41 & -0.82 & 113.24 & - \\
\hline TIP3P & $\mathrm{a}$ & 3.15061 & 0.6364 & 0.9572 & - & 0.417 & -0.834 & 104.52 & - \\
\hline TIP3P/Fw & a & 3.1506 & 0.6368 & 0.96 & - & 0.417 & -0.834 & 104.5 & - \\
\hline PPC & $\mathrm{b}$ & 3.234 & 0.6 & 0.943 & 0.06 & 0.517 & -1.034 & 106 & 127 \\
\hline TIP4P & $\mathrm{c}$ & 3.15365 & 0.648 & 0.9572 & 0.15 & 0.52 & -1.04 & 104.52 & 52.26 \\
\hline TIP4P-Ew & $\mathrm{c}$ & 3.16435 & 0.680946 & 0.9572 & 0.125 & 0.52422 & -1.04844 & 104.52 & 52.26 \\
\hline TIP4P-FQ & $\mathrm{c}$ & 3.15365 & 0.648 & 0.9572 & 0.15 & 0.631 & -1.261 & 104.52 & 52.26 \\
\hline TIP4P/Ice & $\mathrm{c}$ & 3.1668 & 0.8822 & 0.9572 & 0.1577 & 0.5897 & -1.1794 & 104.52 & 52.26 \\
\hline TIP4P/2005 & $\mathrm{c}$ & 3.1589 & 0.7749 & 0.9572 & 0.1546 & 0.5564 & -1.1128 & 104.52 & 52.26 \\
\hline SWFLEX-AI & $\mathrm{c}$ & \multicolumn{2}{|c|}{ Four terms used } & 0.9681 & $0.141,3$ & 0.6213 & -1.2459 & 102.71 & 51.351 \\
\hline $\mathrm{COS} / \mathrm{G} 3$ & $\mathrm{c}$ & 3.17459 & 0.9445 & 1 & 0.15 & 0.450672 & -0.901344 & 109.47 & - \\
\hline GCPM & $\mathrm{c}$ & 3.69 & 0.91464 & 0.9572 & 0.27 & 0.6113 & -1.2226 & 104.52 & 52.26 \\
\hline SWM4-NDP & $\mathrm{c}$ & 3.18395 & 0.88257 & 0.9572 & 0.24034 & 0.55733 & -1.11466 & 104.52 & 52.26 \\
\hline $\mathrm{ST} 2$ & $\mathrm{~d}$ & 3.1 & 0.31694 & 1 & 0.8 & 0.24357 & -0.24357 & 109.47 & 109.47 \\
\hline TIP5P & $\mathrm{d}$ & 3.12 & 0.6694 & 0.9572 & 0.7 & 0.241 & -0.241 & 104.52 & 109.47 \\
\hline TIP5P-Ew & d & 3.097 & 0.7448 & 0.9572 & 0.7 & 0.241 & -0.241 & 104.52 & 109.47 \\
\hline TTM2-F & $\mathrm{c}$ & \multicolumn{2}{|c|}{ Five parameters used } & 0.9572 & 0.7 & 0.574 & -1.148 & 104.52 & 52.26 \\
\hline POL5/TZ & d & 2.98374 & 4 & 0.9572 & 0.5 & varies5 & -0.42188 & 104.52 & 109.47 \\
\hline Six-site & $\mathrm{c} / \mathrm{d}$ & $\begin{array}{l}3.115 \mathrm{OO} \\
0.673 \mathrm{HH} \\
\end{array}$ & $\begin{array}{c}0.715 \mathrm{OO} \\
0.115 \mathrm{HH} \\
\end{array}$ & 0.98 & $\begin{array}{c}0.8892 \mathrm{~L} \\
0.230 \mathrm{M} \\
\end{array}$ & 0.477 & $\begin{array}{c}-0.044 \mathrm{~L} \\
-0.866 \mathrm{M} \\
\end{array}$ & 108 & 111 \\
\hline
\end{tabular}


Table 1-2 gives some published results on dipole moments, dielectric constant, selfdiffusion, average configurational energy, maximum density, and expansion coefficient, which are done by many other researchers, with various water models. It is clearly shown that different water models have very different strength in predicting those properties. Almost all the models has good performance in predicting dipole moment, average configuration energy, while for the other properties a big variation among them can be observed, for example, maximum density. It is worth to notice that model PPC which is a simple three-site water model has an impressive performance in almost all of the properties prediction; however, its structure is very different from what it is in minds, which has a symmetric structure. Though many works have been well done and reported, some properties that related to liquid water flow's dynamic property or thermal property, which are important for thermal flow related engineering problems, have not been examined, for example, shear viscosity and thermal conductivity. In this work, eight rigid water models are selected to study their performances in predicting those properties in Chapter 2 and Chapter 3. Chapter 2 shows thermal conductivity, shear Viscosity and specific Heat of rigid water models; Chapter 3 studied the temperature-dependent thermal conductivity and shear viscosity for rigid water models; Chapter 4 investigated nanobubble's growth and annihilation in liquid water by non-equilibrium molecular dynamics; Chapter 5 reveals contact angle of water droplet on a single wall carbon nanotube plate; Chapter 6 studied the behavior of a thin water film on a hot copper plate; Chapter 7 provide an atomistic-continuum hybrid simulation framework based CFDEM that 
combined massive particle simulator LIGGGHTs and CFD toolbox OpenFOAM, and gives an insight of convective phenomena in micro-scale for argon fluid flow on copper wall. 
Table 1-2 properties predicted by different water models[14]

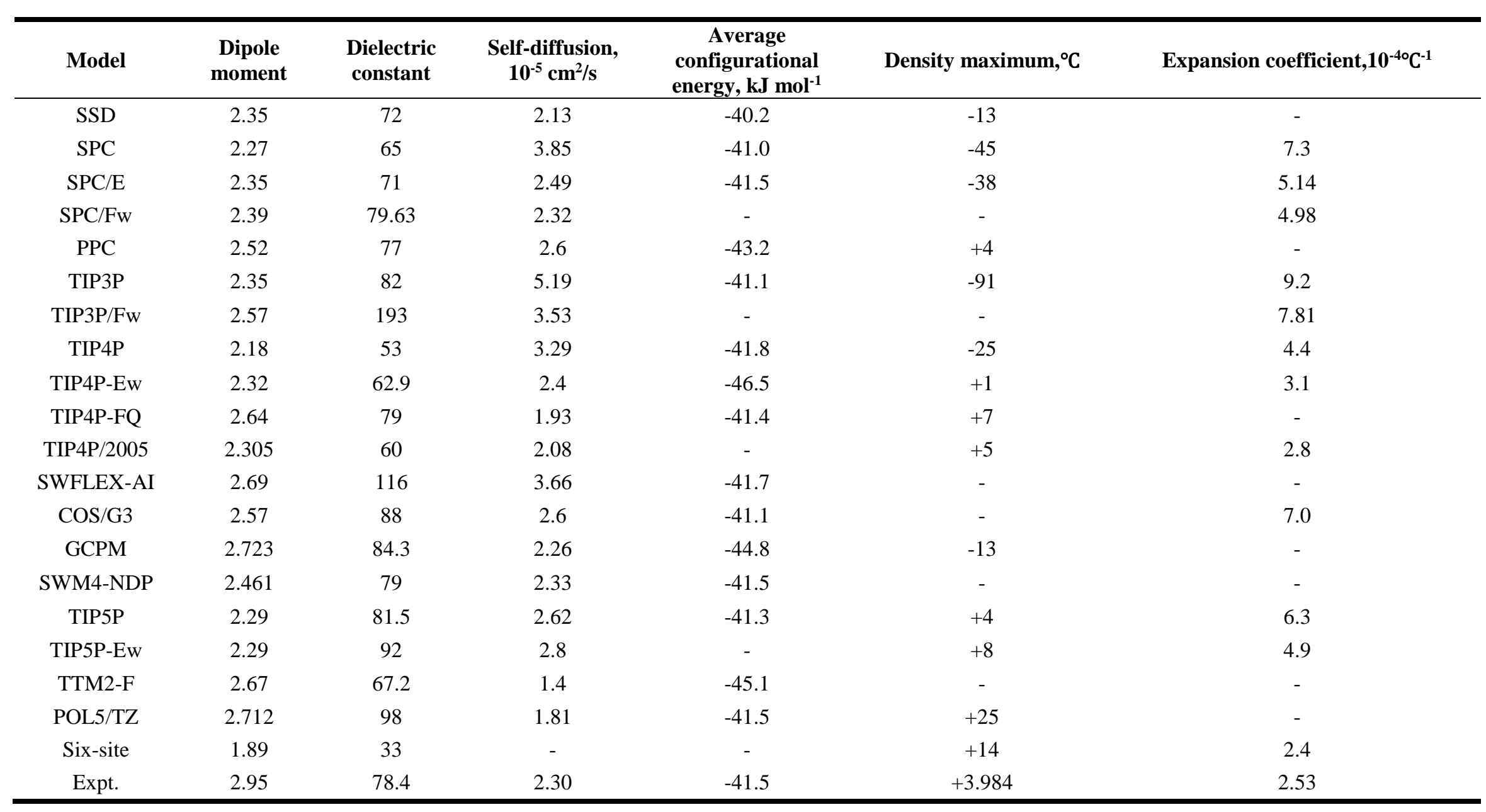




\subsection{Open-Source}

\subsubsection{LAMMPS}

LAMMPS with an acronym for Large-scale Atomic/Molecular Massively Parallel Simulator is distributed as an open source classical molecular dynamics code under the terms of the GPL, by Sandia National Laboratories. It has potentials for soft materials (biomolecules, polymers) and solid-state materials (metals, semiconductors) and coarsegrained or mesoscopic systems. It can be used to model atoms or, more generically, as a parallel particle simulator at the atomic, meso, or continuum scale. It runs on single processors or in parallel using message-passing techniques and a spatial-decomposition of the simulation domain. The code is designed to be easy to modify or extend with new functionality.

\subsubsection{OpenFOAM}

Introduction from OpenFOAM webpage: The OpenFOAM® (Open Field Operation and Manipulation) CFD Toolbox is a free, open source CFD software package produced by OpenCFD Ltd. It has a large user base across most areas of engineering and science, from both commercial and academic organizations. OpenFOAM has an extensive range of features to solve anything from complex fluid flows involving chemical reactions, turbulence and heat transfer, to solid dynamics and electromagnetics. It includes tools for meshing, notably snappyHexMesh, a parallelised mesher for complex CAD geometries, and for pre- and post-processing. Almost everything (including meshing, and pre- and 
post-processing) runs in parallel as standard, enabling users to take full advantage of computer hardware at their disposal.

By being open, OpenFOAM offers users complete freedom to customise and extend its existing functionality, either by themselves or through support from OpenCFD. It follows a highly modular code design in which collections of functionality (e.g. numerical methods, meshing, physical models, ...) are each compiled into their own shared library. Executable applications are then created that are simply linked to the library functionality. OpenFOAM includes over 80 solver applications that simulate specific problems in engineering mechanics and over 170 utility applications that perform preand post-processing tasks, e.g. meshing, data visualization, etc.

\subsubsection{CFDEM}

CFDEM is mainly an extension of library based on OpenFOAM source code, by integrating LIGGGHTs, which is an improved version of LAMMPS, into an innovative library. Users are allowed to create solver that is able to couple particle based approach, granular particles, and continuum method, Navier-Stokes equation.

\subsection{Dissertation Objectives}

The objective of this work is outlined as:

1 Investigate water properties, shear viscosity, thermal conductivity, and specific heat, through non-equilibrium molecular dynamics, by using eight popular water models. Not only the method developed here is able to directly apply to other water models, but also it will show the strengths of those selected rigid models. 
2 Study on dynamic behavior, such as nano-bubble growth/annihilation, rapid boiling of thin water film, and contact behavior on hydraulic material, is also be done to reveal the special dynamic and thermal phenomena occur in the 'smallWorld'.

3 With the purpose of making full use of the existing high-performance open source codes, LIGGGHTs and OpenFOAM, a dynamic library which is linkable during computation is developed, and a solver that is able to coupling MD and CFD is developed, validated and applied to investigate heat transfer behavior between solid copper wall and liquid argon flow. 


\section{Chapter 2 Thermal Conductivity, Shear Viscosity and Specific Heat of Rigid Water Models}

\subsection{Introduction}

In the past decades, many water models are developed along with the intensive investigation on water by large number of molecular dynamics or Monte Carlo simulations. A 2002 review indicates that there are 46 water models[15], which were classified as rigid, flexible and polarizable models[16]. Recently, researchers proposed new well-performance models which are reparameterized based on the existing ones. For instance, TIP4P/2005[17], which is designed to be a general purpose model for condensed phase of water, has an impressive performance in predicting a variety number of thermophysical properties; TIP4P-Ew[18], which is another extend version of four-site rigid water model, has a global improvement of predicting water properties; It has been reported that TIP5P-Ew[19] has great performance in reproducing experiment data for liquid water.

However, none of the above models has the capability to perfectly reproduce all properties of water, which probably leads to confusion when researchers need to choose appropriate model in the molecular dynamics simulations of different problems. For example, different water solvent in biology molecular dynamics can lead to reasonable or unreasonable results[20]. Furthermore, although various properties are investigated and advantage and disadvantage also are reported, independent papers that report the comparison the performances on reproducing thermophysical properties from different models are seldom. González and Abascal calculated the shear viscosity with Green- 
Kubo model and compared the results obtained from five rigid water models, which include TIP3P[21], TIP4P[21], TIP5P[22], SPC/E[23] and TIP4P/2005, with experiment data[24]. However, evaluations of different models on predicting thermal conductivity and specific heat have not been done. There exist two main methods, equilibrium molecular dynamics (EMD) and nonequilibrium molecular dynamics (NEMD), to determine shear viscosity and thermal conductivity. Green-Kubo method[25] is based on EMD that transport properties are related to the time integral of a correlation function. It usually can predict the result with a reasonable accuracy and precision. However, it suffers from the difficulties on complications of determining the microscopic heat flux (or other required instant properties) and slow convergence of the time integral of the heat flux (or other required instant properties) autocorrelation function. To overcome these limits of Green-Kubo method, a non-equilibrium molecular dynamics approach has been developed by Florian Müller-Plathe[26, 27]. An accurate and efficient Reverse NonEquilibrium Molecular Dynamics (RNEMD) method for monoatomic molecular fluids was proposed. Later, Bedrov and Smith[28] extended the RNEMD to rigid polyatomic molecular fluids while still conserve energy and momentum of the system. However, results obtained from RNEMD somehow depend on swap frequency and carefully choosing the value is required.

In this paper, thermal conductivity will be calculated using RNEMD method for liquid water under one atmosphere and different temperatures (298K, 318K). The reason of choosing RNEMD is that the operations of this approach is similar to real technique when measuring thermal or dynamic properties of fluids in the laboratories; thus it can physically reflect the properties of fluids in a "real" situation. However, it should also be 
pointed out that the fluxes imposed in these MD simulations are different compared to real experiments, so the convergence of the calculated results with respect to the imposed fluxes should be checked. For RNEMD, the result must be converged as long as a steady state is established in the simulation box. For shear viscosities, a similar approach ${ }^{[15]}$ is used to impose momentum flux $\left(j_{z}\right)$ to the system by exchanging momentum of a mount of molecules in two specific slabs and then calculate the stable velocity gradient in a selected direction. Heat capacities will be calculated according to the assumed linear response[29] between enthalpy (or internal energy) and temperature. Therefore, enthalpy and internal energy of the system will be recorded during a gradual cooling process of the system. The following eight rigid models, SPC [30, 31], SPC/E, TIP3P, TIP4P, TIP4P/2005, TIP4P-Ew, TIP5P and TIP5-Ew, are selected to carry out these simulations in this work based on their relatively successful performance as reported in the literatures.

\subsection{Physical Model and Method}

For classical molecular dynamics, potential function plays a key role in driving atoms' evolutions in space. All potential functions of rigid water molecules share the same form, which consist of the contributions from electrostatic, dispersion and repulsive forces:

$$
E_{a b}=\sum_{i}^{\text {on a on } b} \sum_{j} \frac{k_{C} q_{a_{i}} q_{b_{j}}}{r_{a_{i} b_{j}}}+\sum_{i}^{\text {on a on } b} \sum_{j} 4 \varepsilon_{a_{i} b_{j}}\left[\left(\frac{\sigma_{a_{i} b_{j}}}{r_{a_{i} b_{j}}}\right)^{12}-\left(\frac{\sigma_{a_{i} b_{j}}}{r_{a_{i} b_{j}}}\right)^{6}\right]
$$

where, $a$ and $b$ denotes two different molecules, subscript $i$ and $j$ represent atom $i$ in one individual molecule for all three-site rigid water molecule, $k_{\mathrm{c}}$ is electrostatic constant. For four- and five-site rigid water models, $i$ and $j$ represent massless but charged site in the first summation, still denotes atom in the second summation. Short range force is 
neglected when the distance of two atoms exceeds the cutoff distance, while long range force is evaluated with PPPM method[32] which splits long range effect into short range and long range parts. For four- and five-site models, Coulomb forces on virtual sites are redistributed to real atoms[30]. All parameters for different potentials are given in Table $2-1$.

For rigid polyatomic molecule, the degree of freedom of each atom should be carefully treated through equation below when calculating the atomic temperature,

$$
\left(\frac{3-c}{2}\right) k_{B} T=\frac{1}{2} m v^{2}
$$

where $c$ is the number of constrains due to geometry constrain algorithm (here SHAKE ${ }^{[33]}$ is employed), and $k_{b}$ is Boltzmann constant. Since the shapes of all water molecules are geometrically fixed, $c$ can be calculated easily for each atom. Here $c$ is 1 if the degree of freedom counting method in reference[27] is applied, i.e., one constraint of one atom contribute to $1 / 2$ constrain. The temperature in each slab can be estimated by averaging all atomic temperature within, while heat flux $(q)$ can be calculated by accumulating exchanged molecular kinetic energies. After heat flux $(q)$ and temperature gradient are obtained, Fourier's Law can be employed to calculate thermal conductivity.

$$
k=-q / \nabla T
$$

For shear viscosity calculation, degree of freedom estimation will not be a concern, and all procedures are similar. Velocity in each slab is calculated locally by averaging all atoms' velocity[34]. Simulation is done until symmetric temperature or velocity profile is stably established. 
The enthalpy of the system, which will be used for specific heat calculation, is calculated by summing up internal energy and the product of pressure and volume of the system, while internal energy accounts for potential energy (Van der Waals, Coulomb pairwise energy, etc) and kinetic energy.

$$
H=E_{e}+P V=K_{e}+P_{e}+P V
$$

where $P$ and $V$ are pressure and the volume of the simulation box, $K_{e}$ is kinetic energy, $P_{e}$ is potential energy, and $E_{e}$ is internal energy. Specific heats at constant pressure and constant volume will be calculated through the following formulas:

$$
c_{p}=\left.\frac{1}{M}\left(\frac{\partial H}{\partial T}\right)\right|_{p}
$$

and

$$
c_{v}=\left.\frac{1}{M}\left(\frac{\partial E_{e}}{\partial T}\right)\right|_{v}
$$

where, $\mathrm{M}$ is the total mass of the simulation box.

In these simulations, all water molecules systems are treated in the same manner, except applying different potential and geometrical parameters (see Table 2-1). 
Table 2-1 Parameters of 8 rigid water models

\begin{tabular}{|c|c|c|c|c|c|c|c|c|}
\hline Model & $\sigma(\AA)$ & $\begin{array}{c}\varepsilon(\text { Kcal mole } \\
1)\end{array}$ & qH(e) & $q O(e)$ & $\theta^{\circ}$ & $\varphi^{\circ}$ & $\mathbf{q M} / \mathbf{q} \mathbf{L}(\AA))$ & $\mathbf{L B}(\AA ̊)$ \\
\hline SPC & 3.16600 & 0.15535 & 0.41000 & $\begin{array}{c}- \\
0.82000\end{array}$ & 109.47 & $\mathrm{~N} / \mathrm{A}$ & $\mathrm{N} / \mathrm{A}$ & 1.00000 \\
\hline SPC/E & 3.16600 & 0.15535 & 0.42380 & $\begin{array}{c}0.84760 \\
-\end{array}$ & 109.47 & $\mathrm{~N} / \mathrm{A}$ & $\mathrm{N} / \mathrm{A}$ & 1.00000 \\
\hline TIP3P & 3.15061 & 0.15210 & 0.41700 & $\begin{array}{c}0.83400 \\
-\end{array}$ & 104.52 & $\mathrm{~N} / \mathrm{A}$ & $\mathrm{N} / \mathrm{A}$ & 0.97520 \\
\hline TIP4P & 3.15365 & 0.16348 & 0.52000 & $\begin{array}{c}1.04000 \\
-\end{array}$ & 104.52 & $\mathrm{~N} / \mathrm{A}$ & 0.15000 & 0.97520 \\
\hline TIP4P-Ew & 3.16435 & 0.16275 & 0.52422 & 1.04844 & 104.52 & $\mathrm{~N} / \mathrm{A}$ & 0.12500 & 0.97520 \\
\hline TIP4P/2005 & 3.15890 & 0.18521 & 0.55640 & -1.11280 & 104.52 & N/A & 0.15460 & 0.97520 \\
\hline TIP5P & 3.12000 & 0.15999 & 0.24100 & $\begin{array}{c}0.24100 \\
-\end{array}$ & 104.52 & 109.47 & 0.70000 & 0.97520 \\
\hline TIP5P-Ew & 3.09700 & 0.17801 & 0.24100 & 0.24100 & 104.52 & 109.47 & 0.70000 & 0.97520 \\
\hline
\end{tabular}


For transport properties evaluation, all the simulation has been performed in canonical ensemble $(N V T)$ using the artificial mass based Nośe-Hoover thermostat[35] for a sample size of 900 water molecules cubic system, whose boundaries is treated as periodic with dimension of $30 \AA \times 30 \AA \times 30 \AA$. Thus the density is $996.67 \mathrm{~kg} / \mathrm{m}^{3}$. The simulation box is divided into 20 slabs in z direction, and 10 hottest molecules at bottom slab and coldest in middle slab will be selected to exchange kinetic energy (or momentum in the $x$-direction for shear viscosity calculation). The simulation time step is $1 \mathrm{fs}$ and the total time is 2 ns, excluding the equilibration stage. The cut-off distance for short range force is $1.04 \mathrm{~nm}$. An equilibration of $0.2 \mathrm{~ns}$ is carried out before RNEMD is performed. In order to achieve a reasonable linear response, swap frequencies of 200 and 50 are carefully chosen for thermal conductivity and shear viscosity calculations, respectively. The sample times of calculating gradients are 200 ps. For specific heat at constant volume simulation is performed in $N V T$ ensemble, while specific heat at constant pressure is done in NPT ensemble. Nośe-Hoover thermostat relax the temperature every 10 fs, while for Nośe-Hoover barostat control pressure every 100 fs. The value of internal energy in NVT ensemble and enthalpy in NPT ensemble are recorded when the temperature of the system is controlled at various temperatures namely, $283 \mathrm{~K}, 293 \mathrm{~K}$, $303 \mathrm{~K}, 313 \mathrm{~K}, 323 \mathrm{~K}, 333 \mathrm{~K}$ and $343 \mathrm{~K}$. In order to carry out simulation within the framework of the open-source molecular dynamics software LAMMPS[36], RNEMD for thermal conductivity and shear viscosity for polyatomic molecule fluid are implemented. While five-site rigid water models are also available with PPPM/TIP5P solver to obtain long range coulomb force, some additional function including counting degree of freedom for single atom are also added into the original codes. 
It is worth to notice that though TIP4P-Ew and TIP5P-Ew are designed to model with Ewald summation technique, since PPPM has can well approach the same precision of Ewald summation when mesh grids increased, and considering of it is high efficiency, all these long range coulomb interaction are solved with PPPM method. In order to get a reasonable mesh number for PPPM solver, shear viscosity, thermal conductivity, and specific heats are calculated with both standard Ewald summation and PPPM solver. The relative errors for each value are listed in Table 2-2, when mesh grids number is 30 in each direction. The results indicate that $30 \times 30 \times 30$ is sufficient to approximate the precision of standard Ewald summation.

Table 2-2 Comparison of results from different Kspace Solver

\begin{tabular}{cccc}
\hline $298 \mathrm{~K} / \mathrm{SPC}$ & Ewald & PPPM & $\mathrm{RE}$ \\
\hline $\mathrm{K}\left(\mathrm{W} \mathrm{m}{ }^{-1} \mathrm{~K}^{-1}\right)$ & 0.89 & 0.88 & $2.01 \%$ \\
$\eta(\mathrm{Pa} \cdot \mathrm{s})$ & $2.81 \times 10^{-5}$ & $2.72 \times 10^{-4}$ & $3.32 \%$ \\
$\mathrm{c}_{\mathrm{v}}\left(\mathrm{kJ} \mathrm{Kg}^{-1} \mathrm{~K}^{-1}\right)$ & 3.82 & 3.45 & $9.54 \%$ \\
$\mathrm{c}_{\mathrm{p}}\left(\mathrm{kJ} \mathrm{Kg}^{-1} \mathrm{~K}^{-1}\right)$ & 4.33 & 4.45 & $-2.69 \%$ \\
\hline
\end{tabular}

\subsection{Results and Discussion}

Figure 2-1, as a sample of generated temperature gradient, shows a linear temperature profile established at domain range from the top slab to the middle in TIP5P-Ew molecule composed system at $318 \mathrm{~K}$. The linear response is very good. The thermal conductivities at $298 \mathrm{~K}$ and $318 \mathrm{~K}$ are given in Table $2-3$. 


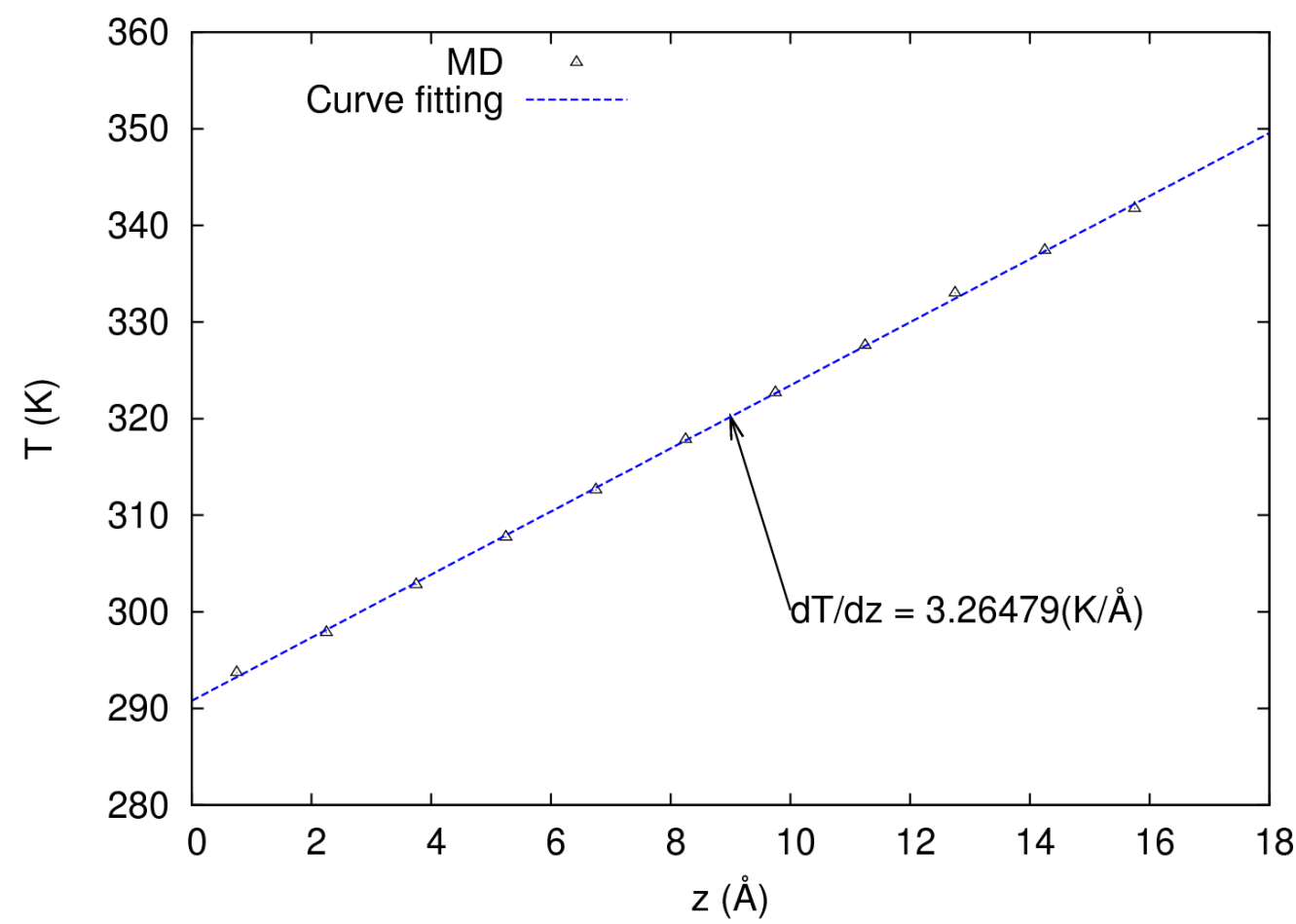

Figure 2-1 Established temperature gradient for TIP5P-Ew water system at 318K

It can be seen that both five-site rigid model have the better performance among all the reported cases: the relative errors at $298 \mathrm{~K}$ and $318 \mathrm{~K}$ are $11.32 \%$ and $4.08 \%$ (TIP5P), $1.87 \%$ and $7.59 \%$ (TIIP5P-Ew), respectively. For 298K, TIP5P-Ew has better performance than TIP5P model, while TIP5P performs better than TIP5P-Ew at 318K. In contrast, SPC model overestimate the thermal conductivity by $44.39 \%$ and $36.96 \%$; SPC/E has over predicted by $53.17 \%$ and $47.53 \%$; TIP3P has relative error at $45.59 \%$ and $49.08 \%$. Four-site models also yield large relative error range from $34.87 \%$ to $49.11 \%$. In addition, the experiment data shows the trends that thermal conductivity for liquid water increase with increasing temperature at one atmosphere pressure. The results in this work indicate that five models (SPC/E, TIP3P, TIP4P, TIP4P-Ew, TIP5P-Ew) could predict this trends. Therefore, take the trends prediction factor,TIP5P-Ew still can be considered to be the best. 
Table 2-3 Thermal conductivities at $298 \mathrm{~K}$ and $318 \mathrm{~K}$

\begin{tabular}{|c|c|c|c|c|}
\hline \multicolumn{5}{|c|}{$298 K$} \\
\hline & $\begin{array}{c}\text { heat flux } \\
\left(\begin{array}{c}\text { Kcal mole } \\
\left.{ }^{2} \text { fs }^{-1}\right)\end{array}\right. \\
\end{array}$ & $\begin{array}{c}\text { temperature } \\
\text { gradient } \\
\left(\mathrm{K} \AA^{-1}\right) \\
\end{array}$ & $\begin{array}{c}\text { thermal } \\
\text { conductivity } \\
\left(\mathrm{W} \mathrm{m}^{-1} \mathrm{~K}^{-1}\right) \\
\end{array}$ & $\begin{array}{c}\text { relative } \\
\text { error } \\
\%\end{array}$ \\
\hline SPC & $6.54 \times 10^{-5}$ & 2.59 & $0.880 \pm 0.020$ & 44.39 \\
\hline SPC/E & $6.28 \times 10^{-5}$ & 2.34 & $0.930 \pm 0.016$ & 53.17 \\
\hline TIP3P & $6.39 \times 10^{-5}$ & 2.51 & $0.880 \pm 0.019$ & 45.59 \\
\hline TIP4P & $6.33 \times 10^{-5}$ & 2.68 & $0.820 \pm 0.015$ & 35.02 \\
\hline TIP4P-Ew & $6.26 \times 10^{-5}$ & 2.43 & $0.900 \pm 0.013$ & 47.48 \\
\hline TIP4P-2005 & $6.33 \times 10^{-5}$ & 2.43 & $0.910 \pm 0.014$ & 49.11 \\
\hline TIP5P & $6.07 \times 10^{-5}$ & 3.12 & $0.680 \pm 0.007$ & 11.32 \\
\hline TIP5P-Ew & $5.95 \times 10^{-5}$ & 3.34 & $0.620 \pm 0.007$ & 1.87 \\
\hline $\operatorname{Exp}^{[37]}$ & & & 0.610 & \\
\hline \multicolumn{5}{|c|}{ 318K } \\
\hline SPC & $6.91 \times 10^{-5}$ & 2.75 & $0.870 \pm 0.019$ & 36.96 \\
\hline SPC/E & $6.92 \times 10^{-5}$ & 2.56 & $0.940 \pm 0.016$ & 47.53 \\
\hline TIP3P & $7.02 \times 10^{-5}$ & 2.57 & $0.950 \pm 0.023$ & 49.08 \\
\hline TIP4P & $6.83 \times 10^{-5}$ & 2.76 & $0.860 \pm 0.015$ & 34.87 \\
\hline $\begin{array}{c}\text { TIP4P- } \\
\text { Ew }\end{array}$ & $6.79 \times 10^{-5}$ & 2.50 & $0.940 \pm 0.017$ & 48.42 \\
\hline $\begin{array}{c}\text { TIP4P- } \\
2005\end{array}$ & $6.79 \times 10^{-5}$ & 2.73 & $0.860 \pm 0.013$ & 35.81 \\
\hline TIP5P & $6.54 \times 10^{-5}$ & 3.43 & $0.660 \pm 0.006$ & 4.08 \\
\hline $\begin{array}{c}\text { TIP5P- } \\
\text { Ew }\end{array}$ & $6.44 \times 10^{-5}$ & 3.26 & $0.680 \pm 0.004$ & 7.59 \\
\hline $\operatorname{Exp}^{[37]}$ & & & 0.630 & \\
\hline
\end{tabular}

The velocity gradient of TIP4P/2005 at $318 \mathrm{~K}$ is shown in Figure 2-2, where the linear relation between $v_{x}$ and $z$ can be observed. Table 2-4 summarizes the shear viscosity of each model at $298 \mathrm{~K}$ and $318 \mathrm{~K}$, respectively. It can be seen that TIP5P and TIP5P-Ew still showed the best performances, with relative error ranging from $8.61 \%$ to $12.03 \%$ (relatively larger than thermal conductivity results), in comparison with other models, at $298 \mathrm{~K}$. For the cases at $318 \mathrm{~K}$, the performances are even better with small and stable relative error, $10.91 \%$ and $12.81 \%$, respectively. The results indicate that all of eight rigid 
models can predict decreasing trends of shear viscosity with increasing temperature. It is worthwhile to mention that shear viscosity obtained from RNEMD is close to that from Green-Kubo method for some models, compare to the value in reference[24].The values from reference 10 is also listed in Table 2-4 (column 4, in the parenthesis). For examples, the value of TIP3P from RNEMD is $0.318 \pm 0.0041 \mathrm{~m} \mathrm{~Pa} \cdot \mathrm{s}$, while the result from GreenKubo method is $0.321 \mathrm{~m} \mathrm{~Pa} \cdot \mathrm{s}$. Meanwhile, the viscosity obtained using TIP4P is $0.417 \pm 0.0072 \mathrm{~m} \mathrm{~Pa} \cdot \mathrm{s}$, while the value obtained from Green-Kubo method is $0.494 \mathrm{~m} \mathrm{~Pa} \cdot$ $\mathrm{s}$. When TIP5P model is used in the RNEMD simulation, the viscosity is $0.783 \pm 0.0504 \mathrm{~m}$ $\mathrm{Pa} \cdot \mathrm{s}$ whereas Green-Kubo method yields $0.699 \mathrm{~m} \mathrm{~Pa} \cdot \mathrm{s}$. For SPC model and TIP4P-2005 model, the differences, which are relatively larger, are $-54.2 \%$ and $-32.7 \%$, respectively. Moreover the result based on Green-Kubo method shows that TIP4P/2005 is the best model for shear viscosity prediction at $298 \mathrm{~K}$, which is different from the conclusion obtained from RNEMD. Since RNEMD is an approach close to the real experiment technique; the obtained results are still meaningful that can be a guide for other nonequilibrium molecular dynamics simulations treating with those systems composed with rigid-water molecules. 


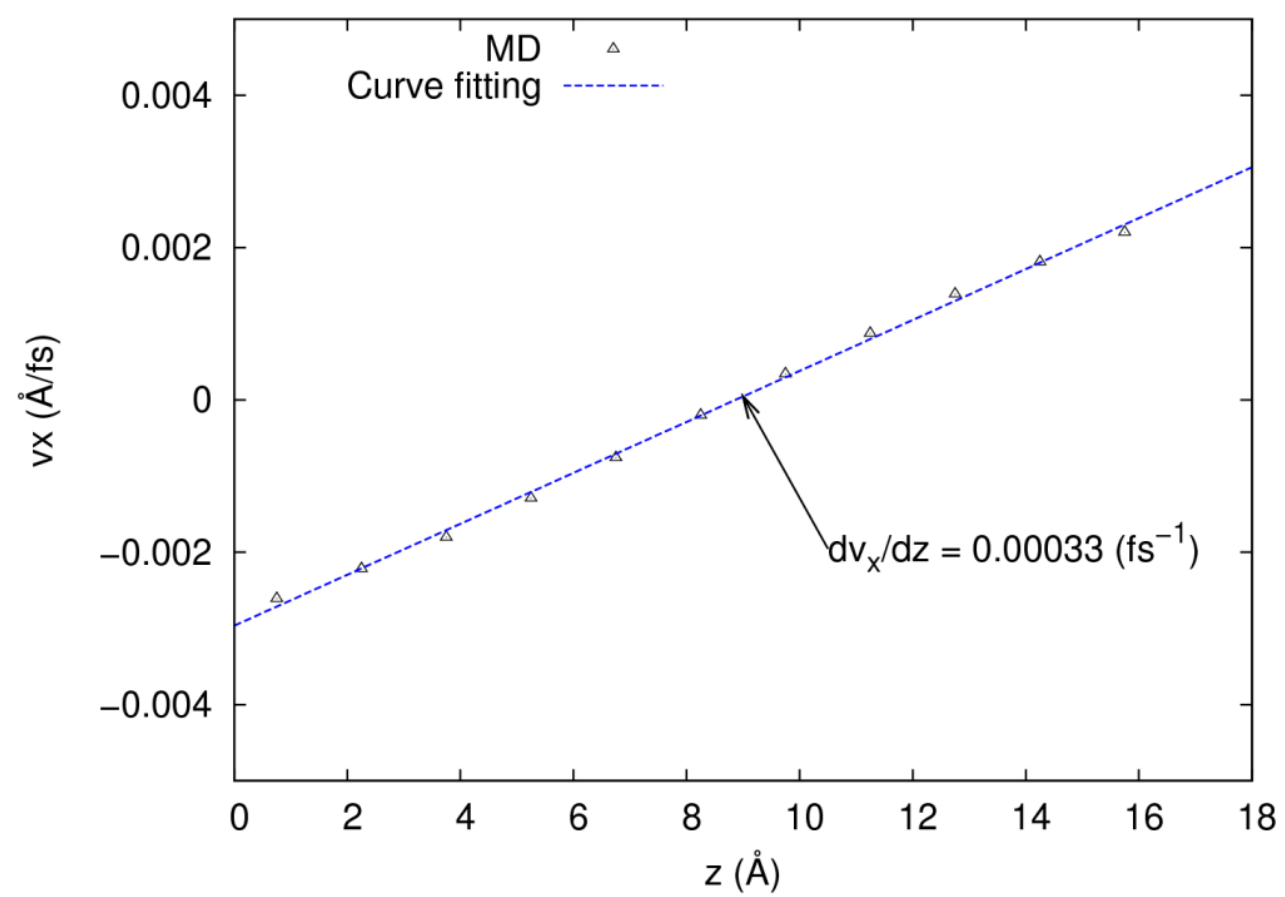

Figure 2-2 Established velocity gradient for TIP4P/2005 water system at $318 \mathrm{~K}$

Specific heat simulations are also performed in NVT and NPT ensemble within the same simulation boxes. The internal energy and enthalpy are obtained during the gradual cooling process in the simulation boxes from $343 \mathrm{~K}$ to $283 \mathrm{~K}$. The relationship between enthalpy (internal energy) and temperature for SPC/E (TIP4P-Ew) model is shown in Figure 2-3(Figure 2-4). 


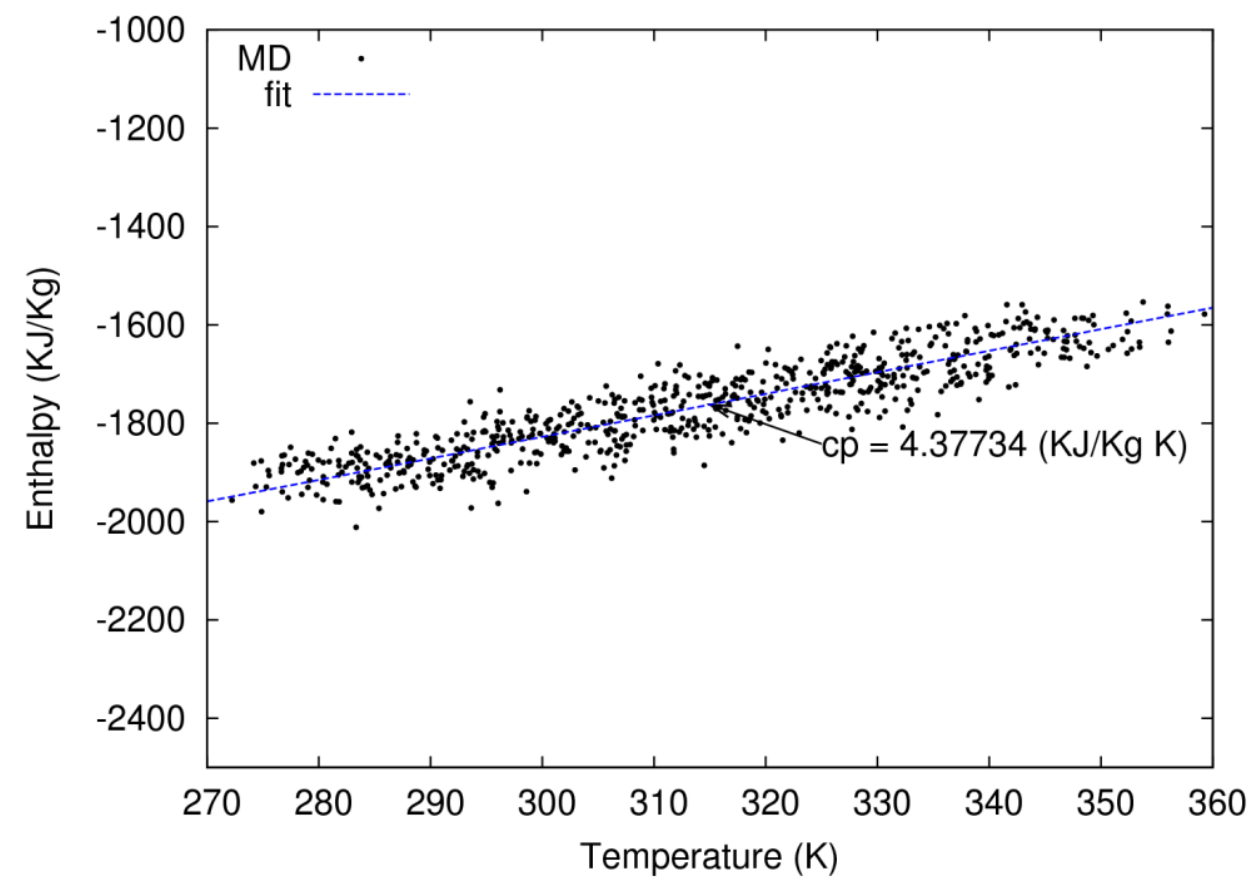

Figure 2-3 Relation between enthalpy and temperature for SPC/E water system

Figure 2-3 indicates the linear relationship between enthalpy and temperature, while Figure 2-4 shows the similar relation between internal energy and temperature.

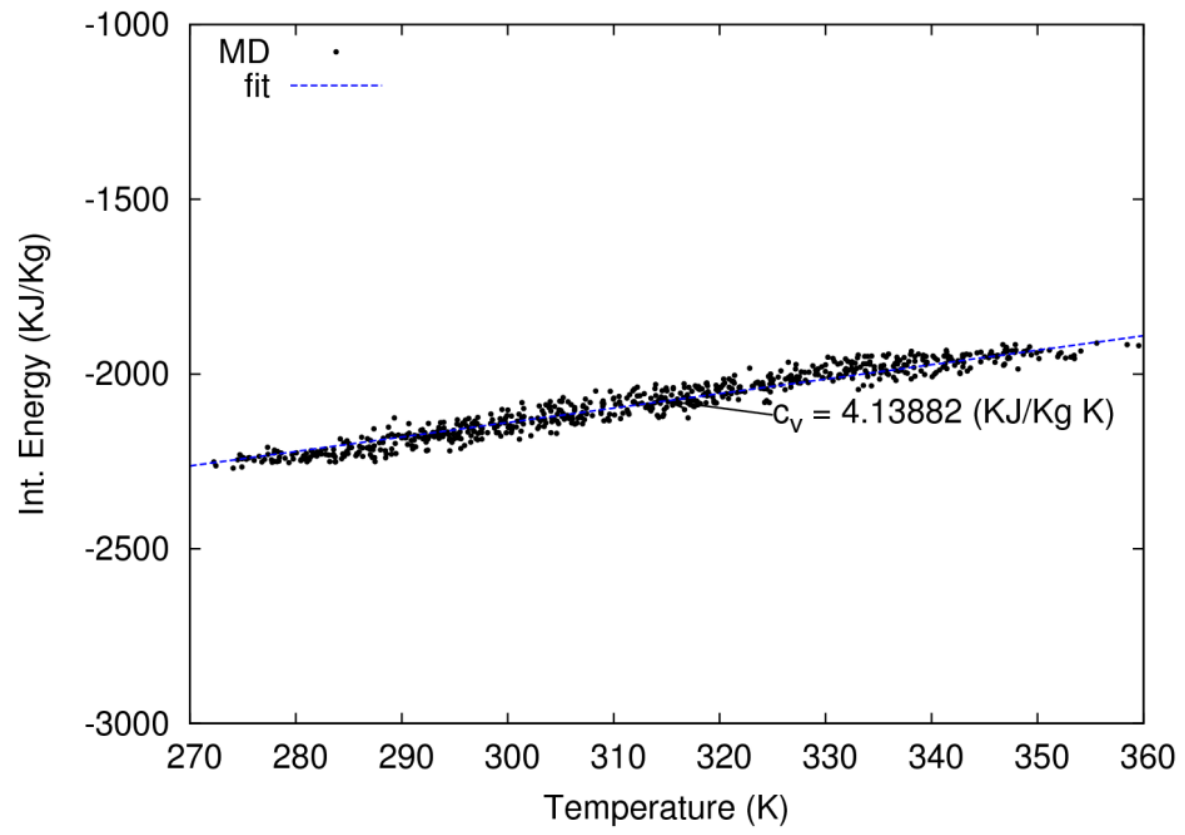

Figure 2-4 Relation between internal energy and temperature for TIP4P-Ew water system 
The values of specific heats are corresponding to the slopes of Figure 2-3 and Figure $2-4$, since the assumed linear relationship is applied. The obtained specific heats are given in Table 2-4.

Table 2-4 Shear viscosities at $298 \mathrm{~K}$ and $318 \mathrm{~K}$

\begin{tabular}{|c|c|c|c|c|}
\hline \multicolumn{5}{|c|}{ 298K } \\
\hline & $\begin{array}{c}\text { momentum } \\
\text { flux } \\
\left(\mathrm{g} \mathrm{mole}^{-1} \AA^{-1}\right. \\
\left.\text { fs }^{-2}\right)\end{array}$ & $\begin{array}{c}\text { velocity } \\
\text { gradient } \\
\left(\AA \mathbf{f s}^{-1}\right)\end{array}$ & $\begin{array}{l}\text { shear viscosity } \\
(\mathbf{P a} \cdot \mathbf{s})\end{array}$ & $\begin{array}{c}\text { relative } \\
\text { error } \\
\%\end{array}$ \\
\hline SPC & $1.25 \times 10^{-5}$ & $3.81 \times 10^{-4}$ & $(2.720 \pm 0.026) \times 10^{-4}$ & 69.49 \\
\hline $\mathrm{SPC} / \mathrm{E}$ & $1.45 \times 10^{-5}$ & $3.61 \times 10^{-4}$ & $\begin{array}{c}(3.340 \pm 0.038) \times 10^{-} \\
4\left(7.290 \times 10^{-4}\right)\end{array}$ & 62.53 \\
\hline TIP3P & $1.41 \times 10^{-5}$ & $3.67 \times 10^{-4}$ & $\begin{array}{c}(3.180 \pm 0.041) \times 10^{-} \\
4\left(3.21 \times 10^{-4}\right)\end{array}$ & 64.23 \\
\hline TIP4P & $1.72 \times 10^{-5}$ & $3.42 \times 10^{-4}$ & $\begin{array}{c}(4.17 \pm 0.072) \times 10^{-} \\
4\left(4.940 \times 10^{-4}\right)\end{array}$ & 53.15 \\
\hline $\begin{array}{l}\text { TIP4P- } \\
\text { Ew }\end{array}$ & $1.99 \times 10^{-5}$ & $2.89 \times 10^{-4}$ & $\begin{array}{c}(5.730 \pm 0.096) \times 10^{-} \\
4\left(8.550 \times 10^{-4}\right)\end{array}$ & 35.61 \\
\hline $\begin{array}{l}\text { TIP4P- } \\
2005\end{array}$ & $2.09 \times 10^{-5}$ & $3.01 \times 10^{-4}$ & $(5.750 \pm 0.085) \times 10^{-4}$ & 35.37 \\
\hline TIP5P & $2.68 \times 10^{-5}$ & $2.84 \times 10^{-4}$ & $\begin{array}{c}(7.830 \pm 0.504) \times 10^{-} \\
4\left(6.99 \times 10^{-4}\right)\end{array}$ & 12.03 \\
\hline $\begin{array}{l}\text { TIP5P- } \\
\text { Ew }\end{array}$ & $2.66 \times 10^{-5}$ & $2.71 \times 10^{-4}$ & $(8.130 \pm 0.512) \times 10^{-4}$ & 8.61 \\
\hline $\operatorname{Exp}^{[25]}$ & & & $8.900 \times 10^{-4}$ & \\
\hline \multicolumn{5}{|c|}{ 318K } \\
\hline SPC & $1.18 \times 10^{-5}$ & $4.04 \times 10^{-4}$ & $(2.420 \pm 0.022) \times 10^{-4}$ & 60.04 \\
\hline SPC/E & $1.36 \times 10^{-5}$ & $3.86 \times 10^{-4}$ & $(2.910 \pm 0.030) \times 10^{-4}$ & 51.93 \\
\hline TIP3P & $1.31 \times 10^{-5}$ & $3.93 \times 10^{-4}$ & $(2.780 \pm 0.030) \times 10^{-4}$ & 54.22 \\
\hline TIP4P & $1.57 \times 10^{-5}$ & $3.74 \times 10^{-4}$ & $(3.480 \pm 0.052) \times 10^{-4}$ & 42.59 \\
\hline $\begin{array}{l}\text { TIP4P- } \\
\text { Ew }\end{array}$ & $1.84 \times 10^{-5}$ & $3.34 \times 10^{-4}$ & $(4.580 \pm 0.073) \times 10^{-4}$ & 24.51 \\
\hline $\begin{array}{l}\text { TIP4P- } \\
2005\end{array}$ & $1.93 \times 10^{-5}$ & $3.37 \times 10^{-4}$ & $(4.760 \pm 0.071) \times 10^{-4}$ & 21.56 \\
\hline TIP5P & $2.60 \times 10^{-5}$ & $3.15 \times 10^{-4}$ & $(6.840 \pm 0.461) \times 10^{-4}$ & 12.81 \\
\hline $\begin{array}{l}\text { TIP5P- } \\
\text { Ew }\end{array}$ & $2.59 \times 10^{-5}$ & $3.20 \times 10^{-4}$ & $(6.720 \pm 0.486) \times 10^{-4}$ & 10.91 \\
\hline $\operatorname{Exp}{ }^{[25]}$ & & & $6.060 \times 10^{-4}$ & \\
\hline
\end{tabular}

It is interesting to note that three-site models (SPC, SPC/E, TIP3P) come out to be 
the best models to predict the specific heat at constant volume, while TIP5P and TIP5PEw overestimated the values much more than others. The value of specific heat at constant pressure predicted by SPC/E, TIP3P and TIP4P agree well with experimental results.

Table 2-5 specific heats

\begin{tabular}{|c|c|c|c|c|}
\hline & $\begin{array}{c}\mathrm{c}_{\mathbf{p}} \\
\left(\mathrm{KJ} \mathrm{Kg}^{-1} \mathbf{K}^{-1}\right)\end{array}$ & $\begin{array}{c}\text { relative error } \\
\%\end{array}$ & $\begin{array}{c}\mathrm{c}_{\mathrm{v}} \\
\left(\mathrm{KJ} \mathrm{Kg}^{-1} \mathrm{~K}^{-1}\right)\end{array}$ & $\begin{array}{c}\text { relative error } \\
\% \\
\end{array}$ \\
\hline SPC & 4.25591 & 1.82 & 3.46096 & 17.20 \\
\hline $\mathrm{SPC} / \mathrm{E}$ & 4.51339 & 7.98 & 4.12349 & 1.35 \\
\hline TIP3P & 4.48039 & 7.19 & 4.14095 & 0.93 \\
\hline TIP4P & 4.93893 & 18.16 & 4.55668 & 9.01 \\
\hline TIP4P-Ew & 4.79089 & 14.61 & 4.91585 & 17.60 \\
\hline TIP4P-2005 & 4.99979 & 19.61 & 5.14207 & 23.02 \\
\hline TIP5P & 5.02839 & 20.30 & 6.56332 & 57.02 \\
\hline TIP5P-Ew & 5.90856 & 41.35 & 5.65392 & 35.26 \\
\hline $\operatorname{Exp}^{[25]}$ & 4.18 & & 4.18 & \\
\hline
\end{tabular}

However, the consistency between two specific heats is not well. If taking all these results together, it can be found that three-site model, TIP3P, turns to be the best in prediction both specific heats.

\subsection{Conclusion}

In conclusion, if molecular dynamics method is used to simulate heat transfer for a rigid water molecular system, the five-site models (TIP5P-Ew) are better choices. For dynamic problem that involves water flow in micro and nano-systems, the five-site models (TIP5P, TIP5P-Ew) are also appropriate choices. If a problem emphasizing dynamic response of the temperature to a heatwelling or cooling process, the three-site models are good candidates, especially TIP3P model.

\section{Acknowledgement}


Support for this work by the U.S. National Science Foundation under grant number CBET- 1066917 is gratefully acknowledged. 


\section{Chapter 3 Prediction of the Temperature-Dependent Thermal Conductivity and Shear Viscosity for Rigid Water Models}

\subsection{Introduction}

Water properties have been intensively investigated in the past decades, at the molecular simulation level - both from quantum (AIMD)[38] and classical molecular dynamics. Many types of water models are proposed with the purpose of reproducing the properties as much as possible. A 2002 literature review indicates that there are 46 water models[15], which are categorized into rigid, flexible and polarizable models[16]. Recently, new well-performance models which are reparameterized based on the existing ones are proposed through further studies. For instance, TIP4P/2005[17] that is designed to be a general purpose model for condensed phase of water has an impressive performance in predicting a variety of thermal properties. Another extended version of four-site rigid water model, TIP4P-Ew[18], has a global improvement in predicting water properties. TIP5P-Ew[19] that was reparameterized based on TIP5P model has been reported that it has great performance in reproducing experiment data for liquid water.

However, none of the above models has the capability to perfectly reproduce all properties of water, which leads to confusion when researchers need to choose an appropriate model before conducting their molecular dynamics simulations in terms of different problems. For example, the thermal properties and structure of clathrate hydrates highly rely on what water model is used[39]. Moreover, although various properties are investigated, and advantages and disadvantages are also reported in the literature, independent papers that report the performances among these widely used 
models on reproducing thermophysical and dynamic properties are seldom. Equilibrium and dynamic properties, including specific heat and thermal conductivity, are reported by Bertolini[40] for SPC/E and TIP4P. Mark and Nilsson[41] investigated both structure and dynamics of three-site models, TIP3P, SPC and SPC/E at 298K. González and Abascal [24]calculated the shear viscosity with Green-Kubo model and compare the results obtained from five rigid water models that include TIP3P[21], TIP4P[21], TIP5P[22], SPC/E[23] and TIP4P/2005with the experimental data. However, evaluations of different models on predicting thermal conductivity have not been done, although it is a very important property in thermal and hydrodynamic problems.

There exist two main methods, equilibrium molecular dynamics (EMD) and nonequilibrium molecular dynamics (NEMD), to determine shear viscosity and thermal conductivity. Green-Kubo[25] based method using EMD in which transport properties are related to the time integral of a correlation function. It usually can predict the result with a reasonable accuracy and precision. However, it suffers from the difficulties on complications of determining the microscopic heat flux (momentum flux in case of shear viscosity) and slow convergence of the time integral of the heat flux (or momentum flux) autocorrelation function (1000-10000 times the decay time). For the NEMD, imposing gradient, either temperature or velocity gradient, is one choice, but still have same difficulties in calculating heat or momentum flux, which are especially difficult to determine in the charged systems. To overcome these limits of Green-Kubo method and NEMD, another non-equilibrium molecular dynamics approach - Reverse NonEquilibrium Molecular Dynamics (RNEMD) - has been developed by Müller-Plathe [26], which is also an accurate and efficient method for monoatomic molecular fluids 
[27]. Later, Bedrov and Smith[28] extended the RNEMD to be suitable for rigid polyatomic molecular fluids without violating conservation of energy and linear momentum of the system. In this method, flux, which is accumulated during simulation, is imposed to the system; gradient is just a response of corresponding flux. However, it should be pointed out that results obtained from RNEMD[42] somehow depend on swap frequency, and carefully choosing the value is required before running MD simulations. The authors' study has showed that the frequencies of 200 and 50 yielded fairly well predicted thermal conductivity and shear viscosity, respectively[43].

In this work, both thermal conductivity and shear viscosity will be calculated with RNEMD method for liquid water at temperatures ranging from $283 \mathrm{~K}$ to $363 \mathrm{~K}$. One reason of choosing RNEMD is that the operations of this approach is similar to real technique when measuring thermal or dynamic properties of fluids in the laboratories; thus it can physically reflect the properties of fluids in a "real" situation. Moreover, RNEMD is particularly attractive for systems involving long-range interaction where calculation of the microscopic heat flux (or momentum flux) is difficult. The following eight rigid models, SPC[30, 31], SPC/E, TIP3P, TIP4P, TIP4P/2005, TIP4P-Ew, TIP5P and TIP5-Ew, are selected to carry out these simulations in this work based on their relatively successful performance as reported in the literatures.

\subsection{Methods}

The RNEMD comprise of two main steps, which including generating flux in an unphysical way[34] and measuring corresponding gradient when steady-state is reached. 


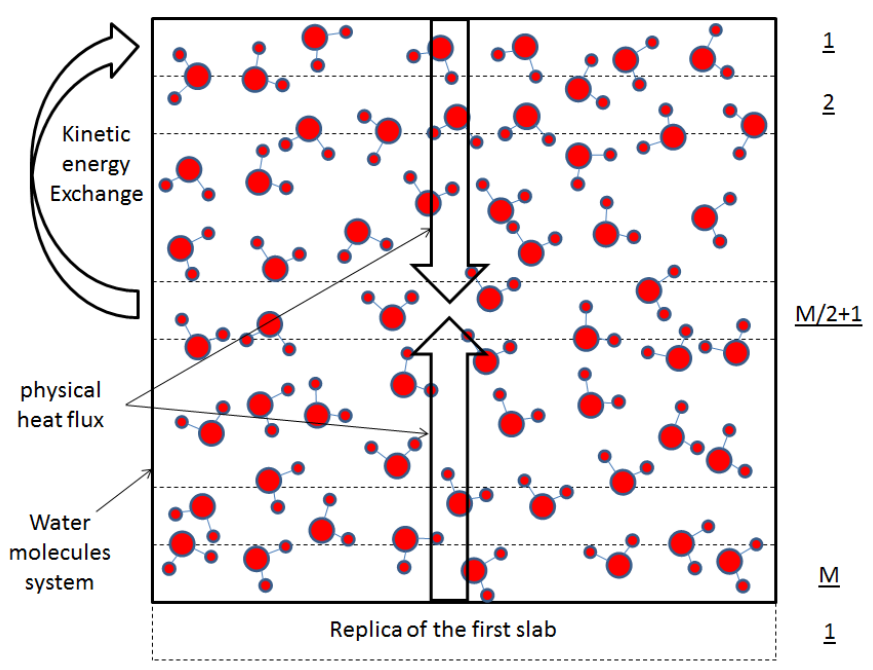

Figure 3-1 Schematic of reverse non-equilibrium molecular dynamics (RNEMD)

Figure 3-1 gives a 2D schematic of the RNEMD in calculating thermal conductivity. First, the box is uniformly divided into $\mathrm{M}$ (even number) slabs, as framed by the dashed lines. Then $n$ hottest molecules in $\mathrm{M} / 2+1$ slab and coldest ones in $1^{\text {st }}$ slab will be selected and paired; then the kinetic energy in each pair will be swapped. Thus, an unphysical heat flux flowing from $\mathrm{M} / 2+1$ slab to $1^{\text {st }}$ slab is generated. As a result, two physical heat fluxes, flowing from $1^{\text {st }}$ to $\mathrm{M} / 2+1$ and from $\mathrm{M}+1$ to $\mathrm{M} / 2+1$, are generated in response. If the kinetic energy is swapped periodically, a stable heat flux and a corresponding temperature gradient will be established. The final heat flux can be calculated as,

$$
q^{\prime \prime}=\frac{E_{k e, e x}}{2 t A}
$$

where $E_{k e, e x}$ denotes the exchanged kinetic energy between each pair, $A$ is area that heat flux flow across, and $t$ is the total simulation time. The exchanged kinetic energy is calculated by

$$
E_{k e, e x}=\sum_{i=1}^{n} \frac{1}{2} M_{i}\left(v_{\text {hot }, i}^{2}-v_{\text {cold }, i}^{2}\right)
$$


where $v_{h o t, i}$ and $v_{c o l d, i}$ are velocity at COM (center of mass) of hottest molecules and coldest ones, respectively; $M_{\mathrm{i}}$ is the total mass of single molecule; $n$ is pairs number of selected molecules. As reference[28] recommended, velocity at COM of each rigid molecule is exchanged while the relatively velocity to its COM of each atom keep unchanged in order to avoid additional perturbation in the system[28].

To measure temperature in each slab, degree of each rigid molecule should be carefully treated in order to avoid overestimation. Since it is a little difficult to estimate single rigid molecule-based temperature due to the possible situations that a single molecule spanning across two neighbor slabs, the temperature of each atom are calculated independently while an algorithm of excluding extra constrains in each atom are adopted[27]. Then, the slab temperatures are easily calculated by averaging all atoms within each slab.

After the system reaches to a steady-state, a symmetric temperature profile will be established, and it will be recorded during the sampling period. The thermal conductivity can then be obtained through Fourier's Law,

$$
k=-\frac{q^{\prime \prime}}{d T / d x_{i}}
$$

where $x_{i}$ could be $x, y$, and $z$. Temperature gradient and accumulated exchanged energy will be calculated with least-square fitting. The error bar will be calculated through,

$$
\Delta k=k\left(\frac{\Delta E_{k e, e x}}{E_{k e, e x}}+\frac{\Delta G_{T}}{G_{T}}\right)
$$

where, $\Delta E_{\mathrm{ke}, \mathrm{ex}}$ and $\Delta G_{T}$ are error bar from each linear fit, and $G_{T}$ represents temperature gradient in this fitting.

For shear viscosity calculation, similar approach is applied to the same system, except 
exchanging momentum instead of kinetic energy for pairs of molecules. The corresponding velocity gradient will be calculated through,

$$
j_{i}=\frac{P_{m o, e x, i}}{2 t A}
$$

where $P_{\mathrm{mo}, \mathrm{ex}, \mathrm{i}}$ represents the amount of exchanged momentum in $i$ component ( $i$ could be $x, y, z)$, which can be calculated as,

$$
P_{m o, e x, i}=\sum_{j=1}^{n} \frac{1}{2} M_{j}\left(v_{\text {pos } j, i}-v_{n e g j, i}\right)
$$

where $v_{\text {posj,i }}$ and $v_{\text {negj,i }}$ represent positive and negative velocity component in the $i$ direction (bulk velocity of the box is excluded) of $j^{\text {th }}$ molecule pair. Similarly, shear viscosity can be calculated through newton's law for fluid,

$$
\mu=-\frac{j_{i}}{d v_{i} / d x_{i}}
$$

where $i$ could be $x, y, z$. Error bar will estimated as,

$$
\Delta \mu=\mu\left(\frac{\Delta P_{m o, e x, i}}{P_{m o, e x, i}}+\frac{\Delta G_{v}}{G_{v}}\right)
$$

where $\Delta P_{i, e x}$ and $\Delta G_{v}$ are error bar from each linear fit, $G_{v}$ represents velocity gradient in its least-square fit.

In this work, eight different rigid water models will be studied; all potential functions of these rigid water molecules share the same form, which consist of the contributions from electrostatic, dispersion and repulsive forces:

$$
E_{a b}=\sum_{i}^{\text {on } a \text { on } b} \sum_{j}^{k_{C} q_{a_{i}} q_{b_{j}}}+\sum_{a_{i} b_{j}}^{\text {on a on } b} \sum_{j} 4 \varepsilon_{a_{i} b_{j}}\left[\left(\frac{\sigma_{a_{i} b_{j}}}{r_{a_{i} b_{j}}}\right)^{12}-\left(\frac{\sigma_{a_{i} b_{j}}}{r_{a_{i} b_{j}}}\right)^{6}\right]
$$

where, $a$ and $b$ denotes two different molecules, subscript $i$ and $j$ represent atom $i$ in one individual molecule for all three-site rigid water molecule, $k_{\mathrm{c}}$ is electrostatic constant. For 
four- and five-site rigid water models, $i$ and $j$ represent massless but charged site in the first summation, still denotes atom in the second summation. Short range force is neglected when the distance of two atoms exceeds the cutoff distance, but the electrostatic force is evaluated with PPPM method[32] that splits this effect into short range and long range parts. For four- and five-site models, Coulomb forces on virtual sites are redistributed to real atoms by adopting the algorithm in reference[44]. All parameters for different potentials are summarized in Table 3-1. 
Table 3-1 Parameters of 8 rigid water models

\begin{tabular}{|c|c|c|c|c|c|c|c|c|}
\hline Model & $\sigma(\AA)$ & $\varepsilon\left(\right.$ Kcal mole $\left.^{-1}\right)$ & $q H(e)$ & qo(e) & $\theta\left({ }^{\circ}\right)$ & $\varphi\left(^{\circ}\right)$ & $\mathbf{L}_{\mathbf{M}} / \mathbf{L L}_{L}(\AA)$ & $\mathbf{L}(\AA)$ \\
\hline SPC & 3.16600 & 0.15535 & 0.41000 & -0.82000 & 109.47 & $\mathrm{~N} / \mathrm{A}$ & $\mathrm{N} / \mathrm{A}$ & 1.00000 \\
\hline SPC/E & 3.16600 & 0.15535 & 0.42380 & -0.84760 & 109.47 & $\mathrm{~N} / \mathrm{A}$ & N/A & 1.00000 \\
\hline TIP3P & 3.15061 & 0.15210 & 0.41700 & -0.83400 & 104.52 & $\mathrm{~N} / \mathrm{A}$ & $\mathrm{N} / \mathrm{A}$ & 0.97520 \\
\hline TIP4P & 3.15365 & 0.16348 & 0.52000 & -1.04000 & 104.52 & $\mathrm{~N} / \mathrm{A}$ & 0.15000 & 0.97520 \\
\hline TIP4P-Ew & 3.16435 & 0.16275 & 0.52422 & -1.04844 & 104.52 & $\mathrm{~N} / \mathrm{A}$ & 0.12500 & 0.97520 \\
\hline TIP4P/2005 & 3.15890 & 0.18521 & 0.55640 & -1.11280 & 104.52 & N/A & 0.15460 & 0.97520 \\
\hline TIP5P & 3.12000 & 0.15999 & 0.24100 & -0.24100 & 104.52 & 109.47 & 0.70000 & 0.97520 \\
\hline TIP5P-Ew & 3.09700 & 0.17801 & 0.24100 & -0.24100 & 104.52 & 109.47 & 0.70000 & 0.97520 \\
\hline
\end{tabular}


All water molecular systems are treated in the same manner, except applying different potential, charge value, and geometrical parameters (see Table 3-1). A shake algorithm[33] is applied to each water molecule to make it as rigid. For transport properties evaluation, all the simulations have been performed in canonical ensemble (NVT) using the artificial mass based Nośe-Hoover thermostat[35] for a sample size of 900 water molecules cubic system, whose boundaries is treated as periodic with dimension of $30 \AA \times 30 \AA \times 30 \AA$. This box size is sufficient, since the size effect has been used and tested in reference[27]. Thus the density of liquid water is fixed at 996.67 $\mathrm{kg} / \mathrm{m}^{3}$. The simulation box is divided into $M=20$ slabs in the z-direction and 10 hottest molecules at bottom slab and coldest in middle slab will be selected to exchange kinetic energy (or momentum in the $x$-direction for shear viscosity calculation). The simulation time step is $1 \mathrm{fs}$ and the total simulation time is $2 \mathrm{~ns}$, excluding the equilibration period of $0.2 \mathrm{~ns}$. The cut-off distance for short range force is $1.04 \mathrm{~nm}$. In order to achieve a reasonable linear response, swap frequencies of 200 and 50 are carefully chosen for thermal conductivity and shear viscosity calculations, respectively. The sample times of calculating gradients are $200 \mathrm{ps.}$

In order to carry out simulation within the framework of the open-source molecular dynamics software LAMMPS[36], RNEMD for thermal conductivity and shear viscosity for polyatomic molecule fluid are implemented. Meanwhile, five-site rigid water models are also available with PPPM/TIP5P solver to obtain long range coulomb force, some additional function including counting degrees of freedom for single atom are also added into the original codes.

In addition, it is worth to notice that though TIP4P-Ew and TIP5P-Ew are designed to 
be modeled with Ewald summation technique, all these long range coulomb interactions are solved with PPPM method, since it can well approach the same precision of Ewald summation when mesh grids increased to reasonable number. In order to obtain the mesh size for PPPM solver, shear viscosity and thermal conductivity are calculated with both standard Ewald summation and PPPM solver for a test case. The relative errors for each value are listed in Table 3-2, with mesh size equals to 30 in the $x$-, $y$ - and $z$-directions. The results indicate that $30 \times 30 \times 30$ is sufficient to achieve the same precision as the standard Ewald summation.

Table 3-2 Comparison of results from different Kspace Solver

\begin{tabular}{|c|c|c|c|}
\hline $298 \mathrm{~K} / \mathrm{SPC}$ & Ewald & PPPM & $\mathbf{R E}$ \\
\hline $\mathbf{k}\left(\mathbf{W} \mathbf{m}^{-1} \mathbf{K}^{-1}\right)$ & 0.89 & 0.88 & $2.01 \%$ \\
\hline$\mu(\mathrm{mPa} \cdot \mathbf{s})$ & 0.281 & $0.272^{1}$ & $3.32 \%$ \\
\hline
\end{tabular}

\subsection{Results and Discussions}

In this work, the linear response of temperature distribution as a result of imposed heat flux to the system is well established. Figure 3-2 shows the final temperature profiles for each model at different temperatures. Since the temperature profiles are symmetric in the simulation box, the points in Figure 3-2 are obtained by averaging over all pairs of temperature values in the corresponding two symmetric slabs. All of the data during the sampling period are also averaged in order to obtain a statistically reasonable result. It can be seen that the linear profiles have been well established and the middle point of each line locate at expected temperature of the system. For example, the middle points of all cases in Figure 3-2(a) are sitting at 283K. It could also be noticed that both five-site models' temperature gradients are larger than others, while that from four-site and threesite models are very close to each other. 


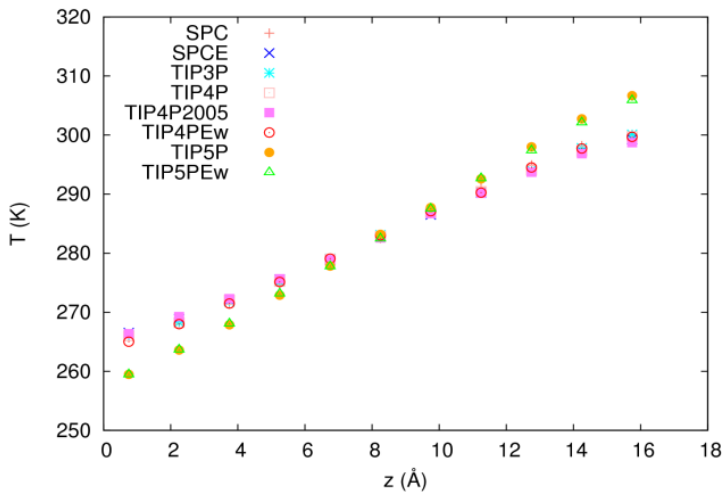

(a) $283 \mathrm{~K}$

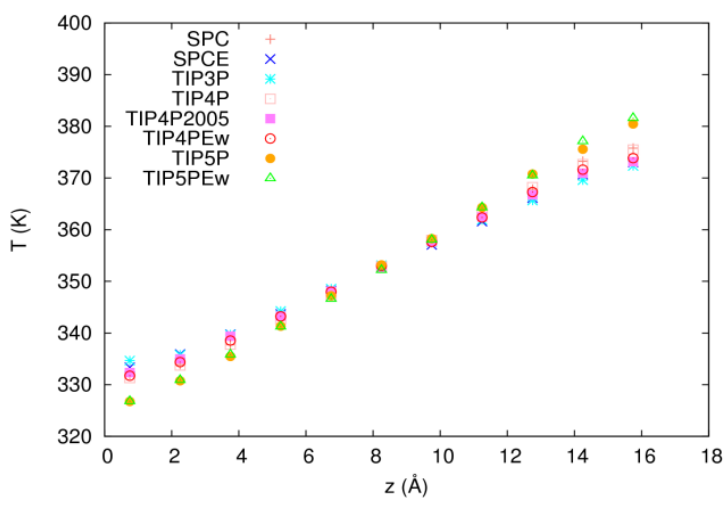

(c) $353 \mathrm{~K}$

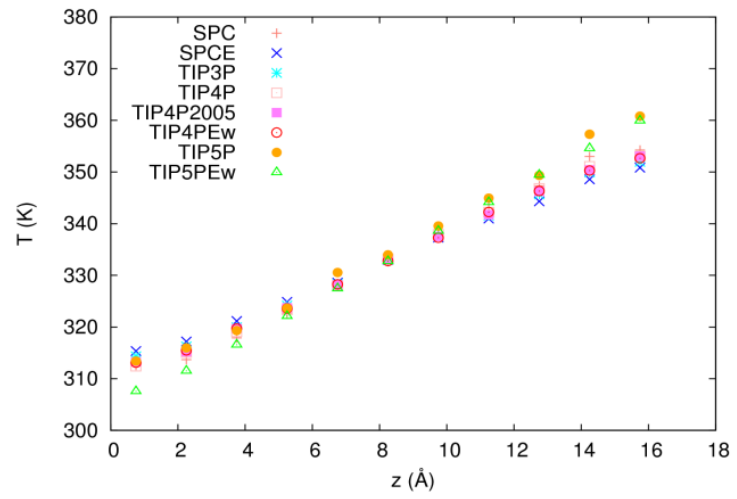

(b) $333 \mathrm{~K}$

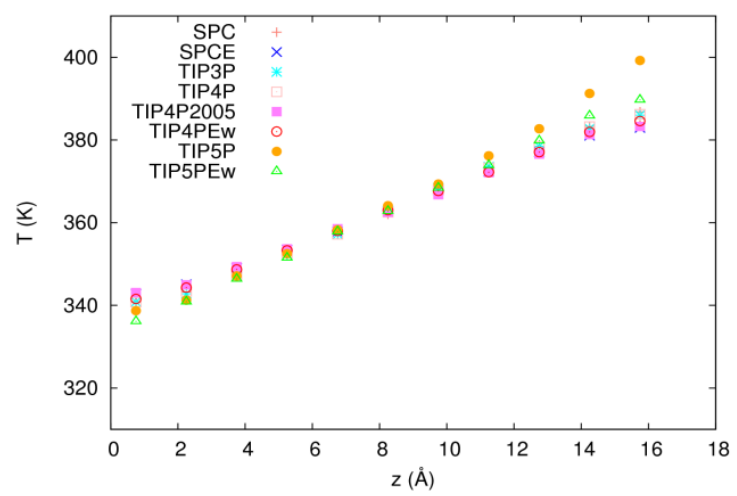

(d) $363 \mathrm{~K}$

Figure 3-2 Temperature profiles in simulation box for each rigid model at temperatures ranging from $283 \mathrm{~K}$ to $363 \mathrm{~K}$

Figure 3-3 gives the final results of thermal conductivities for each model at various temperatures. The solid-black triangle represents the data from the experiments [37]. It can be seen both five-site models have better performances than the rest of models at all temperatures. Minor discrepancies exist between TIP5P and TIP5P-Ew when temperature is $298 \mathrm{~K}, 313 \mathrm{~K}$, and $363 \mathrm{~K}$. It is interesting to notice that five-site models, either TIP5P or TIP5P-Ew, can always reproduce experimental value. Therefore, five-site models are the priority choices when thermal conductivity of the system is an essential factor determining the precision of the MD simulation. However, it should also been pointed out 
that the trends of thermal conductivity versus temperature from TIP5P and TIP5P-Ew are not perfectly ideal, comparing with the increasing trend that experimental values hold. Comparing with five-site, both four-site and three-site models over predict the value by tens of percent. Similar trends from results obtained from the five-site models can also be observed. For example, the thermal conductivity obtained from TIP4P model decreases when temperature increases to $298 \mathrm{~K}$ from $283 \mathrm{~K}$. Therefore, it can be concluded that none of a single rigid water model can reproduce the trends of experimental data of thermal conductivity.

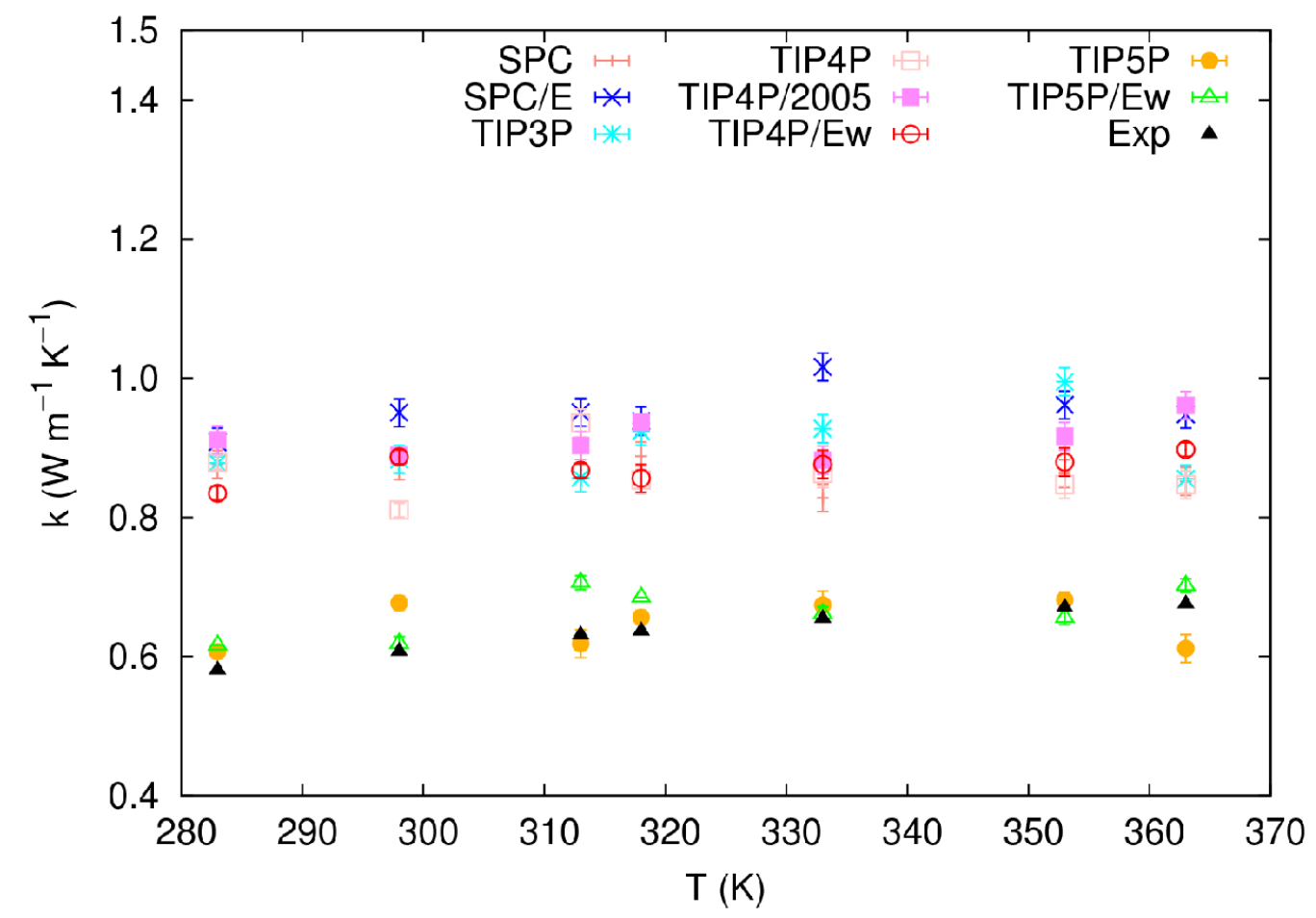

Figure 3-3 Thermal conductivities trends predicted by eight rigid models

Table 3-3 summarizes the thermal conductivities, its uncertainties and relative error to experimental value of all results showed in Figure 3-3. It can be seen that the uncertainties of all cases are within $3.35 \%$, which mean that the results in this work is very reliable. As discussed above, five-site models perform best with comparatively small 
relative errors. For TIP5P, it reaches to the maximum error of $11.50 \%$ (overestimate) at $298 \mathrm{~K}$, and minimum error of $1.97 \%$ (underestimate) at $313 \mathrm{~K}$; for TIP5P-Ew, the maximum error of $11.99 \%$ (overestimate) happen at $313 \mathrm{~K}$, and minimum of $1.06 \%$ (overestimate) at $333 \mathrm{~K}$. 
Table 3-3 Thermal conductivities of different model at various temperatures (W m-1 K-1)

\begin{tabular}{|c|c|c|c|c|c|c|c|c|c|}
\hline $\mathbf{k}$ & SPC & SPC/E & TIP3P & TIP4P & TIP4P-2005 & TIP4P-Ew & TIP5P & TIP5P-Ew & Exp. \\
\hline $283 K$ & $0.876 \pm 0.022$ & $0.908 \pm 0.016$ & $0.878 \pm 0.014$ & $0.879 \pm 0.013$ & $0.912 \pm 0.016$ & $0.835 \pm 0.014$ & $0.607 \pm 0.005$ & $0.616 \pm 0.005$ & 0.581 \\
\hline Err & $50.95 \%$ & $56.44 \%$ & $51.25 \%$ & $51.44 \%$ & $57.04 \%$ & $43.76 \%$ & $4.53 \%$ & $6.13 \%$ & \\
\hline $298 \mathrm{~K}$ & $0.874 \pm 0.020$ & $0.951 \pm 0.017$ & $0.883 \pm 0.019$ & $0.811 \pm 0.015$ & $0.889 \pm 0.014$ & $0.887 \pm 0.013$ & $0.677 \pm 0.007$ & $0.619 \pm 0.007$ & 0.607 \\
\hline Err & $44.01 \%$ & $56.55 \%$ & $45.48 \%$ & $33.49 \%$ & $46.44 \%$ & $46.06 \%$ & $11.50 \%$ & $1.87 \%$ & \\
\hline $313 \mathrm{~K}$ & $0.877 \pm 0.021$ & $0.951 \pm 0.019$ & $0.857 \pm 0.016$ & $0.935 \pm 0.016$ & $0.903 \pm 0.016$ & $0.868 \pm 0.010$ & $0.618 \pm 0.019$ & $0.706 \pm 0.007$ & 0.631 \\
\hline Err & $39.02 \%$ & $50.81 \%$ & $35.86 \%$ & $48.33 \%$ & $43.23 \%$ & $37.56 \%$ & $-1.97 \%$ & $11.99 \%$ & \\
\hline $318 \mathrm{~K}$ & $0.888 \pm 0.020$ & $0.938 \pm 0.016$ & $0.924 \pm 0.023$ & $0.854 \pm 0.015$ & $0.937 \pm 0.014$ & $0.856 \pm 0.015$ & $0.656 \pm 0.006$ & $0.685 \pm 0.004$ & 0.637 \\
\hline Err & $39.51 \%$ & $47.43 \%$ & $45.11 \%$ & & & & & & \\
\hline $333 \mathrm{~K}$ & $0.828 \pm 0.025$ & $1.016 \pm 0.019$ & $0.9 \eta \pm 0.020$ & $0.863 \pm 0.018$ & $0.882 \pm 0.017$ & $0.876 \pm 0.017$ & $0.674 \pm 0.021$ & $0.661 \pm 0.006$ & 0.654 \\
\hline Err & $26.53 \%$ & $55.29 \%$ & $41.72 \%$ & $31.84 \%$ & $34.85 \%$ & $33.92 \%$ & $3.02 \%$ & $1.06 \%$ & \\
\hline $353 \mathrm{~K}$ & $0.863 \pm 0.021$ & $0.962 \pm 0.017$ & $0.995 \pm 0.024$ & $0.847 \pm 0.019$ & $0.916 \pm 0.018$ & $0.880 \pm 0.017$ & $0.681 \pm 0.009$ & $0.656 \pm 0.011$ & 0.670 \\
\hline Err & $28.78 \%$ & $43.53 \%$ & $48.49 \%$ & $26.43 \%$ & $36.79 \%$ & $31.27 \%$ & $1.71 \%$ & $-2.14 \%$ & \\
\hline $363 \mathrm{~K}$ & $0.852 \pm 0.019$ & $0.948 \pm 0.023$ & $0.855 \pm 0.019$ & $0.847 \pm 0.018$ & $0.961 \pm 0.021$ & $0.897 \pm 0.014$ & $0.612 \pm 0.020$ & $0.702 \pm 0.006$ & 0.675 \\
\hline Err & $26.10 \%$ & $40.41 \%$ & $26.65 \%$ & $25.41 \%$ & $42.30 \%$ & $32.90 \%$ & $-9.44 \%$ & $3.94 \%$ & \\
\hline
\end{tabular}


For the shear viscosity, Figure 3-4 shows the linear response of formed profile of velocity in the $x$ - component that excludes the bulk velocity of the simulation box at steady-state. The maximum and minimum velocities respectively are $\sim 3 \AA /$ ps and $\sim-3$ $\AA / \mathrm{ps}$, and the middle points of the velocity distribution line locate at 0 due to exclusion of bulk velocity of the simulation box. Different from temperature profile in thermal conductivity calculation, the three-site models (TIP3P, SPC and SPC/E) have a larger gradient than others. Meanwhile, both four-site and three-site models have good linear responses; however, five-site models showed the worst linear responses. It has to be pointed out that swapping frequency in RNEMD can lead to such nonlinear profiles. The response can be improved by decreasing the frequency, but it was not changed because the value of 50 comes from a compromise of eight rigid models. 


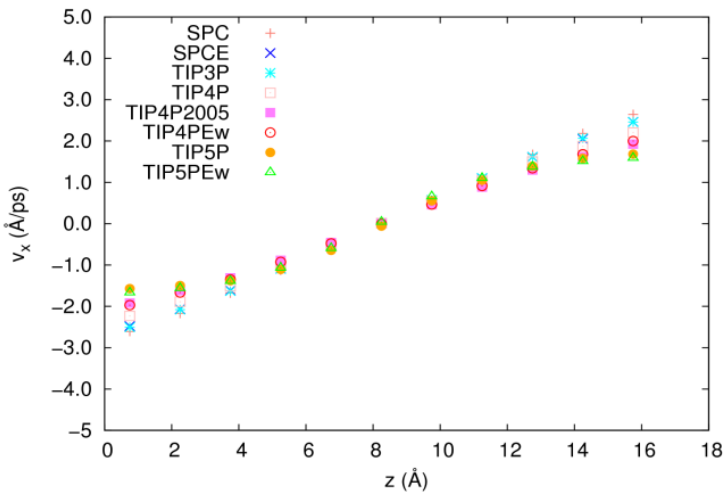

(a) $283 \mathrm{~K}$

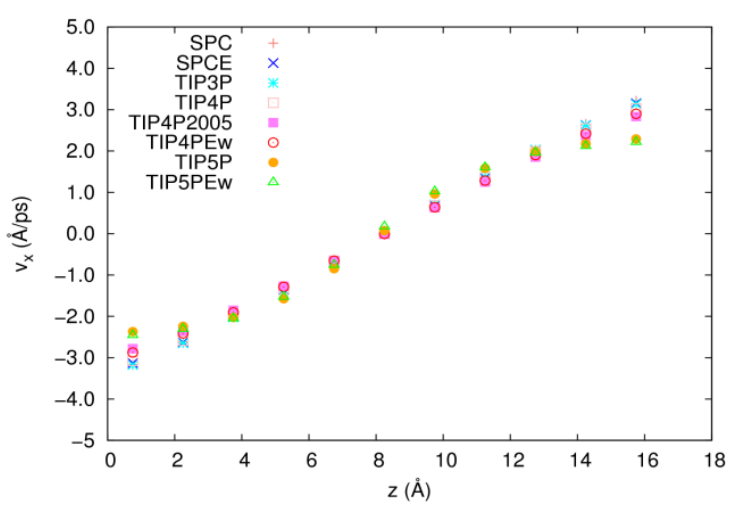

(c) $353 \mathrm{~K}$

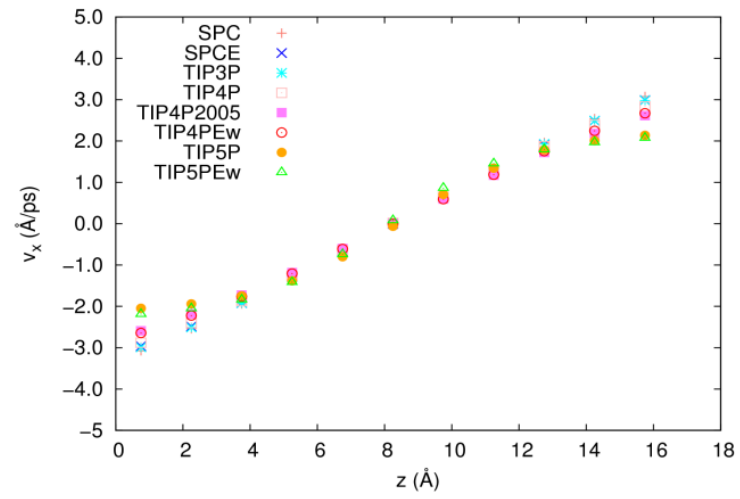

(b) $333 \mathrm{~K}$

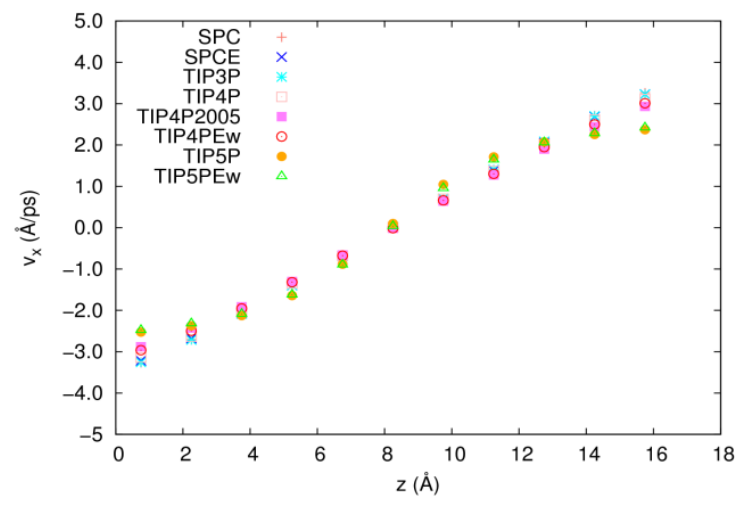

(d) $363 \mathrm{~K}$

Figure 3-4 velocity profiles (in $\mathrm{x}$ component) for each rigid model at temperatures ranging from $283 \mathrm{~K}$ to $363 \mathrm{~K}$ 
Table 3-4 Shear viscosities of different model at various temperatures ( $\mathrm{mPa} \cdot \mathrm{s})$

\begin{tabular}{|c|c|c|c|c|c|c|c|c|c|}
\hline$\mu$ & SPC & SPC/E & TIP3P & TIP4P & TIP4P-2005 & TIP4P-Ew & TIP5P & TIP5P-Ew & Exp. \\
\hline 283 & $0.300 \pm 0.003$ & $0.376 \pm 0.005$ & $0.362 \pm 0.005$ & $0.490 \pm 0.010$ & $0.681 \pm 0.013$ & $0.635 \pm 0.012$ & $0.882 \pm 0.059$ & $0.872 \pm 0.060$ & 1.306 \\
\hline Err & $-77.01 \%$ & $-71.21 \%$ & $-72.25 \%$ & $-62.50 \%$ & $-47.85 \%$ & $-51.37 \%$ & $-32.49 \%$ & $-33.24 \%$ & \\
\hline 298 & $0.272 \pm 0.005$ & $0.336 \pm 0.004$ & $0.318 \pm 0.004$ & $0.417 \pm 0.007$ & $0.599 \pm 0.009$ & $0.554 \pm 0.009$ & $0.767 \pm 0.058$ & $0.813 \pm 0.055$ & 0.890 \\
\hline Err & $-69.44 \%$ & $-62.27 \%$ & $-64.23 \%$ & $-53.15 \%$ & $-32.68 \%$ & $-37.74 \%$ & $-13.81 \%$ & $-8.61 \%$ & \\
\hline 313 & $0.249 \pm 0.002$ & $0.297 \pm 0.003$ & $0.285 \pm 0.003$ & $0.366 \pm 0.006$ & $0.503 \pm 0.008$ & $0.462 \pm 0.008$ & $0.749 \pm 0.047$ & $0.668 \pm 0.053$ & 0.653 \\
\hline Err & $-61.80 \%$ & $-54.52 \%$ & $-56.33 \%$ & $-43.94 \%$ & $-22.98 \%$ & $-29.16 \%$ & $14.81 \%$ & $2.41 \%$ & \\
\hline 318 & $0.253 \pm 0.005$ & $0.298 \pm 0.003$ & $0.278 \pm 0.003$ & $0.348 \pm 0.005$ & $0.480 \pm 0.007$ & $0.454 \pm 0.007$ & $0.724 \pm 0.045$ & $0.672 \pm 0.052$ & 0.606 \\
\hline Err & $-58.25 \%$ & $-50.91 \%$ & $-54.22 \%$ & $-42.59 \%$ & $-20.85 \%$ & $-25.18 \%$ & $19.46 \%$ & & \\
\hline 333 & $0.226 \pm 0.002$ & $0.264 \pm 0.003$ & $0.253 \pm 0.003$ & $0.308 \pm 0.004$ & $0.416 \pm 0.006$ & $0.388 \pm 0.006$ & $0.640 \pm 0.041$ & $0.627 \pm 0.043$ & 0.466 \\
\hline Err & $-51.54 \%$ & $-43.39 \%$ & $-45.81 \%$ & $-33.85 \%$ & $-10.66 \%$ & $-16.68 \%$ & $37.36 \%$ & $34.62 \%$ & \\
\hline 353 & $0.212 \pm 0.002$ & $0.239 \pm 0.002$ & $0.228 \pm 0.002$ & $0.267 \pm 0.003$ & $0.357 \pm 0.005$ & $0.331 \pm 0.005$ & $0.548 \pm 0.039$ & $0.548 \pm 0.039$ & 0.354 \\
\hline Err & $-40.01 \%$ & $-32.54 \%$ & $-35.60 \%$ & $-24.69 \%$ & $0.94 \%$ & $-6.47 \%$ & $54.66 \%$ & $54.65 \%$ & \\
\hline 363 & $0.205 \pm 0.002$ & $0.228 \pm 0.002$ & $0.217 \pm 0.002$ & $0.253 \pm 0.003$ & $0.334 \pm 0.005$ & $0.309 \pm 0.004$ & $0.512 \pm 0.037$ & $0.514 \pm 0.035$ & 0.314 \\
\hline Err & $-34.78 \%$ & $-27.42 \%$ & $-30.98 \%$ & $-19.45 \%$ & $6.30 \%$ & $-1.66 \%$ & $62.91 \%$ & $63.70 \%$ & \\
\hline
\end{tabular}


All the shear viscosities are combined in Figure 3-5 where the trends of the values versus the temperature can be easily recognized. At this time, no single model can reproduce the trend of the experimental data; instead, each model shows its own strength in a certain temperature range. When temperature is in the range from $298 \mathrm{~K}$ to $318 \mathrm{~K}$, five-site model could be the best choice; and if temperature is around $333 \mathrm{~K}$ four-site model is better. In particularly, four-site models (TIP4P-2005, TIP4P-Ew) precisely predict the shear viscosity at $353 \mathrm{~K}$ and $363 \mathrm{~K}$. It is interesting to notice that all of threesite model underestimate the shear viscosity by a certain relative error. However, this error is decreasing as temperature increase from $283 \mathrm{~K}$ to $363 \mathrm{~K}$, which hints that three site model may have best performance for hot water. Considering the computation cost, threesite model still could be an alternative choice when study hot water related problem. In addition, for lower temperature, none of these models can precisely estimate shear viscosity at lower temperature of $283 \mathrm{~K}$. Even the best one, five-site model reaches significant errors. 


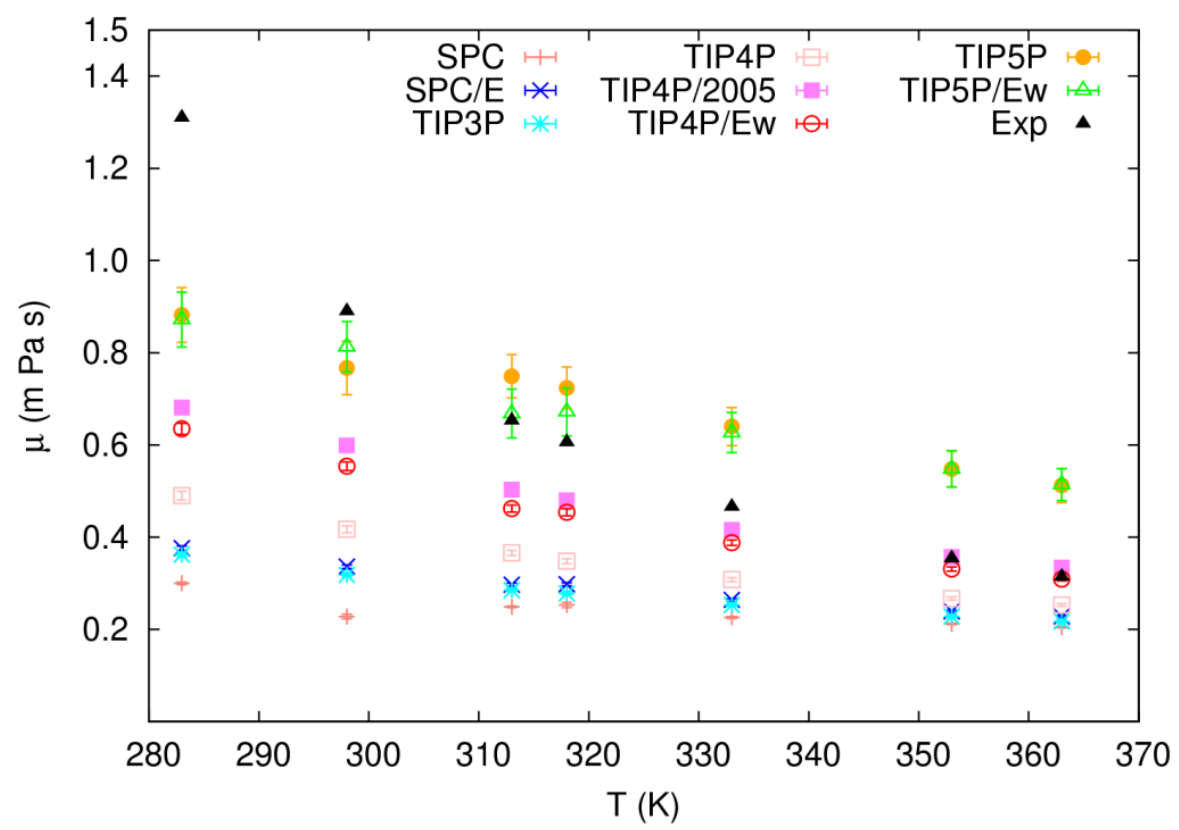

Figure 3-5 Shear viscosities trends predicted by eight rigid models

Table 3-4 summarizes the shear viscosities, uncertainties and relative error to experimental data for all cases reported in Figure 3-4. Uncertainties of each case are relatively small, and the maximum error of $7.92 \%$, comes from TIP5P-Ew at 313K. For lower temperature case $(283 \mathrm{~K})$, none of eight models can precisely reproduces the experiment value and the best result from TIP5P underestimate the viscosity by by $32.49 \%$. On the other hand, all of these eight rigid models accurately predict the decreasing trend of shear viscosity versus temperature. It can also be seen that TIP5P-Ew performed the best at $298 \mathrm{~K}, 313 \mathrm{~K}$, and $318 \mathrm{~K}$, with relative error of $-8.61 \%, 2.41 \%$, and $10.91 \%$, respectively; TIP4P-2005 has the minimum relative error when temperature is $333 \mathrm{~K}$ and $353 \mathrm{~K}$, with $-10.66 \%$ and $0.94 \%$ error; TIP4P-Ew shows the best agreement with experiment value at $363 \mathrm{~K}$ with only $-1.66 \%$ error. Based on current results, TIP4P does not show any advantage of predicting shear viscosity. 


\subsection{Conclusions}

In this work, thermal conductivities and shear viscosity of liquid water are computed with RNEMD approach and the results are compared among all results obtained from eight water rigid models. Linear temperature profiles can be well established for all rigid models and it is shown that five-site models can very precisely reproduce experimental value of thermal conductivities, while other models overestimate by tens of percent. For shear viscosity, none of all eight rigid models can reproduces all experiment values, but trends from each model accurately match that from the experiment. Five-site model can relatively accurately predict the real value of liquid water at lower temperature. Four-site model is a better choice of estimating shear viscosity at higher temperature. Therefore, the five-site models (TIP5P, TIP5P-Ew) are better choices if molecular dynamics simulation is used to study thermal emphasized problem for a water molecular system, like bubble nucleation in liquid water. For dynamic problem that involves water flow in micro- and nano- size systems, the five-site models (TIP5P, TIP5P-Ew) are also appropriate choices( in particular, for liquid water with lower temperature). When temperature is higher, TIP4P-2005 or TIP4P will be the best choice.

\section{Acknowledgement}

Support for this work by the U.S. National Science Foundation under grant number CBET- 1066917 is gratefully acknowledged. 


\section{Chapter 4 Nonequilibrium Molecule Dynamics Simulations of Nanobubble Growth and Annihilation in Liquid Water}

\subsection{Introduction}

Ranging from microdevices and macroscale hydraulic machinery developments, the increasing applications in industry require a deeper understanding on micro- or nanosized bubble behaviors, like bubble cavitations[45], nucleation[46], and stability[47]. Molecular dynamics (MD) simulation has been a powerful method to study microscale bubble behavior in many different situations. In aspect of cavitations and bubble nucleation, Park et al. systematically studied the hysteretic process of bubble collapse[48] with numerical simulations. $\mathrm{Wu}$ and Pan conducted MD simulation to study bubble nucleation rate in homogeneous liquid argon[49]. Tsuda et al. focused on bubble nucleation in liquid oxygen with different impurities[50]. Zhai et al. investigated bubble nucleation in supercritical Carbon Dioxide/Hexadecane solution, which is widely used in the plastic production industry[51]. In other aspects of bubble dynamics, Lugli and Zerbetto investigated nanobubble collapse in water associated with salts effect[52]. The stability study of helium bubble during high energy displacement was carried out in order to investigate the bubble damage in fusion reactor[53]. Insepov and Hassanein calculated the density and diffusion coefficient in liquid lithium which contains helium atoms during bubble formation by sampling data from MD simulation[54]. Nagayama et al. observed bubble formation in nano-channel with the emphasis on nucleation phenomenon in liquid argon[55]. For bubble formation and contraction simulation, Okumura and Ito investigated the processes in liquid argon by MD simulation[56], and their results 
indicated that the Rayleigh-Plesset equation is still valid for nano-sized bubble generated in liquid argon. Since most materials in the world are composed by polyatomic molecules (like water), where a more complicated force field should be accounted for, the bubble behavior in molecular liquid should be investigated.

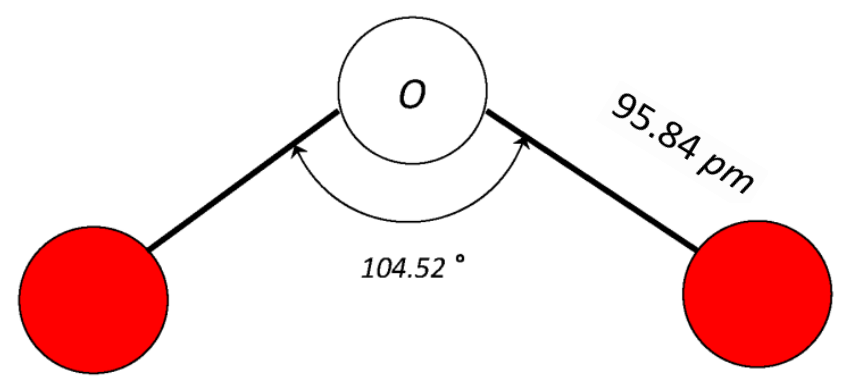

Figure 4-1 Water molecule illustration

The objective of this paper is to investigate nano-sized bubble growth and annihilation processes in liquid water and compare the results with that obtained from Rayleigh-Plesset equation. Sampling of the atomic movement data at the microscopic level during the entire process from the creation to the extinction will be discussed. The validities of the assumptions supporting the Rayleigh-Plesset equation will also be analyzed.

In order to investigate the dynamics of a bubble, reduced MD simulations is performed by applying the Lenard-Jones style potential. The characteristic dimensions of length, energy, and mass are chosen as the Lenard-Jones diameter $\sigma$, the minimum value of the potential $\varepsilon$, and the atom mass $m$. An asterisk $(*)$ will be used to represent the reduced quantities such as the reduced length $r^{*}=r / \sigma$, and the reduced temperature $T^{*}=$ $T k_{b} / \varepsilon$, the reduced pressure $P^{*}=P \sigma / \varepsilon$, the reduced mass density $\rho^{*}=\rho \sigma^{3} / m$, the reduced time $t^{*}=t\left(\varepsilon / m / \sigma^{2}\right)^{1 / 2}$, and the reduced charge $q^{*}=q\left(4 \pi \varepsilon_{0} \varepsilon \sigma\right)^{1 / 2}$. The hydrogen's 
properties in water molecule are chosen as characteristic dimensions during MD simulations for convenience of data analysis. All the results will be converted back to the familiar unit for discussion.

\subsection{Physical Models and Methods}

Nano bubble generation and its dynamics behavior is mainly affected by thermal conductivities and viscosities of the fluid. TIP3P did not show big difference in predicting both these water properties [24, 28], although TIP4P model is widely used for MD simulation. In addition, TIP3P has a better performance in describing intermolecular potential and controlling system pressure and it is also one of the popular water models in the rigid $T I P 3 P-C H A R M M[33,57,58]$ model that specified 3 interaction sites corresponding to 3 atoms in the structure. Thus, it is chosen to describe water molecule structure in this work. For the bond and angle model, the shake algorithm[33] is used to hold two $\mathrm{O}-\mathrm{H}$ bonds and $\mathrm{H}-\mathrm{O}-\mathrm{H}$ angle as rigid, while a harmonic bond model and a charmm angle model are applied respectively.

The interaction between two water molecules in TIP3P is represented as:

$$
E_{a b}=\sum_{i}^{\text {on } a \text { on } b} \frac{k_{C} q_{a_{i}} q_{b_{j}}}{r_{a_{i} b_{j}}}+\sum_{i}^{\text {on a on } b} \sum_{j} 4 \varepsilon_{a_{i} b_{j}}\left[\left(\frac{\sigma_{a_{i} b_{j}}}{r_{a_{i} b_{j}}}\right)^{12}-\left(\frac{\sigma_{a_{i} b_{j}}}{r_{a_{i} b_{j}}}\right)^{6}\right]
$$

where $k_{C}$ is the electrostatic constant that has a value of $332.06 \AA \cdot \mathrm{kcal} / \mathrm{mol}$ and $q_{\mathrm{i}}$ are the partial charges relative to the charge of the electron; $r_{i j}$ is the distance between charged sites; $\sigma_{a i}$ and $\sigma_{b j}$ are the Lenard-Jones parameters. The values of dimensionless parameters are given in Table 4-1. Arithmetic average is used to calculate the LJ potential between oxygen and hydrogen atom. 
Table 4-1 TIP3P water model parameters

\begin{tabular}{ccc}
\hline Items & REAL Units & LJ Units \\
\hline$m_{\mathrm{O}}{ }^{*}$ & $15.9994(\mathrm{~g} / \mathrm{mol})$ & 15.8724 \\
$m_{\mathrm{H}}$ & $1.008(\mathrm{~g} / \mathrm{mol})$ & -112.0 \\
$q_{\mathrm{O}}$ & $-0.834 \mathrm{e}$ & 56.0 \\
$q_{\mathrm{H}}$ & $0.417 \mathrm{e}$ & 3.30652 \\
$\varepsilon_{\mathrm{O}-\mathrm{O}}$ & $0.1521 \mathrm{Kcal} / \mathrm{mol}$ & 7.87617 \\
$\sigma_{\mathrm{O}-\mathrm{O}}$ & $3.1507 \AA$ & 1.0 \\
$\varepsilon^{*} \mathrm{H}-\mathrm{H}$ & $0.0460 \mathrm{Kcal} / \mathrm{mol}$ & 1.0 \\
$\sigma_{\mathrm{H}-\mathrm{H}}^{*}$ & $0.4000 \AA$ & 1565.32 \\
$K_{\mathrm{OH}}$ & $450 \mathrm{Kcal} / \mathrm{mol}^{\prime}\left(\mathrm{A}^{2}\right)$ & 2.39292 \\
$R_{\mathrm{OH}}^{0}$ & $0.9572 \AA$ & $1195.64 / \mathrm{rad}^{2}$ \\
$K_{\mathrm{HOH}}$ & $\left.55 \mathrm{Kcal} / \mathrm{mole}^{2} / \mathrm{rad}^{2}\right)$ & $104.52^{\circ}$ \\
$\theta_{\mathrm{HOH}}$ & $104.52^{\circ}$ & \\
\end{tabular}

* characteristic dimensions 
The particle-particle particle-mesh method $(P P P M)[32,59]$ is an accurate and computationally efficient method for calculating interactions in MD simulation, while its complexity is only $N \log (N)[32]$. Therefore, the long-range coulomb solver $P P P M$ was used to calculate the long-range force between the water molecules. During the computation, the PPPM solver maps water molecule's hydrogen and oxygen atoms charge in a 3D mesh, then uses 3D FFTs to solve Poisson's equation on each mesh. Finally, it interpolates from the grids to calculate electric field and the corresponding long-range Coulomb force on each charged-atom. Though, a careful choose of mesh size is required before applying PPPM approach in order to achieve a comparable precision of Ewald summation[19]. Here, number of 60 is selected to be mesh size in $x, y$, and $z$ direction, based on a pair of test cases for thermal conductivity $(278.15 \mathrm{~K})$ calculation which is carried out by employing Reverse Non-equilibrium Molecular Dynamics (RNEMD[27]). Long-range electrostatic force contribution in one case is computed with PPPM, while the other case is based on Ewald summation. It is found that when the size is set to be 60, the value from PPPM is $0.967 \pm 0.012 \mathrm{~W} / \mathrm{m} \cdot \mathrm{K}$, and the Ewald-based case is $0.954 \pm 0.013 \mathrm{~W} / \mathrm{m} \cdot \mathrm{K}$. Therefore, size of 60 can be reasonable choice. In addition, since the later results are based on statistically average, the error introduced by PPPM method is negligible.

Constant pressure MD simulations were parallel[36] done by performing time integration on Nose-Hoover style non-Hamiltonian equations of motion. All the positions and velocity of atoms are generated from NPH ensemble through Verlet algorithm with the following conditions. The cubic simulation box $(6 \mathrm{~nm} \times 6 \mathrm{~nm} \times 6 \mathrm{~nm})$ with periodic boundary condition has 7200 water molecules. For short range pair force, the widely used 
lj/charm/coul/long[60] styles is chosen to compute potential energy and corresponding force, which is contributed by van der Waals and Coulombic interactions force, by employing an additional switching function $S(r)$ that both ramps the energy and force smoothly to zero between an inner and outer cutoff:

$$
E(r)=\left\{\begin{aligned}
L J(r)+C(r), & r<r_{\text {in }} \\
S(r) L J(r)+S(r) C(r), & r_{\text {in }}<r<r_{\text {out }} \\
S(r) L J(r)+S(r) C(r), & r>r_{\text {out }}
\end{aligned}\right.
$$

where $r_{\text {in }}$ and $r_{\text {out }}$ are set to be $20 \sigma$ and $25 \sigma$ respectively. Other functions in eq. (2) are:

$$
\begin{gathered}
L J(r)=4 \varepsilon\left[\left(\frac{\sigma}{r}\right)^{12}-\left(\frac{\sigma}{r}\right)^{6}\right] \\
C(r)=f_{\text {damp }}(r) \frac{C q_{i} q_{j}}{\varepsilon r} \\
S(r)=\frac{\left[r_{\text {out }}^{2}-r^{2}\right]^{2}\left[r_{\text {out }}^{2}+2 r^{2}-3 r_{\text {in }}^{2}\right]^{2}}{\left[r_{\text {out }}^{2}-r_{\text {in }}^{2}\right]^{3}}
\end{gathered}
$$

where $\sigma$ and $\varepsilon$ are determined by atom type, and $f_{\text {damp }}(r)$ in eq. (4) is determined not only by distance between atoms but also related to $G$-Ewald parameter which will be adjusted through mesh size and desired precision.

A harmonic bond model[60] is used in computing the bond force between oxygen and hydrogen atoms, and potential energy contributed is calculated:

$$
E=K\left(r-r_{0}\right)^{2}
$$

The charmm angle style[60], which considers the potential energy contribution from the alteration of bond angle from balanced $\theta_{0}$ with an additional Urey-Bradley term, is chosen as the angle model, i.e.,

$$
E=K\left(\theta-\theta_{0}\right)^{2}+K_{U B}\left(r-r_{U B}\right)^{2}
$$

where $K, \theta_{0}, K_{u b}$, and $r_{u b}$ are coefficients defined for each angle type.

The system pressure, after 20,000 time steps equilibrated through Langevin thermostat, is 
well controlled to be 0 (1 bar), while initial temperature is equilibrated to $12.0(278.15 \mathrm{~K})$. The time step is $0.005 t^{*}(0.46 f s)$. Then 80 molecules near the center of the simulation box are selected, and instantaneously heated to $132(3059.65 K)$ by velocity rescaling. The temperature in the domain including the heated atoms is much higher than boiling point which is $16.11(373.5 K)$ at one atmosphere. During the latter 11,000 time steps, the atomic movement of the system is recorded until the bubble extinct.

The volume and the radius of the bubble are estimated by the following method described below. First, all sides of the simulation cell were divided into 10 sections so the simulation box is divided into 1000 small cubes. The side length $\Delta L^{*}$ of the small cube fluctuated between $14.87(5.95 \AA)$ and $14.95(5.98 \AA)$ during the MD simulations. The average density is calculated for each small cube over 100 time steps, which is much smaller than the time scale of the bubble dynamics (11,000 steps) and much larger than that of the atomic dynamics ( $\sim 20$ steps). The vapor region is recognized as the one in which the average density in the small cube is less than the critical density $\rho_{c}{ }^{*}=6.83 \times 10^{-4}$ $\left(320 \mathrm{~kg} / \mathrm{m}^{3}\right)[37]$ and the region of liquid is the one where the average density is greater than $\rho_{c}{ }^{*}$. The bubble volume was calculated as the sum of the volumes of the vapor regions. Since the bubble is fairly spherical shape, the effective bubble radius $R^{*}$ is estimated from bubble volume $V_{b u b}{ }^{*}$ using the following equation:

$$
R^{*}=\left(\frac{3}{4 \pi} V_{b u b}^{*}\right)^{1 / 3}
$$

In order to improve the statistical accuracies, $15 \mathrm{MD}$ simulations with different initial molecule configuration are performed. The error bar is calculated by the standard deviation of $R^{*}$ from the 15 cases.

In macroscopic scale, a hydrodynamic equation, namely Rayleigh-Plesset equation, 
which is applicable for an incompressible and inviscid fluid, can be used to describe expansion and contraction of a bubble:

$$
R^{*} \ddot{R}^{*}+\frac{3}{2} \dot{R}^{*}=\frac{1}{\rho_{l}^{*}}\left[P^{*}\left(R^{*}\right)-P_{\infty}^{*}-\frac{2 S^{*}}{R^{*}}\right]
$$

where $\mathrm{P}^{*}\left(\mathrm{R}^{*}\right)$ is the pressure at the bubble surface and $\mathrm{P}_{\infty}{ }^{*}$ is the pressure far from the bubble in the liquid region, and $\mathrm{S}^{*}$ is the reduced surface tension of the bubble. It is difficult to precisely determine $\mathrm{P}^{*}\left(\mathrm{R}^{*}\right)$ since the radius of the generated bubble is only a few times of the molecular diameter.

In this work, the molecules constituting the bubble surface are used to estimate the surface pressure of the water bubble:

$$
P^{*}\left(R^{*}\right)=\frac{\rho_{c}^{*}}{3} \frac{1}{N_{s}-N_{c}}\left\{\sum_{i=1}^{N_{s}} m_{i}^{*} \dot{\boldsymbol{r}}_{i}^{2}+\left[\sum_{n=1}^{N_{s}}\left(\boldsymbol{r}_{i} \cdot \boldsymbol{f}_{i}\right)\right]_{b, a, s, k}\right\}
$$

where Ns is the molecule number within Router of the MD simulation cell, as shown in Figure 4-2, and it can be simply calculated by:

$$
N_{s}=V^{*} \rho_{l}^{*}
$$

where the density at edge of the effective bubble is assumed to be liquid density, and $\mathrm{V}^{*}$ is the volume of the spherical shell that can be estimated by:

$$
V^{*}=4 \pi\left[R_{\text {outer }}^{*}{ }^{3}-\left(R_{\text {outer }}^{*}-d_{m}^{*}\right)^{3}\right] / 3
$$

$N_{c}$ in eq. (10) is the molecule number within the inner spherical region, as shown in Figure 4-2, and their contribution to pressure will not be accounted for. Label $b, a, s, k$, in eq. (10) represents pair interaction, bond, angle, interactions, internal constrain force to atoms from shake algorithm and long range force contribution respectively. And $d^{*} m$ is the diameter of a single water molecule. 


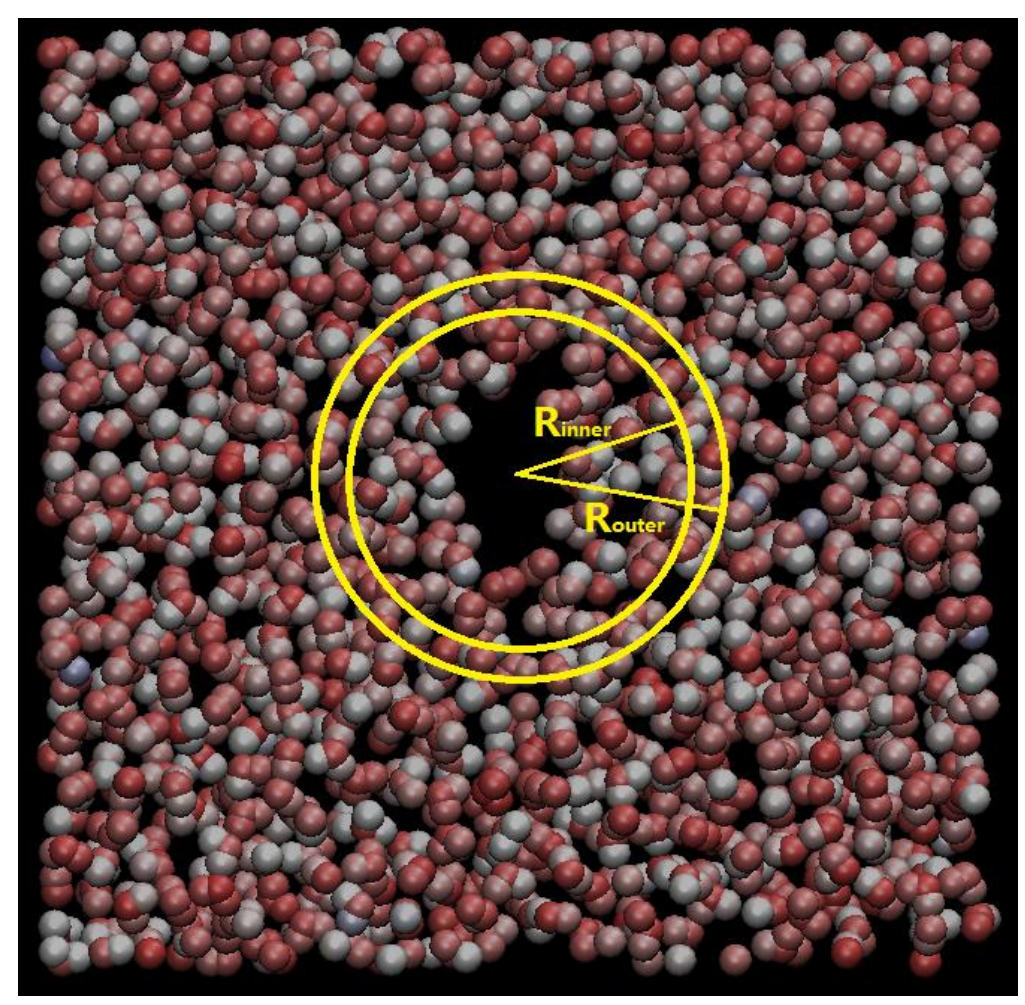

Figure 4-2 Molecule number estimation in one molecule thinkness layer

\subsection{Results and Discussions}

All MD simulations in this paper are performed using LAMMPS, which is an open source software developed by the Sandia National Laboratories. Figure 4-3 shows snapshots of the bubble formation and annihilation using the VMD[61] software. These snapshots show the molecules that are in a $10 \%$ slab of the simulation box in the depth direction. It can be seen that the heated molecules scatter the neighboring non-heated molecules until a bubble is created and then the bubble is cooled and compressed by the surrounding sub-cooled liquid later. Physically, the molecules in the center, which is colored as white, are rescaled to the desired temperature at time 0 , and heat is transferred to the surrounding molecules along the temperature gradient by interaction between the 
hot molecules and the cold ones. As a result, a bubble is generated as shown during the period from time $0.368 p s$ to $2.162 \mathrm{ps}$. It is worth to mention a $0.368 \mathrm{ps}$ time lag exists before a clear bubble come out. After time $2.162 \mathrm{ps}$, the bubble compressed by those cold surrounding liquid molecules, then it turns to be smaller and smaller, finally it diminishes. 


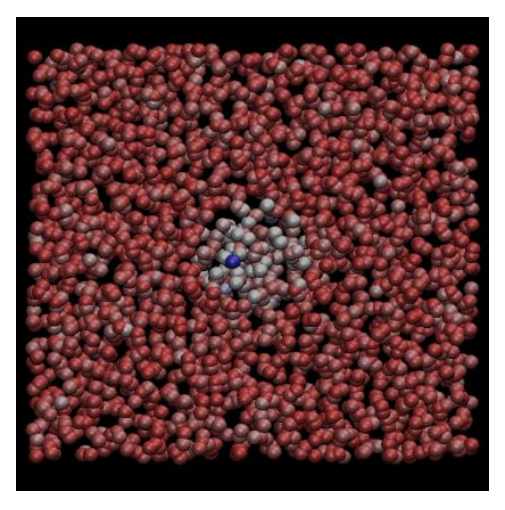

Time 0 ps

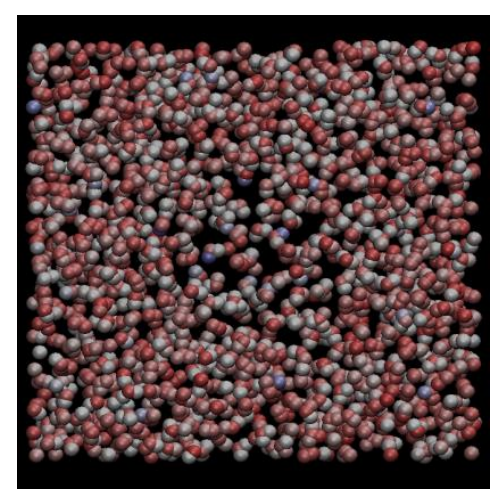

Time 0.874 ps

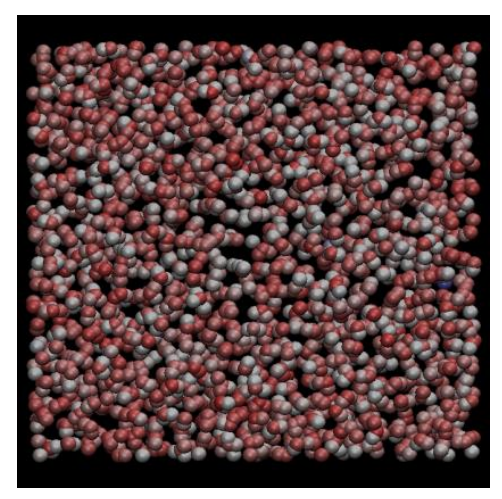

Time 3.082 ps

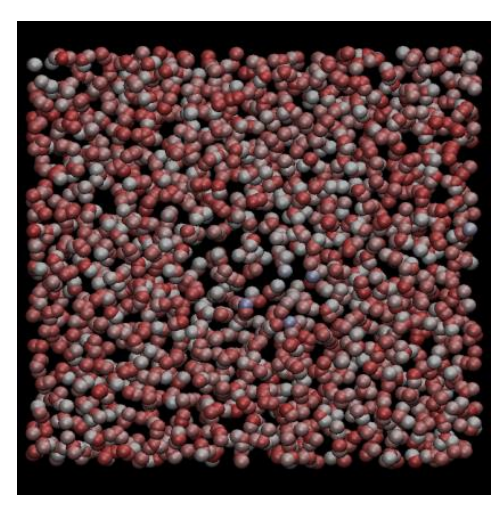

Time 0.368 ps

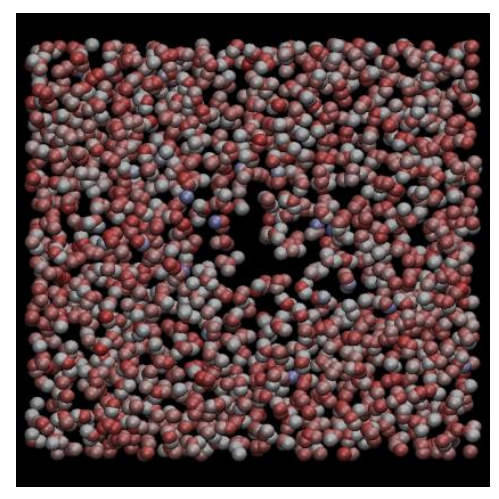

Time 1.242 ps

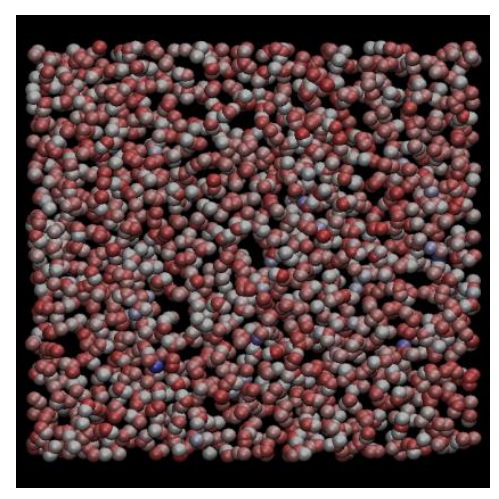

Time 3.956 ps

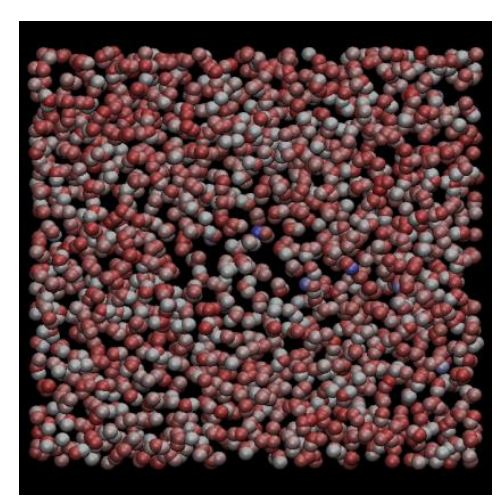

Time 0.644 ps

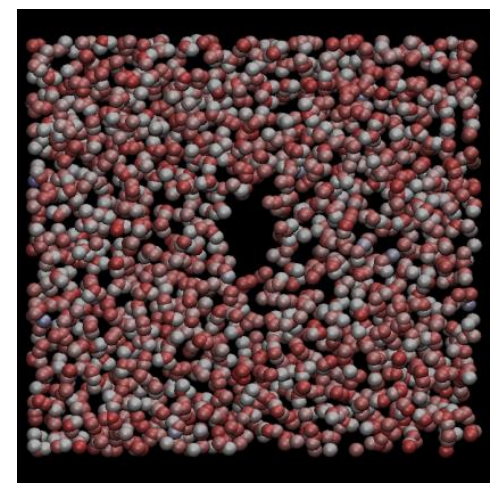

Time 2.162 ps

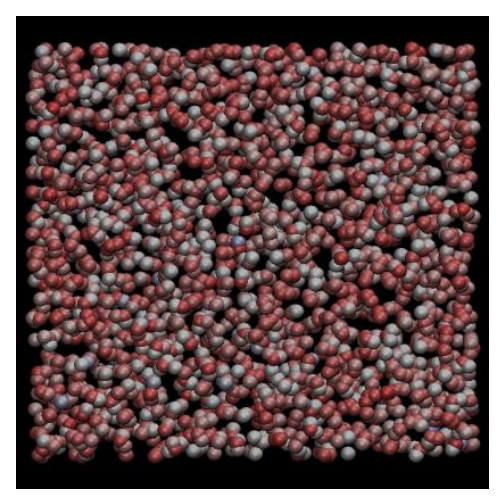

Time 5.06 ps

Figure 4-3 Snapshots of the bubble during the period from 0 ps to $5.06 \mathrm{ps}$ 
Correspondingly, a density profile within the $10 \%$ slab of simulation box in depth direction is computed by uniformly chopping the box into 1000 small cubes whose density is obtained by averaging over all 15 cases. 


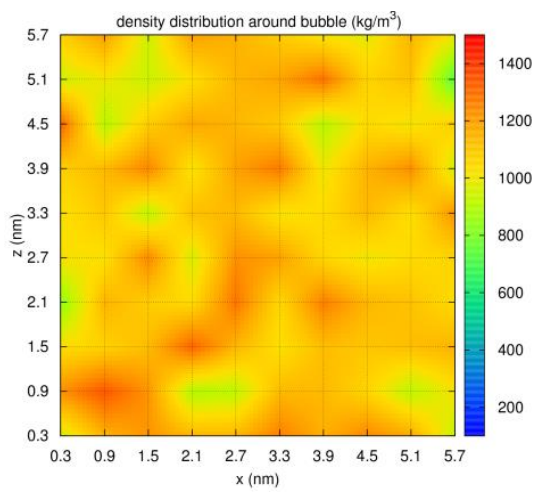

Time 0 ps

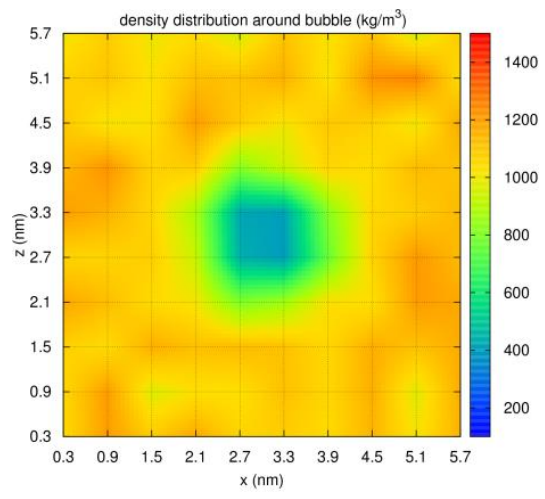

Time 0.874 ps

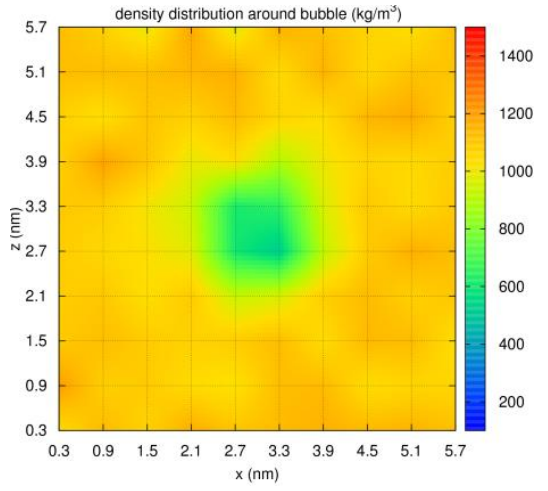

Time $3.082 \mathrm{ps}$

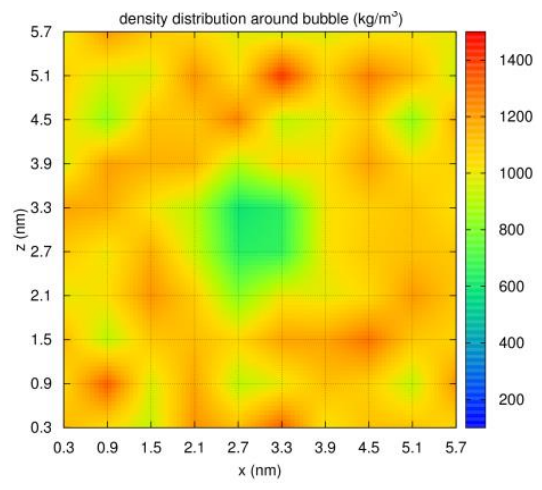

Time 0.368 ps

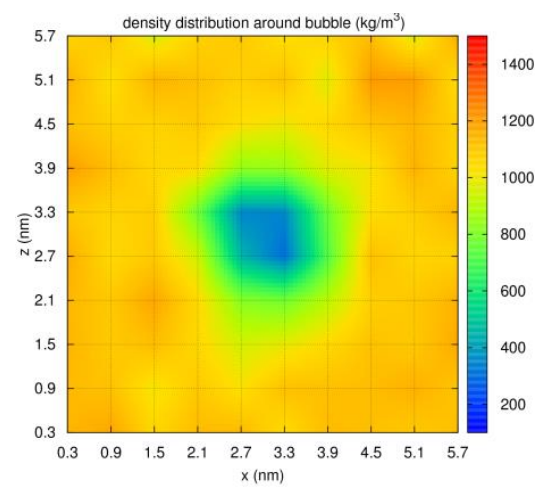

Time $1.242 \mathrm{ps}$

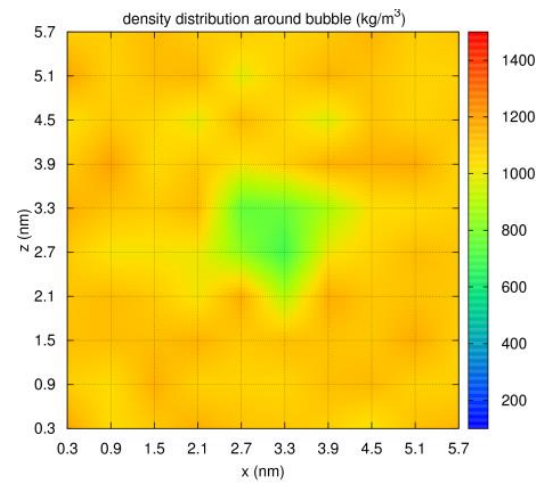

Time $3.956 \mathrm{ps}$

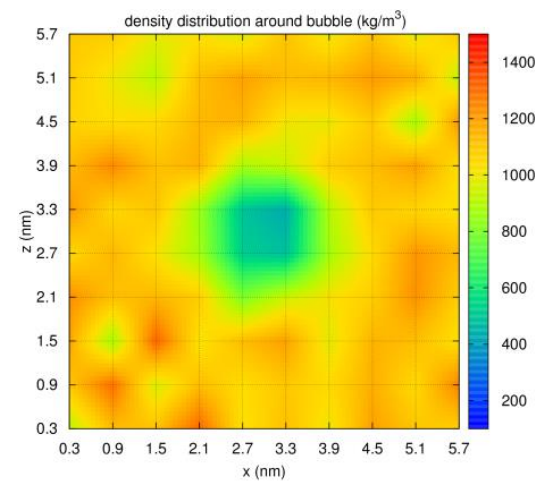

Time 0.644 ps

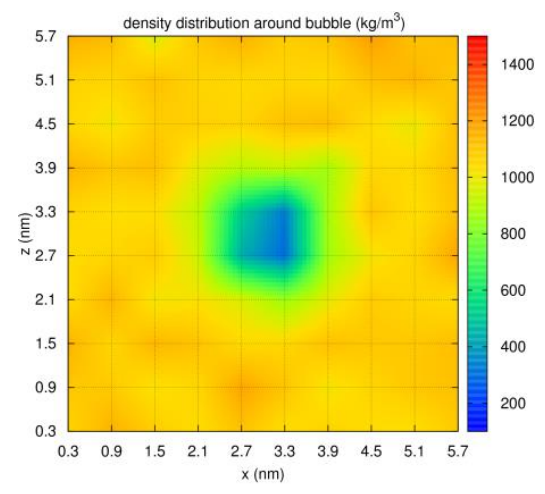

Time $2.162 \mathrm{ps}$

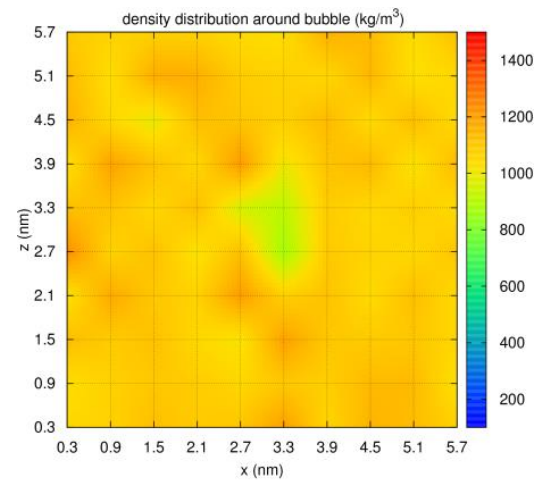

Time $5.06 \mathrm{ps}$

Figure 4-4 Density profile from 0 ps to 5.06 ps 
The range of legend in Figure 4-5 is fixed from $100 \mathrm{~kg} / \mathrm{m}^{3}$ to $1500 \mathrm{~kg} / \mathrm{m}^{3}$ in order to easily recognize the bubble shape within the box and clearly see the density distribution variation. At time $0 \mathrm{ps}$, density is not uniformly distributed, instead, fluctuating around $1000 \mathrm{~kg} / \mathrm{m}^{3}$, which is the density of liquid water at 1 bar pressure. If look through these series figures, it can be realized that bubble expands from $0 p s$ to $2.162 \mathrm{ps}$, and begin its annihilation during the later period. The density finally comes to be $1000 \mathrm{~kg} / \mathrm{m}^{3}$ again at $5.06 \mathrm{ps}$. 


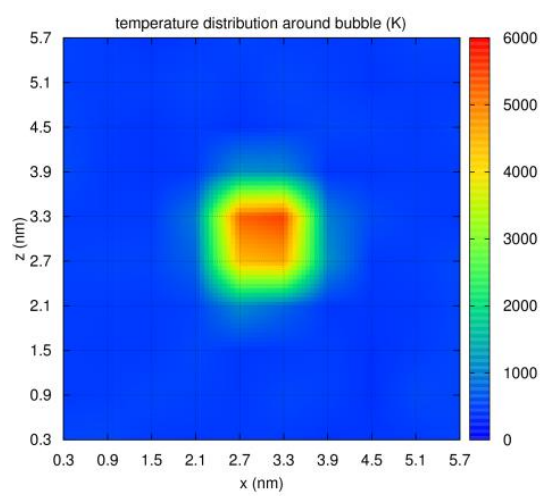

Time 0 ps

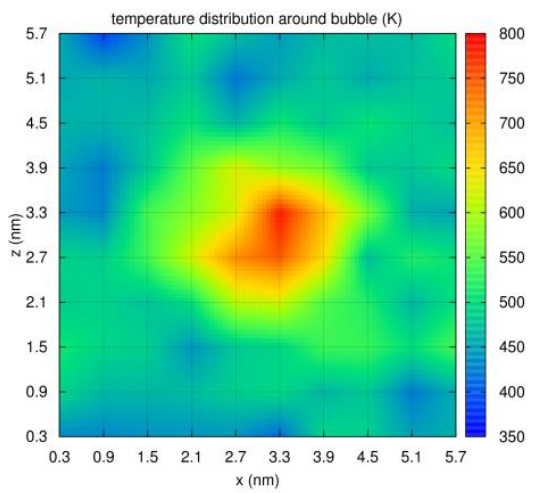

Time 0.874 ps

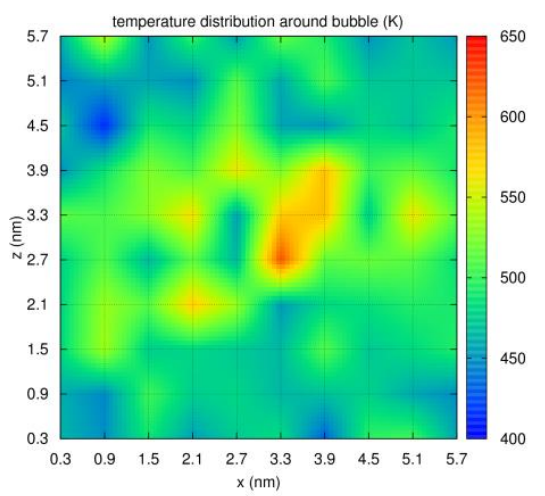

Time 3.082 ps

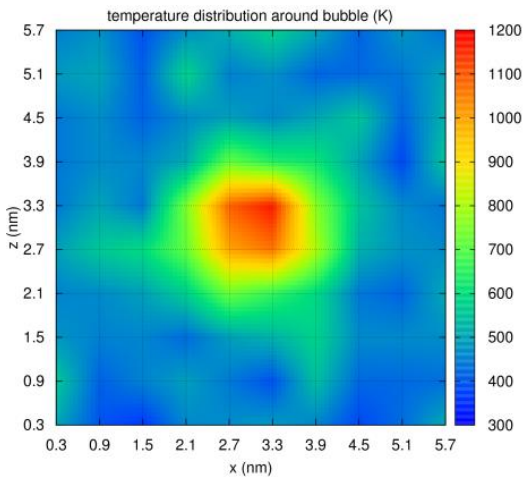

Time 0.368 ps

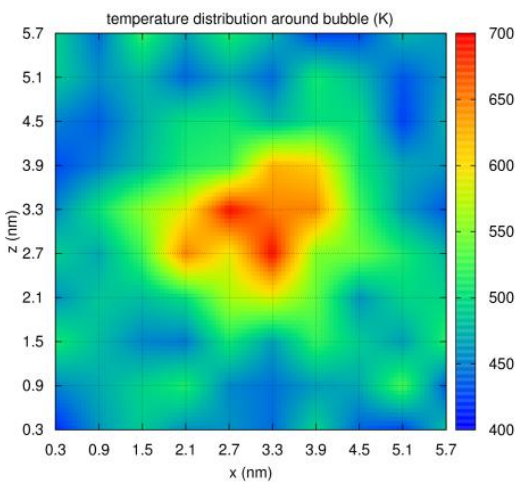

Time 1.242 ps

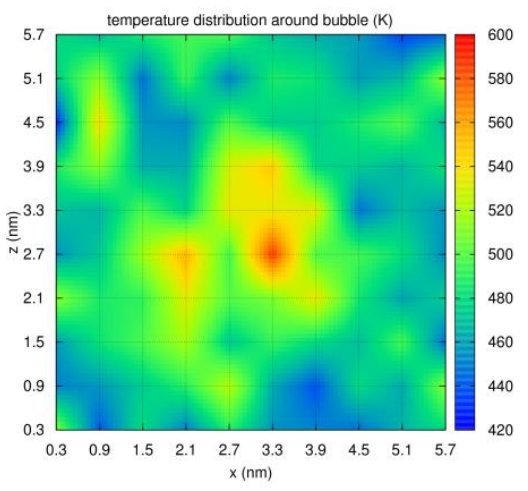

Time 3.956 ps

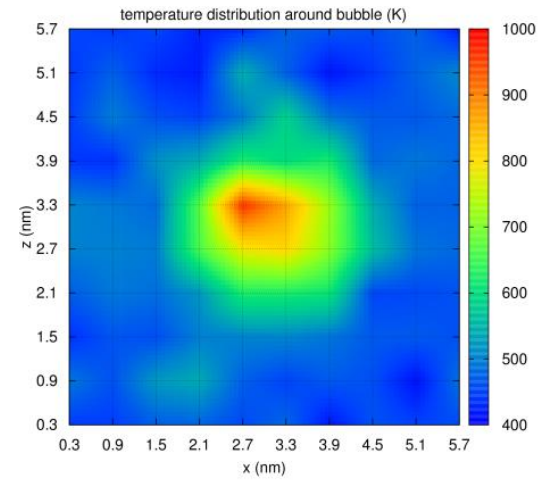

Time $0.644 \mathrm{ps}$

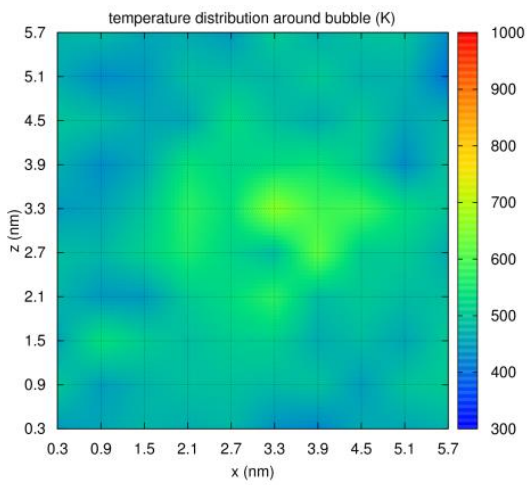

Time 2.162 ps

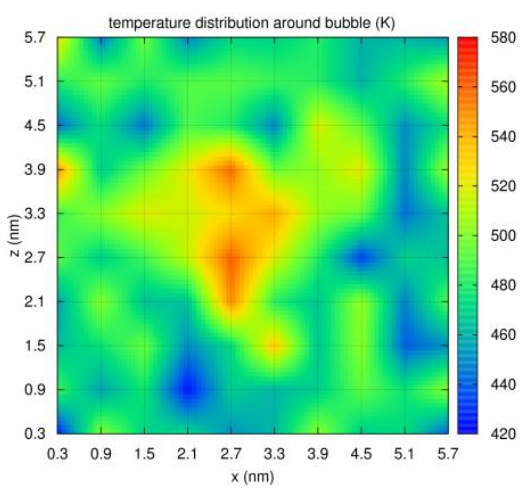

Time $5.06 \mathrm{ps}$

Figure 4-5 Temperature profile from $0 \mathrm{ps}$ to $5.06 \mathrm{ps}$ 
With the purpose of showing the heat spreading around the bubble, temperature distribution in the 1000 cubes are also computed and given in Figure 4-5.

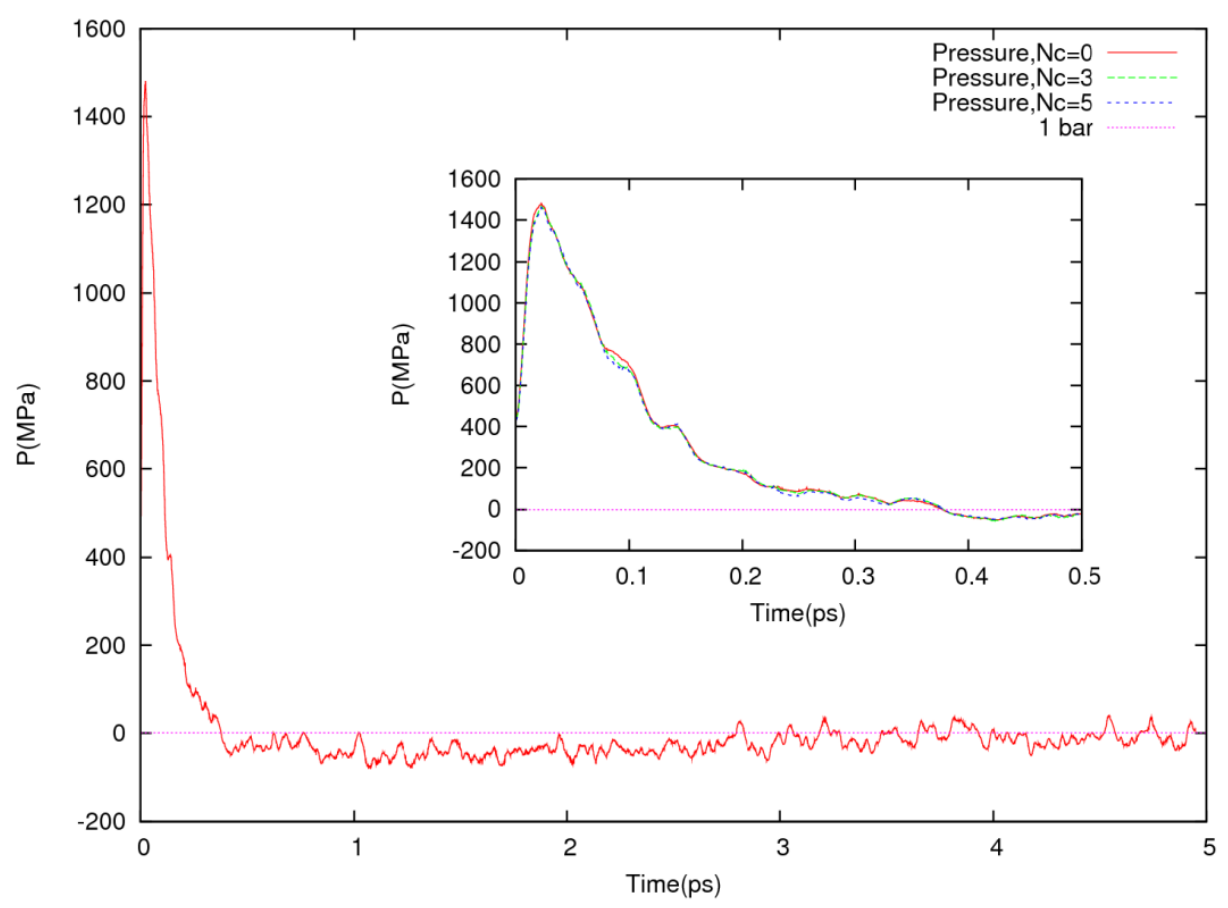

Figure 4-6 Pressure at the surface of the bubble

Since the temperature varies within a very large range, from $300 \mathrm{~K}$ to $6000 \mathrm{~K}$, the range of legend is not unified in order to easily see the temperature differences. It should be mentioned that the highest temperature is around $6000 \mathrm{~K}$ which is much higher than the desired temperature of heating $(3059.65 \mathrm{~K})$ at time 0 , because $3059.65 \mathrm{~K}$ is a statistical average temperature, it is possible that some atoms reaches to very high value. At the beginning, it can be seen that the region around heated zone is almost uniformly has same temperature, but within a very short period $(0.368 \mathrm{ps})$, a clear temperature difference in the distribution around the hot bubble can be recognized due to the fast heat spreading to surrounding cool liquid. It is interesting to notice that at time $2.162 \mathrm{ps}$, when the bubble fully expanded, the temperature seems relatively uniformly distributed, but during the 
latter period from $3.082 \mathrm{ps}$ to $5.06 \mathrm{ps}$, an obvious difference in temperature distribution comes out due to fluctuation of wave in water flow. But the average temperature has an apparent decline.

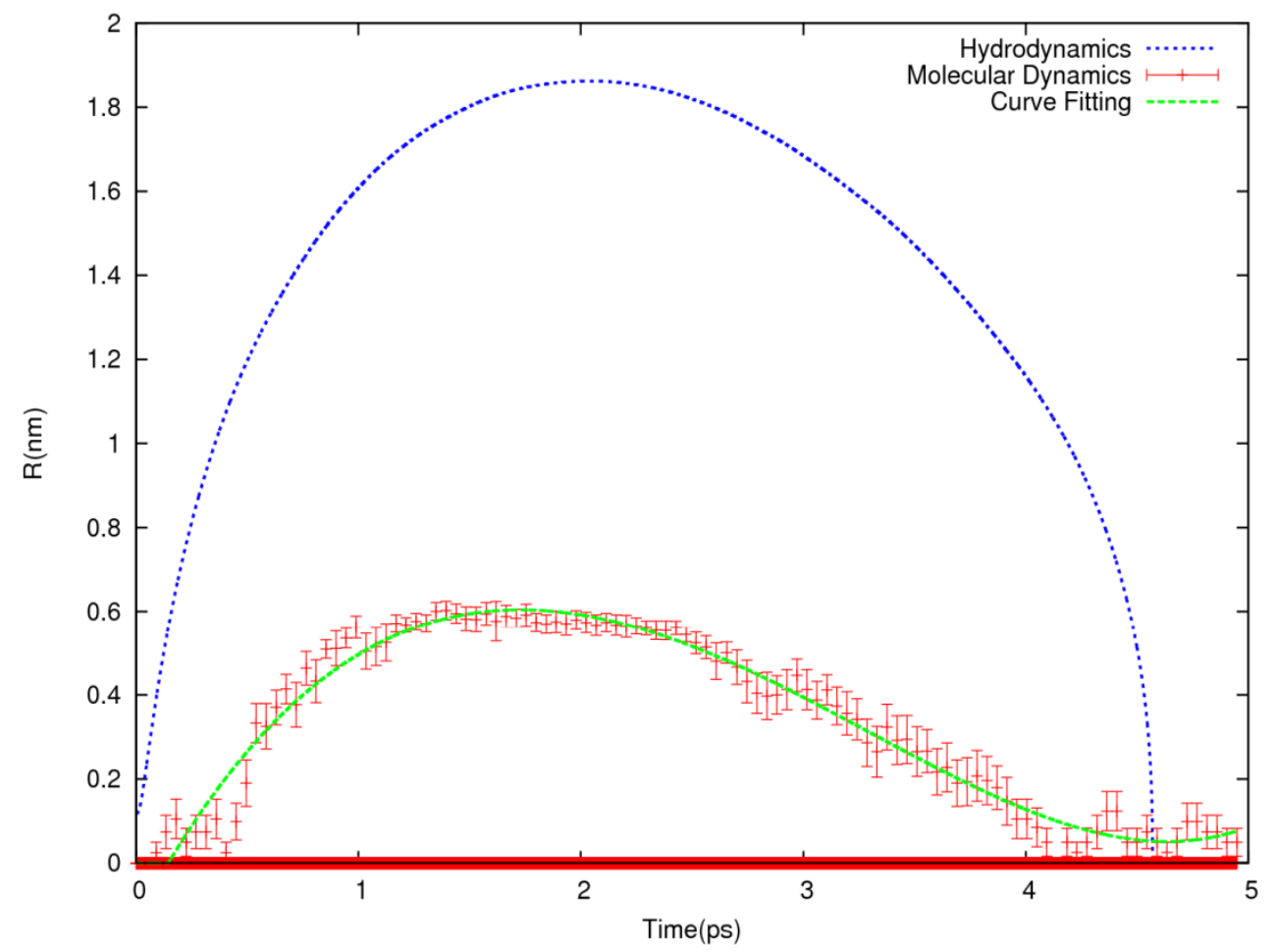

Figure 4-7 Bubble radius variation in water (S refer to Sample Point)

Figure 4-6 shows the calculated pressure at the surface of the generated bubble. The result from $N_{c}=0,3,5$ does not have a great difference which means the pressure estimated from $N_{s}=20$ is reliable. It can be seen that the pressure value immediately reaches to $1450 \mathrm{Mpa}$ in $0.025 \mathrm{ps}$, and then decrease relatively slowly to atmosphere pressure during the following period from $0.025 \mathrm{ps}$ to $0.375 \mathrm{ps}$. The fluctuation from $0.375 p s$ to $5 p s$ indicates that the pressure fall down to be the system pressure of 1 atm again. It is interesting to notice, the pressure is dramatically increase from $0.1 \mathrm{Mpa}$ to $1450 \mathrm{Mpa}$ within very short time, $0.025 \mathrm{ps}$, after 1 step heated. Indeed, if take a 
comparison between Figure 4-6 and the bubble behavior history at Figure 4-4 (or Figure 4-5) at time scale, it can be found that the bubble is still expanding even the pressure comes down to the atmosphere pressure. Later, this pressure history will be used to predict the bubble radius evolution through eq. (9).

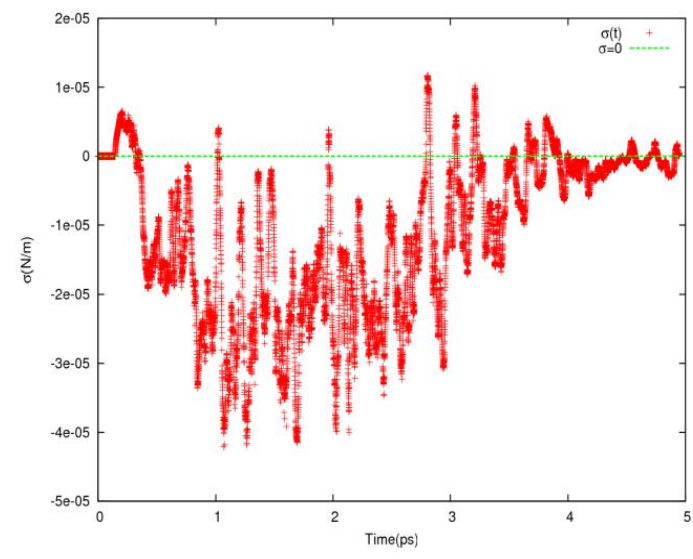

(a)

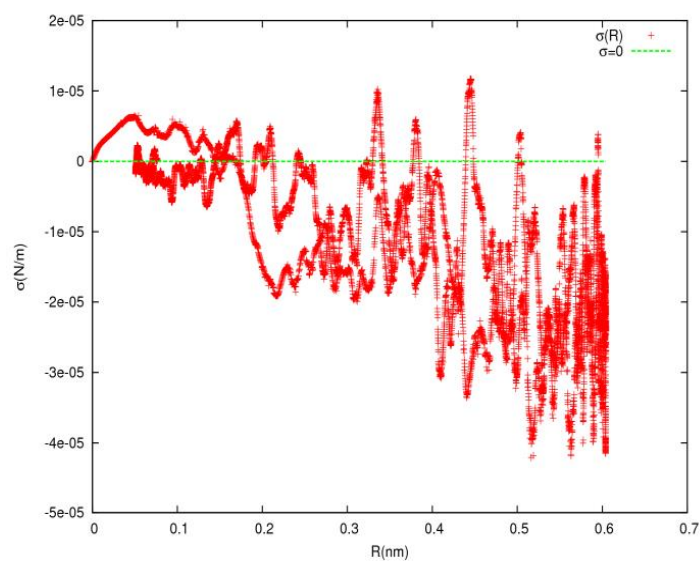

(b)

Figure 4-8 Surface tension profiles obtained by assuming the validity of the hydrodynamics equation

Figure 4-7 shows the time history of the calculated bubble radius. The "Hydrodynamics" line is obtained by using the hydrodynamic model (Rayleigh-Plesset equation). It is observed that if a nonzero surface tension is used, the curve span in time cannot cover that recorded from MD simulation. Indeed, even a decent piecewise function of surface tension $(S(R))$ is used in hydrodynamic model; radius of bubble will fall to zero within a much shorter time than that obtained from MD. Therefore, in order to cover the whole period of bubble behavior, the surface tension is set to be 0 . The error bar line (Molecular Dynamics) represents the statistical result obtained from the $15 \mathrm{MD}$ cases; the "Curve Fitting" line is a fitted line based on MD simulation results, which will 
be discussed later. It can be seen that the bubble immediately grows after the heating and reaches to the maximum radius during the $4.6 \mathrm{ps}$ long period. In other words, the bubble has the maximum radius $0.6 \mathrm{~nm}$ at $t=1.69 \mathrm{ps}$ and disappears at $t=4.6 \mathrm{ps}$. It is worth to mention that the lower value fluctuation of radius after $t=4.6 \mathrm{ps}$ comes from the density fluctuations in the liquid, which means that the bubble size is viewed as zero during the period after $4.6 \mathrm{ps}$. Thus the essentially important $R$ value is only the ones shown before $t$ $=4.6 \mathrm{ps}$. The radius predicted by Rayleigh-Plesset equation is obviously larger than that from MD simulation during the entire process, roughly by two times. Various possible surface tension functions are tested in order to match the radius variation obtained from MD simulation.

However, as reported above, a possible surface tension function is not found. Here, the existence of such a surface tension function is further studied by assuming the Rayleigh-Plesset equation is still valid in the liquid water. First, the bubble radius time history function $R(t)$ is obtained based on the radii from MD simulations by curve fitting, and the following polynomial fitted equation with $R^{2}=99.97 \%$ is obtained:

$$
\begin{aligned}
R(t)=1.053 \times 10^{-7} t^{5}-1.289 \times 10^{-5} t^{4}+1.272 \times 10^{-3} t^{3}-8.778 \times 10^{-2} t^{2}+2.258 t \\
-3.415
\end{aligned}
$$

By substituting the fitted equation of $R(t)$ into Rayleigh-Plesset equation, both of $S(t)$ and $S(R)$ are obtained and plotted in Figure 4-8. Figure 4-8(a) shows the surface tension variation with time. Figure 4-8(b) gives that the surface tension varies with corresponding radius. Both of the result shows a non-physical surface tension function (non-positive surface tension) should be applied to reproduce MD results If take a close look at Figure 4-8(a), during the time period from 0 to $\sim 0.1 \mathrm{ps}$, a zero surface tension should be applied, and then a positive surface tension will be used from $\sim 0.1 \mathrm{ps}$ to $\sim 0.3 \mathrm{ps}$, then a long non- 
positive value have to be used to drag the expanding bubble down. From Figure 4-8(b), it can be seen there are a pair of $\sigma$ corresponding to one radius, due to expanding and compression procession. The surface tension $\sigma$ corresponding to one radius value are not exactly overlapped, but has a similar trends, so it can be considered this method to find possible $S(R)$ is reasonable. In conclusion, Figure 4-8 indicate that a reasonable surface tension function $S(R)$, which can reproduce the results of MD simulation, does not exist at all.

Viewing from physics aspect, the surface tension in water bubble is contributed by a more complicated force field than monatomic argon liquid. In fact, the atomic structure of a water molecule consists of two positive charged hydrogen atoms joined to one negative charged oxygen atom with a bond angle of $104.52^{\circ}$. This unique way causes one side of the molecule to have a positive charge while the area in the opposite direction to have a negative charges[62]. Therefore, water molecules are attracted to each other, and form strong molecule bonds as a result from polarity of charge within one molecule. If comparing argon liquid and water liquid, the main difference is that water molecule involve electrostatic force, bond and angle interaction. In other words, it has a more complicated force field. Considering the fact that there are many other possible water models existing in literature field (3-sites, 4-site, 5-site, rigid, flexible, etc.), the surface tension contributed by this complicated force field should be further investigated for liquid water in microscale in order to further verify the hydrodynamic equation. Thus, based on the current results on TIP3P model, it can only be concluded that prediction water bubble dynamics with Rayleigh-Plesset equation cannot match with that from MD simulation, which is different from that for growth and annihilation of nano-sized bubble 
in the monatomic liquid argon. It should also be pointed out that the current form of Rayleigh-Plesset equation is derived by ignoring the viscosity and density fluctuation of liquid. However, viscosity may not be a possible reason to cause the invalid of hydrodynamic model, because the temperature during the period is sufficiently high to ignore the viscosity. Even when the bubble disappears, lowest temperature within the domain is $420 \mathrm{~K}$. Compressibility may also not be a reason leading to the invalidity, the volume fluctuation of the simulation box is small, and density change during the process is also very small.

\subsection{Conclusions}

Nanobubble formation and annihilation processes are simulated in polyatomic molecule liquid (water) through non-equilibrium molecular dynamic simulation. After equilibration for 20,000 time steps, 80 molecules near the center of the simulation box are selected, and instantaneously heated to $3059.65 \mathrm{~K}$ by velocity rescaling. During the latter 11,000 time steps, the atomic movement of the system is observed until the bubble extinct. In order to improve the statistical accuracies, $15 \mathrm{MD}$ simulations with different initial molecule configuration are performed. Pressure at the surface of the nano-sized bubble immediately increases to $1450 \mathrm{Mpa}$ from $0.1 \mathrm{Mpa}$ within $0.025 \mathrm{ps}$, and later decay to be 1 bar within $0.4 \mathrm{ps}$. Bubble fully expands to $\mathrm{R}=0.6 \mathrm{~nm}$ at $1.69 \mathrm{ps}$, and disappeared at $4.6 \mathrm{ps}$. A time lag, which has a time length of about $0.368 \mathrm{ps}$, is observed before a clear bubble come out after heating the molecules. Density profile within the heated simulation box is recorded to shown the evolution of density distribution, and a clear bubble is captured. The heat spreading process around the hot bubble is also observed. The results, based on TIP3P model, show that the hydrodynamic model based on Rayleigh-Plesset 
equation is not valid in predicting nano-sized bubble growth and annihilation in liquid water. Since difference force field can lead to different surface tension on the bubble, and considering the existence of many possible water models, further investigation on validity of Rayleigh-Plesset equation should be carried out.

\section{Acknowledgement}

Support for this work by the U.S. National Science Foundation under grant number CBET- 1066917 is gratefully acknowledged. 


\section{Chapter 5 Molecular Dynamic Study on Contact Angle of Water Droplet on a Single-Wall Carbon Nanotube (SWCNT) Plate}

\subsection{Introduction}

Since the rediscovery of carbon nanotube in 1991[63], it has attracted intensive attentions of studies due to its extraordinary mechanical and thermal properties[64]. Many potential applications of carbon nanotubes have been addressed[64-67] and some of them could occur at the interface between liquid water and CNTs, like nanofluidic device AFM tips[68, 69], single molecule detector[69], and electronic cooling fins[66]. Thus, many academic efforts have been carried out to investigate the wetting mechanism of water droplets on graphitic materials[70, 71]. One of the hottest subjects is wettability of CNT-based material. Journet et al.[72] has studied how fluid pressure affects the contact angle experimentally. Men et al.[73] reported a fabrication of superhydrophobic surface based on multi-walled carbon nanotubes. Werder et al.[74] investigated contact angles of a water droplets confined in different size carbon nanotubes by MD simulation, and their results indicates that nonwetting behavior of the pristine CNT occurred at room temperature; a 2008 review paper summarized that many MD studies were carried out on confined water droplet in different chiral CNTs with different water models[75]. In addition, hydrophobicity is another attractive feature of CNT forests, and it has been an important topic of CNT-related study due to its great importance to fundamental research and promising industrial applications. Tzeng et al.[76] have investigated the hydration properties of carbon nanotubes and their effect on electrical and biosensor application. 
Various CNT related superhydrophobic surface stand out. Huang et al.[77] created a stable superhydrophobic carbon nanotubes coated surface by coating it with a thin $\mathrm{ZnO}$ film. Li et al.[78] obtained a super-hydrophobic surface of bulk carbon nanotubes compacted by spark plasma sintering after modification with polytetrofluorethylene. Recently, Aria and Gharib[79] released a video of a bouncing water droplet on a carbon nanotube array to demonstrate its superhydrophobicity. The mechanism of interaction between water and $\mathrm{CNT}$ is rarely studied. In much numerical studies, quadrupole interaction which has been demonstrated as very important by quantum mechanical calculation[80] in liquid and gas phase of water was barely considered. In addition, most of CNT used in previous numeric studies are open-end type, while CNTs with capped end, which has a more reasonable chemical structure, are rarely applied due to difficulty of modeling. In this paper, the wettability of CNT with different separation distances will be studied to obtain a critical separation distance. As the second part of this work, a capped-end CNT plate will be created and a sphere-like liquid water droplet will be placed on top for the initial configuration of MD simulation. The effects of quadrupole potential and separation distance on wettability and contact angle will be analyzed.

\subsection{Physical Models and Methods}

Both water and carbon nanotube models should be addressed. There are many different water models available in the literature. A three-site water model (TIP3P[43]) is selected to model liquid water in the consideration of relatively high accuracy in description of liquid water's dynamic property[32] and affordable computational cost. The well-accepted Lenard Jones-like potential which consists of the contributions from electrostatic, dispersion and repulsive forces, as expressed below, is used to describe 
intermolecular interaction.

$$
U_{a b}=\sum_{i}^{a} \sum_{j}^{b} \frac{k_{C} q_{a_{i}} q_{b_{j}}}{r_{a_{i} b_{j}}}+\sum_{i}^{a} \sum_{j}^{b} 4 \varepsilon_{a_{i} b_{j}}\left[\left(\frac{\sigma_{a_{i} b_{j}}}{r_{a_{i} b_{j}}}\right)^{12}-\left(\frac{\sigma_{a_{i} b_{j}}}{r_{a_{i} b_{j}}}\right)^{6}\right]
$$

where, $a$ and $b$ denotes two different molecules, subscript $i$ and $j$ represent atom of hydrogen or oxygen in one individual TIP3P molecule, and $k_{\mathrm{c}}$ is electrostatic constant. The long-range columbic contribution to the MD system is computed with Ewald Summation[33, 81]. In TIP3P, only the interaction between atoms of oxygen is accounted for. The bonded interaction within water molecules are considered as rigid that is hold with the SHAKE[33] algorithm, so that larger time step can be employed.

Table 5-1 potential function parameters of water related interaction

\begin{tabular}{ccc}
\hline Parameters & Values & Units \\
\hline$\varepsilon_{O O}$ & 0.004386 & $\mathrm{eV}$ \\
$\sigma_{O O}$ & 3.188 & $\AA$ \\
$q_{\mathrm{H}}$ & 0.417 & $\mathrm{e}$ \\
$q_{\mathrm{O}}$ & -0.834 & $\mathrm{e}$ \\
\hline
\end{tabular}

A carbon nanotube can be different not only in its diameter or length but also by its chirality vector $(n, m)[75]$ that determine how the graphene sheet is rolled up to generate CNT. Usually, those CNTs with $n=m$ are classified as "armchair," while those with $\mathrm{m}=$ 0 are classified "zig-zag." Practically, most common CNTs existing in nature are neither "armchair" nor "zig-zag", but chiral; therefore it is difficult to model chiral type CNT in one simulation case. Since the focus of this work is the wettability of CNT with or without quadrupole potential, an "armchair" type CNT is selected to compose the CNT plate. The chiral vector of these CNT is $(6,6)$ with radius and length of $8.22 \AA$ and 24.74 $\AA$, respectively, as shown in Figure 5-1 with VMD software[82]. Table 5-2 gives the values of the parameters for water potential. 


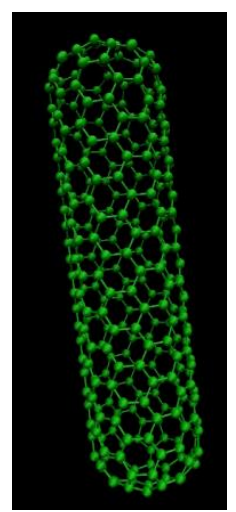

Figure 5-1 “Armchair” CNT

Table 5-2 potential function parameters of carbon related interaction

\begin{tabular}{ccc}
\hline Parameters & Values & Units \\
\hline$K_{C r}$ & 4.92 & $\mathrm{eV}^{-2}$ \\
$r_{C}$ & 1.418 & $\AA$ \\
$K_{C \theta}$ & 5.78 & $\mathrm{eV} \mathrm{rad}^{-2}$ \\
$\theta_{C}$ & $120.0^{\circ}$ & \\
$\varepsilon_{C C}$ & 0.0189 & $\mathrm{eV}$ \\
$\sigma_{C C}$ & 3.851 & $\AA$ \\
\hline
\end{tabular}

Morse potential is used to describe $\mathrm{C}-\mathrm{C}$ bond stretching and cosine-squared style is selected to represent potential energy storing in the $\mathrm{C}-\mathrm{C}-\mathrm{C}$ angle. Therefore, the total potential energy of a single CNT can be expressed as,

$$
U\left(r_{i j}, \theta_{i j k}\right)=K_{C r}\left(\xi_{i j}-1\right)^{2}+\frac{1}{2} K_{C \theta}\left(\cos \theta_{i j k}-\cos \theta_{C}\right)^{2}
$$

where, $\xi_{i j}=e^{-\gamma\left(r_{i j}-r_{C}\right)}$ and the interaction between carbon atoms are described as Lenard Jones potential with corresponding values of $\sigma_{\mathrm{CC}}$ and $\varepsilon_{\mathrm{CC}}[83]$.

The interaction between water molecule and CNTs is considered as combined effect contributed by pair Lennard-Jones potential and quadrupole potential among charged sites, while the quadrupole potential[84] is in the form of [83]: 


$$
U\left(r_{\alpha}, r_{\beta}\right)=\frac{1}{3} \frac{q}{4 \pi \varepsilon_{0}} \sum_{\alpha, \beta} \Theta_{\alpha, \beta} \frac{3 r_{\alpha} r_{\beta}-r^{2} \delta_{\alpha \beta}}{r^{5}}
$$

where $\alpha, \beta$ run over all Cartesian coordinates $x, y, z$ and $r$ is the distance between the charge site and the quadrupole carbon site. And $\delta_{\alpha \beta}$ is the delta function, and $\Theta_{\alpha, \beta}$ is the quadrupole moment tensor. The values applied to the potentials are given in Table 5-3.

Table 5-3 potential function parameters of water-carbon interaction

\begin{tabular}{ccc}
\hline Parameters & Values & Units \\
\hline$\varepsilon_{\mathrm{CO}}$ & 0.0091 & $\mathrm{eV}$ \\
$\sigma_{\mathrm{OO}}$ & 3.5196 & $\AA$ \\
$\Theta_{\alpha, \beta}$ & $3.03 \times 10^{-40}$ & $\mathrm{C} \mathrm{m}^{2}$ \\
\hline
\end{tabular}

All simulation is carried out with LAMMPS[36] which has quadrupole potential implemented under the latest version. The MD simulation work consists of two parts. One focus will be investigating how the separation distance affects the wettability of twin carbon nanotubes immersed in liquid water. Two carbon nanotubes are placed in liquid water box with a fixed distance varying from $8.5 \AA$ to $15 \AA$. The water box, with dimension of $63 \AA \times 63 \AA \times 75 \AA$, contains more than 9000 water molecules which may slightly vary e with different separation distance between two carbon nanotubes. The initial lattice of water is set to $3.10 \AA$ with cubic crystal structure such that the density of water is $1000 \mathrm{~kg} / \mathrm{m}^{3}$ at room temperature $(298 \mathrm{~K})$; periodic boundary conditions are applied to all three directions. All water molecules are integrated with Verlet algorithm within canonical ensemble. The simulation is performed with a cutoff distance of $20 \AA$ for pair potential and $25 \AA$ for electrostatic related potential, while the timestep is set to 1 fs. It is worth to notice that water molecules are only affected by pair potential and quadrupole potential between carbon atom and oxygen atom since only water molecules 
will be integrated in the simulation box in order to have a fixed separation distance. As the second part of this work, the contact angle of the droplet with same size sitting on a carbon nanotube plate will be measured after its equilibrium state is achieved. Similar to part one, the cases with/without quadrupole potential will be studied to investigate the significance of the quadrupole effect on wetting phenomena. A cubic water box with dimension of $20 \AA \times 20 \AA \times 20 \AA$ is equilibrated to sphere-like droplet by 200 ps before it is placed on carbon nanotube plate. The lattice of initial cubic droplet is set to be $4.91 \AA$ with face-centered cubic corresponding to liquid water density of $1000 \mathrm{~kg} / \mathrm{m}^{3}$ at room temperature.

\subsection{Results and Discussions}

The relationship between separation distance and wettability of carbon nanotube is studied first. The twin CNTs are immersed in water at room temperature with a fixed distance ranging from $8.5 \AA$ to $15 \AA$. Figure 5-2 gives snapshots of water molecules distribution within $10 \%$ of the simulation box along with CNT's axis direction. It should be pointed out that "NQ" means quadrupole potential disabled and "WQ" means "with quadrupole potential enabled." 


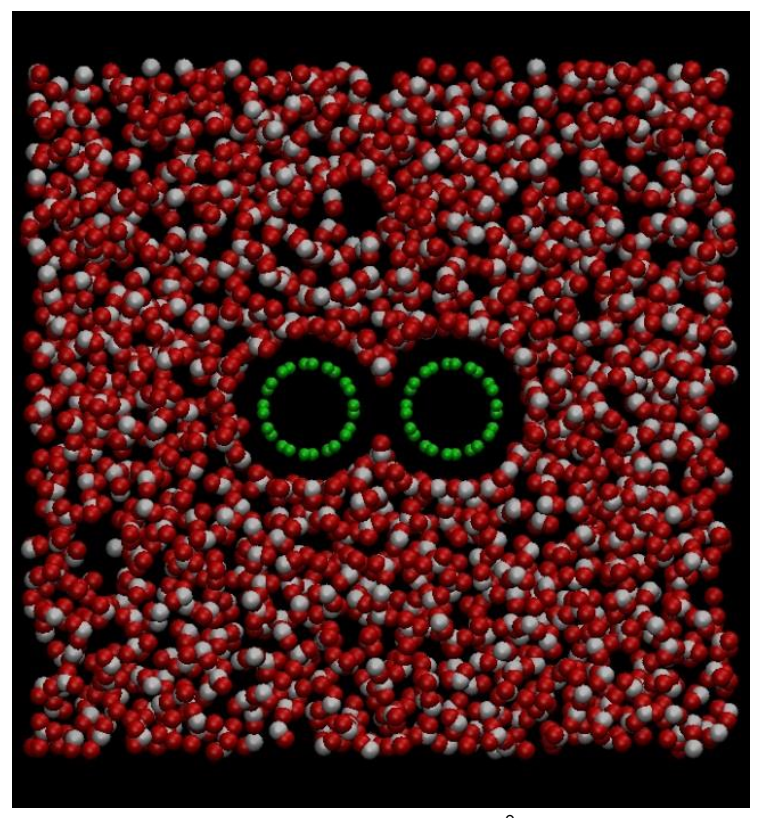

(a ) NQ, $d=13 \AA$

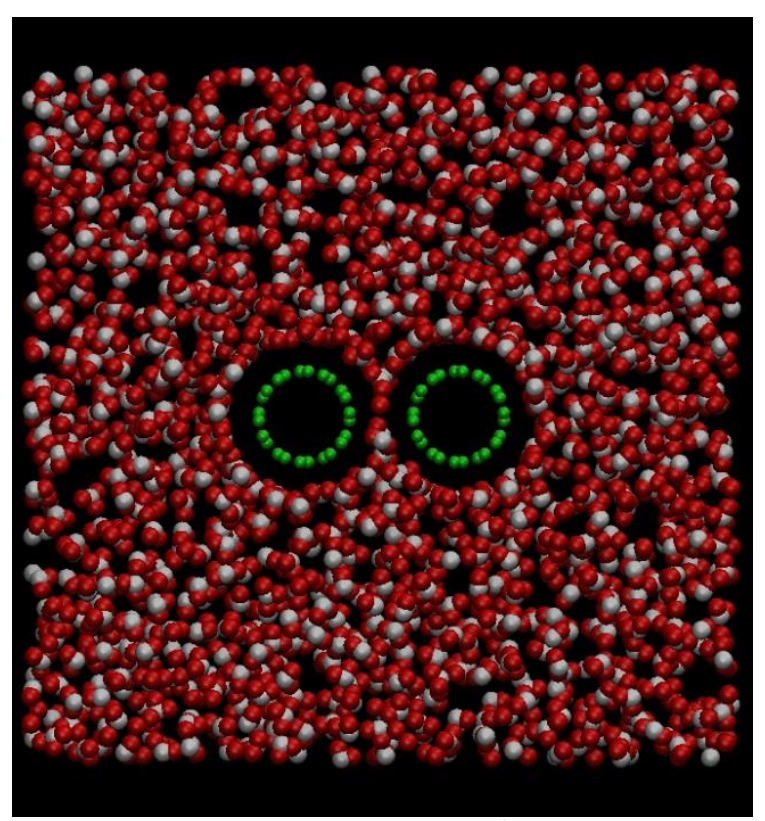

(c) NQ, $d=14 \AA$

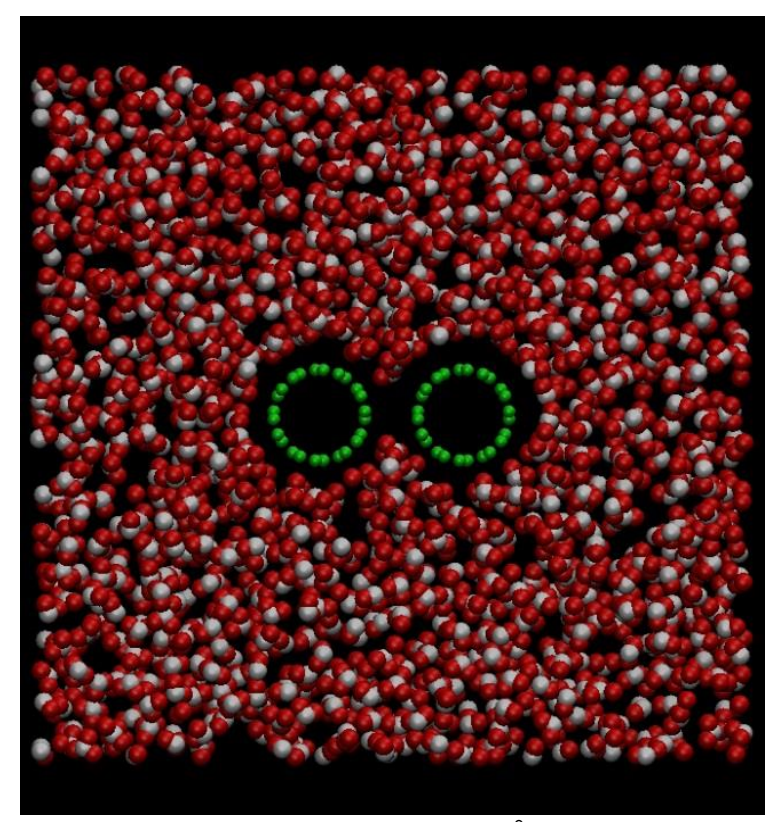

(b) WQ, $d=13 \AA$

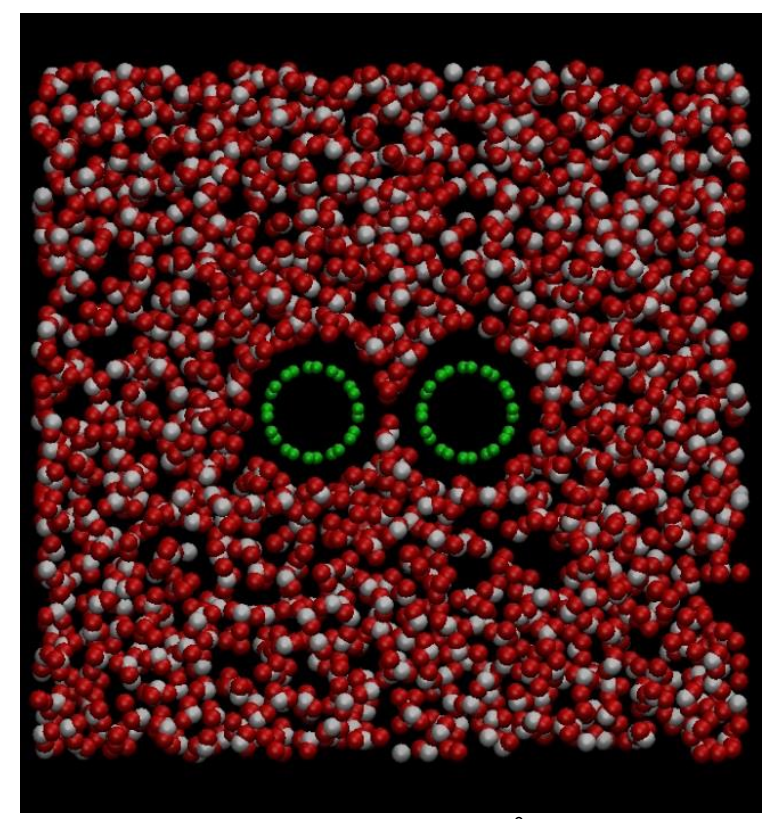

(d) WQ, $d=14 \AA$ 


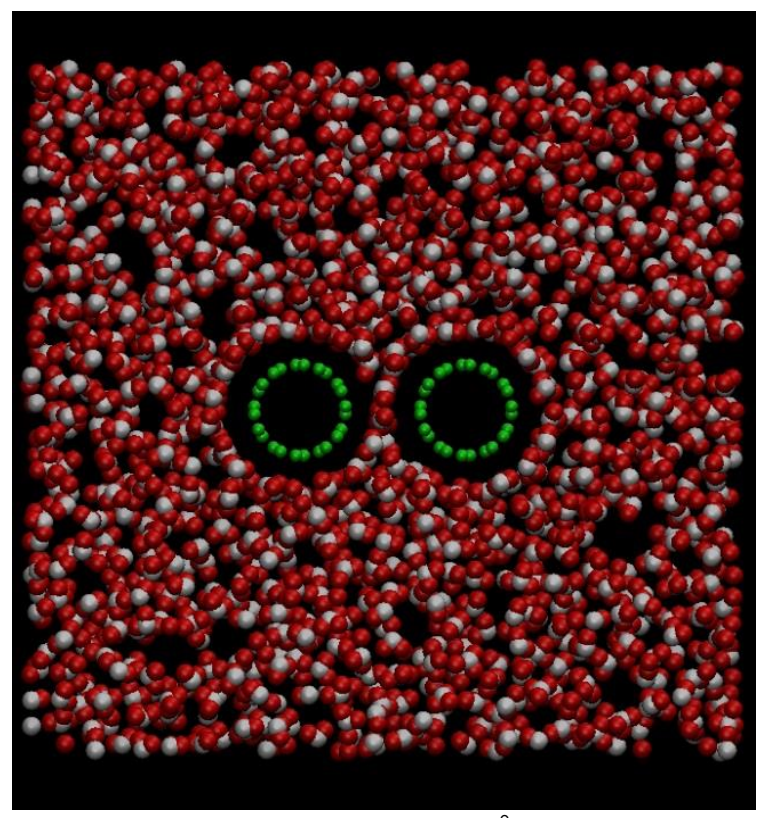

(e) NQ, $d=15 \AA$

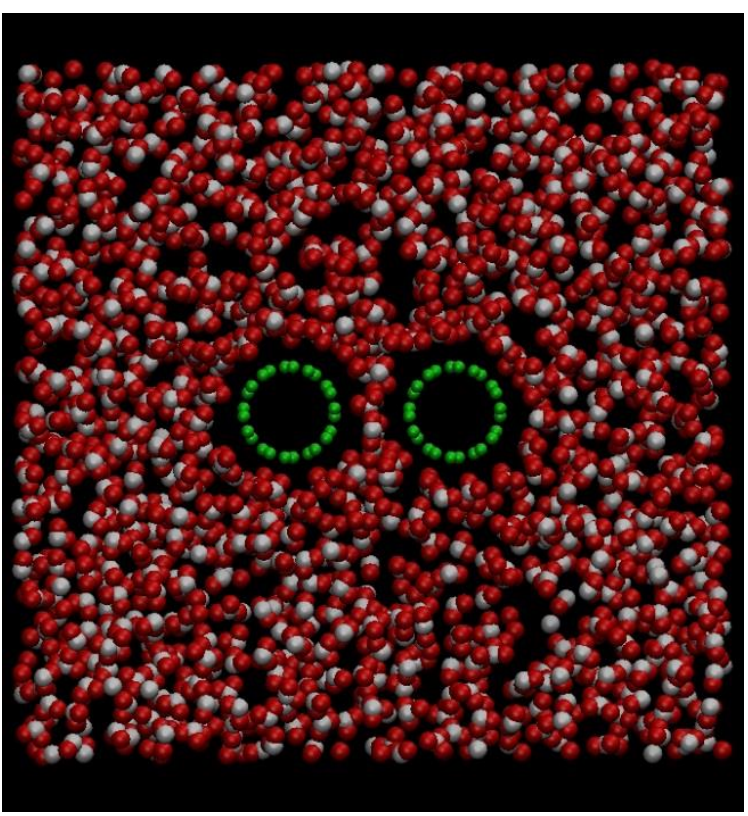

(f)WQ, $d=15 \AA$

Figure 5-2 Comparison of wettability of CNT with different separation distance

It can be seen that the critical value of wetting CNT aligned plate for both cases has values between $13 \AA$ and $14 \AA$. These results also demonstrated that quadrupole potential has a negligible effect on wetting CNTs which is in agreement with ref[82]. It is also interesting to notice that a stable configuration between water molecules and CNT is formed when the system is fully equilibrated. In other words, a certain thick vacuum region exists around each carbon nanotube. With the purpose of investigating the thickness of this vacuum region, the time- averaged minimum distance between center of mass of water molecule and central line of carbon nanotube tube is measured. The value for the cases without counting electrostatic quadrupole moment is around $3.15 \AA$, while the value is $3.02 \AA$ for those with quadrupole term enabled. It is found that the level of this plateau coincides with the thickness of 1.5 layer of water, which is also confirmed by Wallqvist and Berne[85].Therefore, it can be concluded that the electrostatic quadrupole moment only has a minor impact on wetting. Table 5-4 shows the obtained minimum 
distance for each case. In general, the cases with the quadrupole term appended have lower values, which could be caused by relatively larger attractive force to hydrogen than to repulsive force to the oxygen atom. Though electrostatic quadrupole momentum will lead to lower distance, the difference is only $4.3 \%$. Furthermore, it can be confirmed that quadrupole potential will cause minor difference in wetting carbon nanotubes.

Table 5-4 Minimum distance between water molecule and single CNT at equilibrium

\begin{tabular}{ccc}
\hline & $\mathrm{NQ}$ & $\mathrm{WQ}$ \\
\hline$d=8.5 \AA$ & $3.188 \pm 0.022 \AA$ & $3.028 \pm 0.023 \AA$ \\
$d=9.0 \AA$ & $3.172 \pm 0.022 \AA$ & $3.022 \pm 0.025 \AA$ \\
$d=10 \AA$ & $3.156 \pm 0.024 \AA$ & $3.021 \pm 0.029 \AA$ \\
$d=12 \AA$ & $3.195 \pm 0.020 \AA$ & $3.000 \pm 0.032 \AA$ \\
$d=13 \AA$ & $3.171 \pm 0.023 \AA$ & $3.027 \pm 0.031 \AA$ \\
$d=14 \AA$ & $3.134 \pm 0.031 \AA$ & $3.014 \pm 0.030 \AA$ \\
$d=15 \AA$ & $3.163 \pm 0.028 \AA$ & $3.012 \pm 0.025 \AA$ \\
\hline
\end{tabular}

As the second part of the simulation work, the contact angle of water droplet on various CNT composed plates are measured. Figure 5-3 shows the initial configuration of simulation box, where an equilibrated sphere-like water droplets is placed on the top of the plate. In order to investigate the quadrupole effect on droplet contact angle, a pair of cases with or without appending quadrupole term is studied. The only difference between all carbon nanotube plates is the separation distance which also varies from $8.5 \AA$ to 15 $\AA$.

Usually, when a liquid droplet with its vapor phase is placed on a solid substrate, as 
indicated by Young's equation, the three phases-liquid, solid, and vapor-reach a mechanical equilibrium when the three surface tension at interface between each phase balance at the contact line. However, it has been suggested that an addition term (line tension) should be included as a correction in order to properly describe the relationship between contact angle and surface tension[86]. In this work, the contact angle is measured by a popular used geometric technique which can be expressed as[87],

$$
\rho(r, z)=\frac{1}{2}\left(\rho_{l}+\rho_{v}\right)-\frac{1}{2}\left(\rho_{l}-\rho_{v}\right) \tanh \left[\frac{2\left(r-r_{e}\right)}{d}\right]
$$

where $\rho_{l}=1.0 \mathrm{~g} / \mathrm{cm}^{3}[30]$ and $\rho_{v}=0.59 \times 10^{-3} \mathrm{~g} / \mathrm{cm}^{3}$ [30] are the density of bulk liquid and vapor respectively, $r_{e}$ is the location of equimolar Gibbs dividing surface, and $d$ is the thickness of the phase transition. The detailed procedure is described as following. The time-averaged spatial density profile of the water droplet is obtained first by cylindrically binning the atoms around a reference axis perpendicular to the surface through the center of mass (COM) of the droplet. Variable $r$ in eq. (5.4) is the horizontal distance from the reference axis across the COM, and $z$ the height coordinate along the surface normal direction. The bins in the coordinate system used in the density distribution calculations have a height of $\Delta z=0.5 \mathrm{~nm}$ and a base area of $\Delta A=1 \sim 4 \mathrm{~nm}^{2}$, hence the radial bin boundaries being located at $r_{i}=\sqrt{i \Delta A / \pi}$ where $i=1,2,3 \ldots$ is an index for the radial bins. After the cylindrical density distribution is obtained, the phase transition part can be determined by fitting eq. (5.4) for each slab at z. Finally the fourth order polynomial fitting is carried out to obtain tangential value at $\mathrm{z}=0$ where is the position of the top surface of CNT plate, so that the contact angles can be calculated for each case. It should be mentioned that the $\mathrm{COM}$ of the liquid droplet is shifted to the origin point $\mathrm{O}$ such that 
a minimum error can be guaranteed when determining the reference axis that goes across the COM of the droplet. The fitting process was done by GNU scientific library[88]. The sample in total is 100 with time interval of 0.5 ps. Each case starts with the exactly same equilibrated droplet.

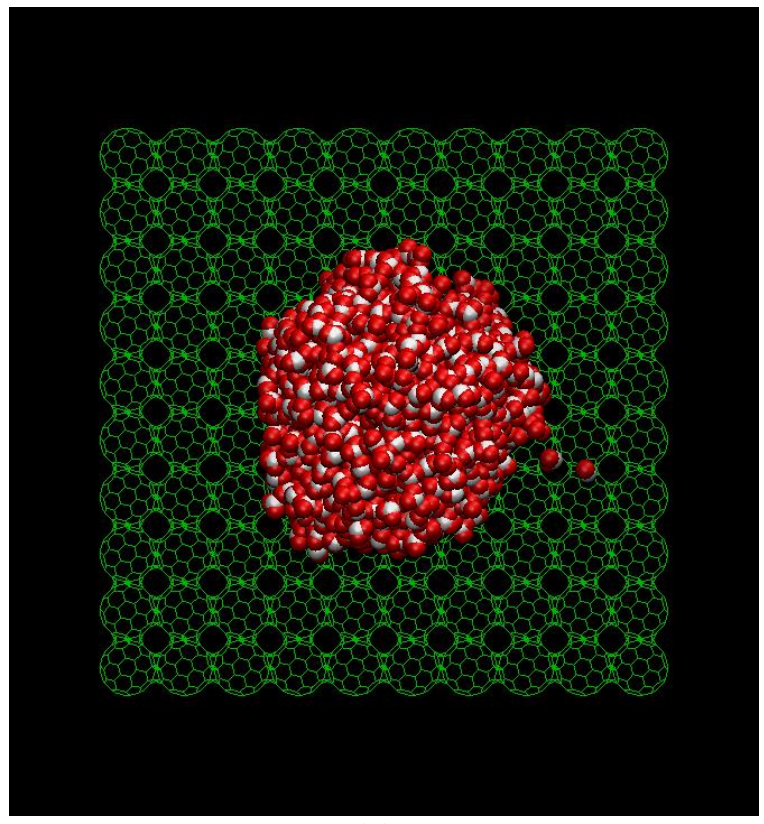

(a)

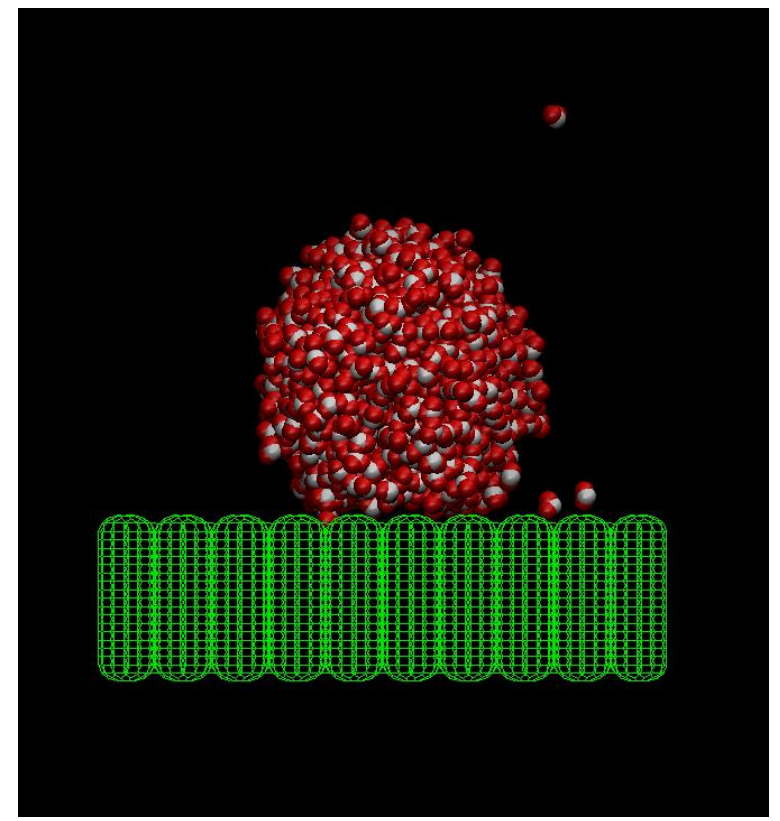

(b)

Figure 5-3 Initial configuration of simulation box $(d=8.5 \AA)$

It is found that the cases without quadrupole potential enabled cannot give a reasonable contact angle because the droplet collapses on the plate no matter how the separation distance varies. However, the droplets that have quadrupole potential effect accounted for can sit on the plate stably and hold their sphere-like shape well. Figure 5-4 gives a comparison of the pair cases with separation distance of $12 \AA$ to demonstrate. It can be seen that all water molecules collapse and piled up on the top of the CNT plate in Figure 5-4(a), while Figure 5-4(b) shows a water droplet with a few vapor molecules around stably sit on the plate at the end of the simulation. It is also shown in Figure 5-4(a) that lots of water molecule fell into the gaps between carbon nanotubes, many 
more than in Figure 5-4(b). In order to show the water molecule distribution among carbon nanotubes, Figure 5-5(a) and (b) corresponding to Figure 5-4(a) and (b) are given. It can be seen that the gap is not wetted, as confirmed by the previous results. Another observation is that many more water molecules stuck in the plate due to less repulsive force on oxygen atoms which could be caused by the special structure of capped-end of “armchair" carbon nanotubes.

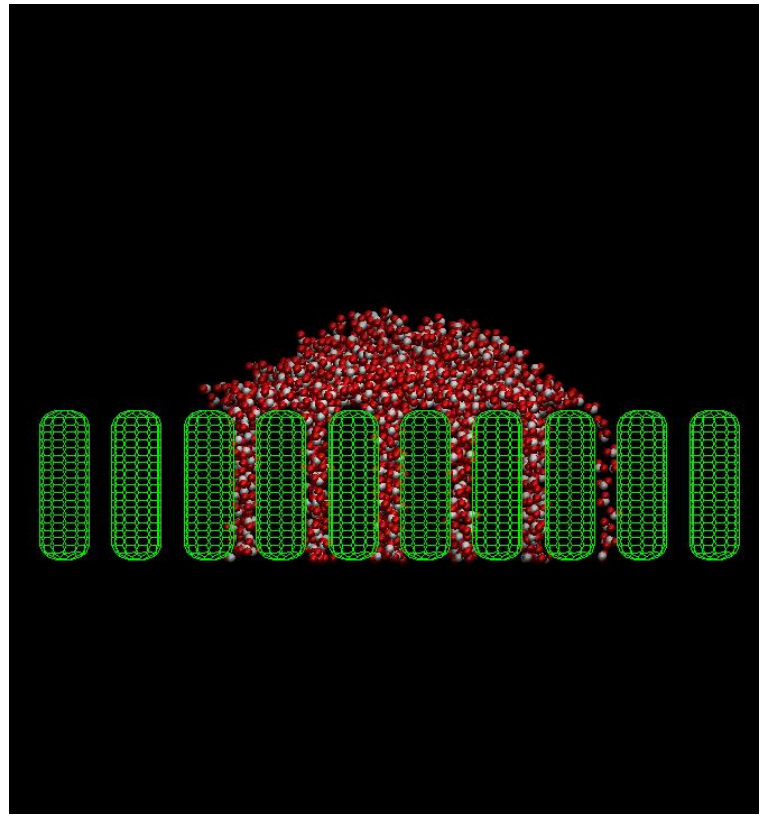

(a)

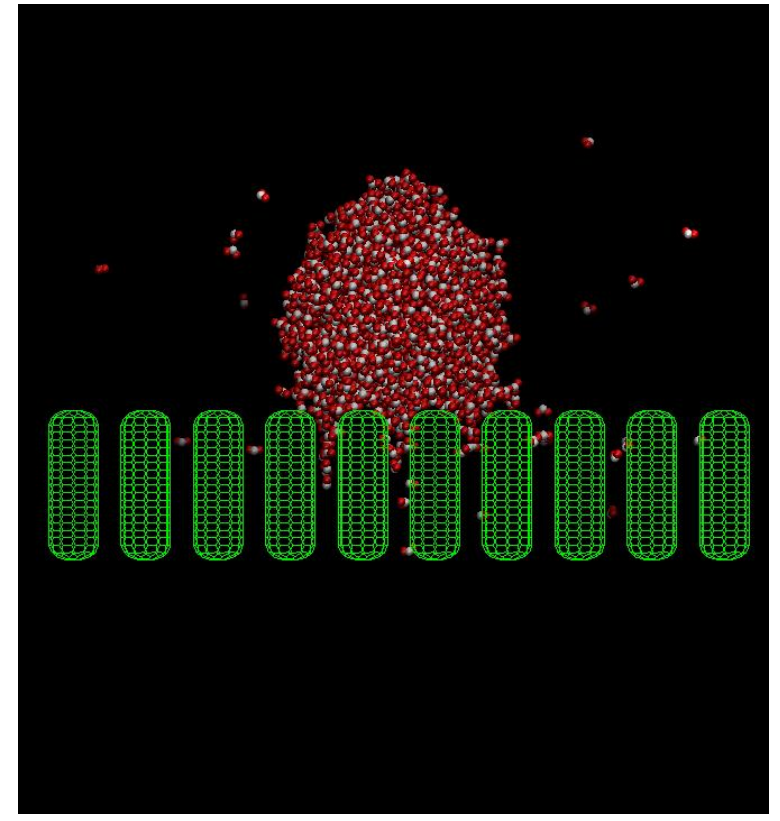

(b)

Figure 5-4 A comparison between two cases with/without quadrupole term ( $d=13 \AA)$

Table 5-5 gives those contact angles measured for each of the cases. ("N/A" indicates that a sphere-like droplet is not formed, thus, contact angle is not measurable). It is interesting to find that the contact angle is smaller than that reported in the literature. One obvious reason comes from the very different environment when measuring the contact angle. In real experiments, it is very hard to keep the purity of a CNT plate, not only in aspect of material but also in structure. However, the MD simulation does not account for factors associated with purity. Another main reason comes from the size 
effect. Currently, nearly all of the contact angles obtained in micro-scale are at the level of millimeter. However, the droplet size in this simulation is in the nano-scale. Though no direct experimental result can be used to compare with that from MD simulation, it is still meaningful to investigate the mechanism of hydrophobic phenomena of carbon nanotube at molecular level, because it is possible to count the hydrophobicity weight contributed through molecular interactions without introducing other uncontrollable affects.

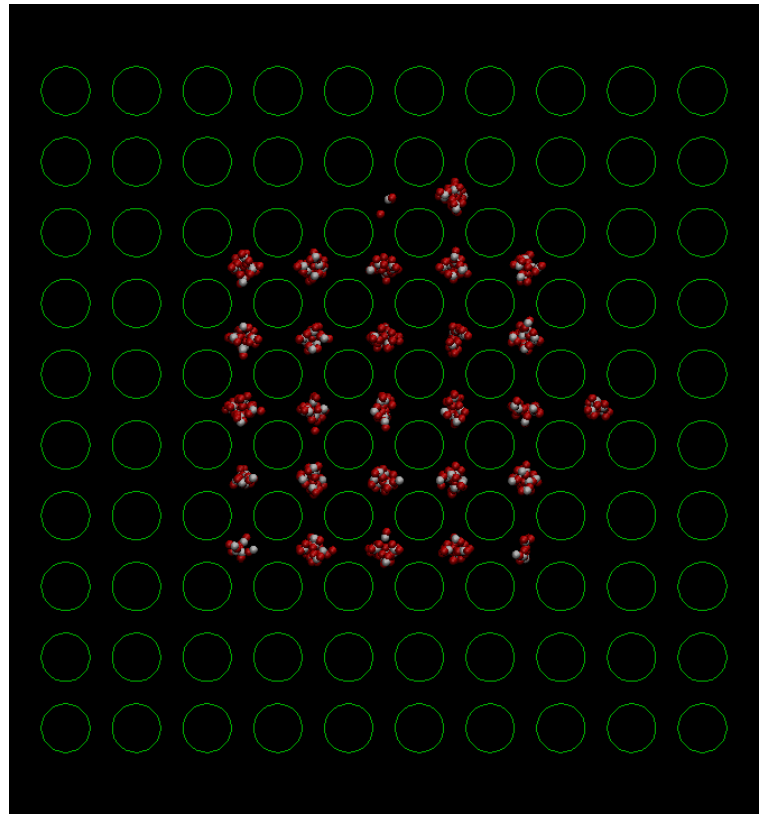

(a)

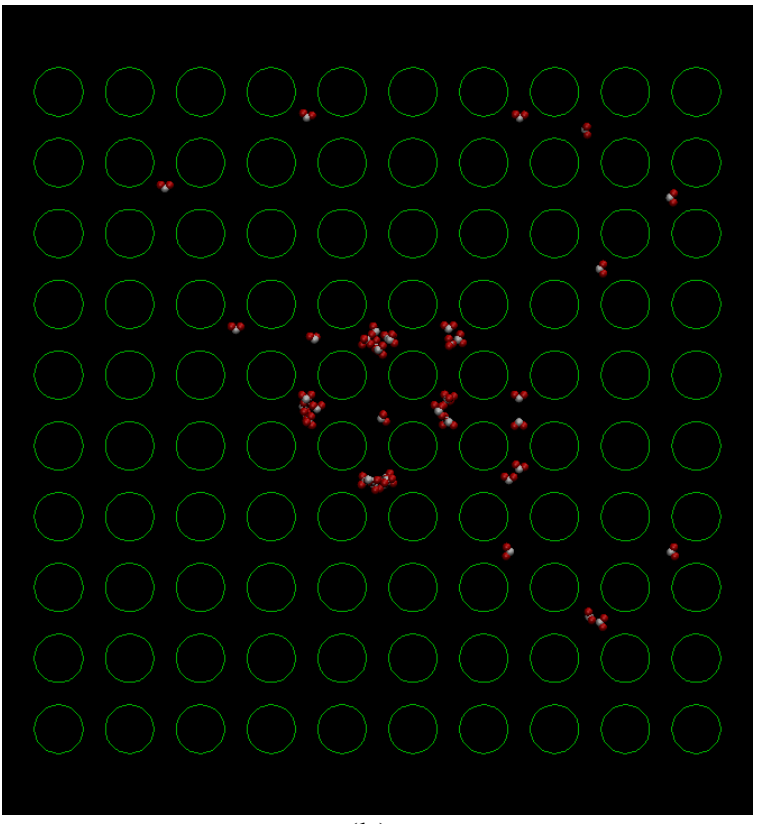

(b)

Figure 5-5 water molecule distribution with carbon nanotube plates $(d=13 \AA)$

Through Table 5-5, it is found that when the separation distance is $13 \AA$, it reached the maximum contact angle of $119.777 \pm 0.026^{\circ}$ which could make the plate as more hydrophobic. A distance of $14 \AA$ also produces a large contact angle of $106.519 \pm 0.018^{\circ}$, while for the other cases; carbon nanotube plate even cannot be hydrophobic, like the one with separation distance $8.5 \AA$. Thus, it can be concluded that separation distance will significantly affect the hydrophobicity of carbon nanotube plate based on current simulation results. 
Table 5-5 contact angles of droplet sitting on various CNT plate with/without quadrupole potential

\begin{tabular}{lcc}
\hline & $\mathrm{NQ}$ & $\mathrm{WQ}$ \\
\hline$d=8.5 \AA$ & $\mathrm{N} / \mathrm{A}$ & $38.288 \pm 0.044^{\circ}$ \\
$d=9.0 \AA$ & $\mathrm{N} / \mathrm{A}$ & $56.403 \pm 0.038^{\circ}$ \\
$d=12 \AA$ & $\mathrm{N} / \mathrm{A}$ & $95.959 \pm 0.026^{\circ}$ \\
$d=13 \AA$ & $\mathrm{N} / \mathrm{A}$ & $99.209 \pm 0.019^{\circ}$ \\
$d=14 \AA$ & $\mathrm{N} / \mathrm{A}$ & $119.777 \pm 0.026^{\circ}$ \\
$d=15 \AA$ & $\mathrm{N} / \mathrm{A}$ & $106.519 \pm 0.018^{\circ}$ \\
& $\mathrm{N} / \mathrm{A}$ & $59.008 \pm 0.029^{\circ}$ \\
\hline
\end{tabular}

\subsection{Conclusions}

Interactions between "armchair" CNT plate and nano-sized water droplets are studied by molecular dynamics. It is found that the quadrupole term which act on the charged sites of the water has a minor influence on wetting process to twin carbon nanotubes. The critical wetting distance between carbon nanotubes is a value between $13 \AA$ and $14 \AA$ no matter if quadrupole term is included or not to the water molecule potential. Quantitatively, the minimum distance between carbon nanotube and water molecules at equilibrium is $3.02 \AA$ for quadrupole enabled cases, and $3.15 \AA$ for the quadrupole disabled cases. The quadrupole term will reduce such distance, but the difference is only $4.3 \%$. Thus, it is still safe to conclude that the quadrupole term has a negligible effect on the wetting process of carbon nanotubes.

Quite differently from the wetting process, the quadrupole term turns to be a very important factor when a liquid droplet is interacting with CNT plate. For those cases 
without quadrupole term appended, all the droplets collapsed while those cases with quadrupole terms enabled can form a sphere-like droplet and sits stably on the plate. A critical separation distance of a value around $13 \AA$ is found to obtain the maximum contact angle of nano-sized droplet. Contact angle of liquid water is very sensitive to separation distance, due to the fact that, it is found that the plate cannot be even called hydrophobic for some cases, like that with separation distance $8.5 \AA$.

\section{Acknowledgement}

Support for this work by the U.S. National Science Foundation under grant number CBET- 1066917 is gratefully acknowledged. 


\section{Chapter 6 Molecular Dynamics Simulation on Rapid Boiling of Water on a Hot Copper Plate}

\subsection{Introduction}

In a normal boiling process, the vapor bubble is generated heterogeneously near the heat wall. When the liquid is superheated to a degree much higher than the normal saturation temperature and approaching the thermodynamic critical temperature, homogeneous vapor bubble nucleation takes place at an extremely high rate, which lead to the near-surface region of the materials being ejected rapidly[89]; this process is referred to as rapid boiling. The rapid boiling is also referred to as vapor explosion, phase flashing, thermal detonation, and rapid phase transitions. Many special events occur during rapid boiling, such as pressure shock wave, bubble clusters formed by tiny bubbles, and high superheat. Many experimental works are reported [90-93], but these experimental works are limited in either length or time scales. Due to the limitation of classical macroscopic theory, many special phenomena in phase explosion cannot be well explained.

A molecular dynamics (MD) simulation, which has advantages of describing any physical process at atomic level, is widely applied to study micro- and nanoscale heat and mass transfer problems. Dou et al. presented a microscopic description on rapid boiling of water films adjacent to heated gold surface, and found that the vaporization phenomena highly depended on the initial thickness of the water film[94]. Gu and Urbassek performed MD simulation on rapid boiling of liquid-argon films irradiated by ultrafast laser[95]. Zou et al. simulated a homogenous nucleation of water and liquid 
nitrogen in the rapid boiling, and energy conversion and redistribution were studied[96]. Though the existing MD work have provided some molecular level understandings of the phase transition in the rapid boiling, few work revealed the thermal and dynamic mechanisms caused by an extremely high heat flux through metal plate, like copper. Moreover, few researchers paid attentions on the dynamic phenomenon associated with different phases after the rapid boiling, although mechanical factor (like thermal stress) was always considered as an important effect determining machining performance in most applications. A study of water film's mechanical behavior is important from both fundamental and practical viewpoints, due to the trends of miniaturization of devices (MEMS, NEMS, etc.) and a demand by the development in much other technology (water photo-electrolysis[97], boundary lubrication[98], etc.).

In this paper, both thermal and dynamic phenomena of an ultrathin water film will be investigated during and after rapid boiling.

\subsection{Physical Models and Methods}

The computational domain is divided into three regions, namely vapor, liquid and solid region. Both vapor and liquid regions are filled with water molecules, and the solid is a copper plate. In consideration of accuracy in description of water's dynamic and thermal properties, a four-site water model (TIP4P)[57] is adapted to model vapor and liquid water. The well-accepted water molecular potential function, which consists of the contributions from electrostatic, dispersion and repulsive forces, is used to describe intermolecular interaction of water molecules:

$$
U_{a b}=\sum_{i}^{a} \sum_{j}^{b} \frac{k_{C} q_{a_{i}} q_{b_{j}}}{r_{a_{i} b_{j}}}+\sum_{i}^{a} \sum_{j}^{b} 4 \varepsilon_{a_{a}, b_{j}}\left[\left(\frac{\sigma_{a_{b} b_{j}}}{r_{a, b_{j}}}\right)^{12}-\left(\frac{\sigma_{a_{i} b_{j}}}{r_{a_{i} b_{j}}}\right)^{6}\right]
$$


where, $a$ and $b$ denote two different molecules, subscript $i$ and $j$ represent atoms of hydrogen or oxygen in one individual TIP4P molecule, and $k_{\mathrm{c}}$ is the electrostatic constant. The long-range columbic contribution to the entire system is computed by PPPM (particle-particle particle-mesh) approach[32] with an accuracy of $1.0 \times 10^{-6}$. It should be pointed out that the pair potential only counts the interaction between oxygen atoms within the cutoff distance of $12 \AA$; both bond and angle interactions within a single water molecule are considered as rigid, thus SHAKE[33] algorithm is applied to each water molecule to hold its geometry shape such that a longer time step of $1 \mathrm{fs}$ can be utilized. The water region is built with face-center cubic unit (FCC) with lattice constant of $37.0 \AA$ and $3.103 \AA$, respectively, subject to the densities of vapor and liquid at 1 atm[37]. For the copper plate, it is also modeled with face-centered cubic unit (FCC) with the lattice constant of $3.615 \AA$, which is consistent with the density of $8.9 \times 10^{3} \mathrm{~kg} / \mathrm{m}^{3}$. It should be mentioned that the interaction between copper atoms are not considered, instead, many artificial $\mathrm{Cu}-\mathrm{Cu}$ harmonic bonds are created for the plate to introduce $\mathrm{Cu}-\mathrm{Cu}$ spring-like interaction, which will be discussed later. The interaction between copper and oxygen or hydrogen atoms are considered using the Lenard Jones potential.

Table 6-1 water potential parameters

\begin{tabular}{ccc}
\hline Parameters & Values & Units \\
\hline$\varepsilon_{O O}$ & 0.006998 & $\mathrm{eV}$ \\
$\sigma_{O O}$ & 3.16438 & $\AA$ \\
$q_{\mathrm{H}}$ & 0.52 & $\mathrm{e}$ \\
$q_{\mathrm{O}}$ & -1.04 & $\mathrm{e}$ \\
$\varepsilon_{C u O}$ & 0.06387 & $\mathrm{eV}$ \\
$\sigma_{C u O}$ & 2.7172 & $\AA$ \\
$\varepsilon_{C u H}$ & 0.03396 & $\mathrm{eV}$ \\
$\sigma_{C u H}$ & 1.335 & $\AA$ \\
$E$ & $274 \sim 306$ & $\mathrm{GPa}$ \\
$d$ & 3.615 & $\AA$ \\
$v_{s}$ & 3901 & $\mathrm{~m} / \mathrm{s}$ \\
\hline
\end{tabular}


In order to introduce a more physically-sound thermostat to create heat flux through copper plate rather than artificially rescaling velocity of atoms, the copper plate is modeled using the approach described in[99]. As shown in Figure 6-1, five layers of atoms are created in FCC configuration. The top three layers (white) are "real" copper atoms.

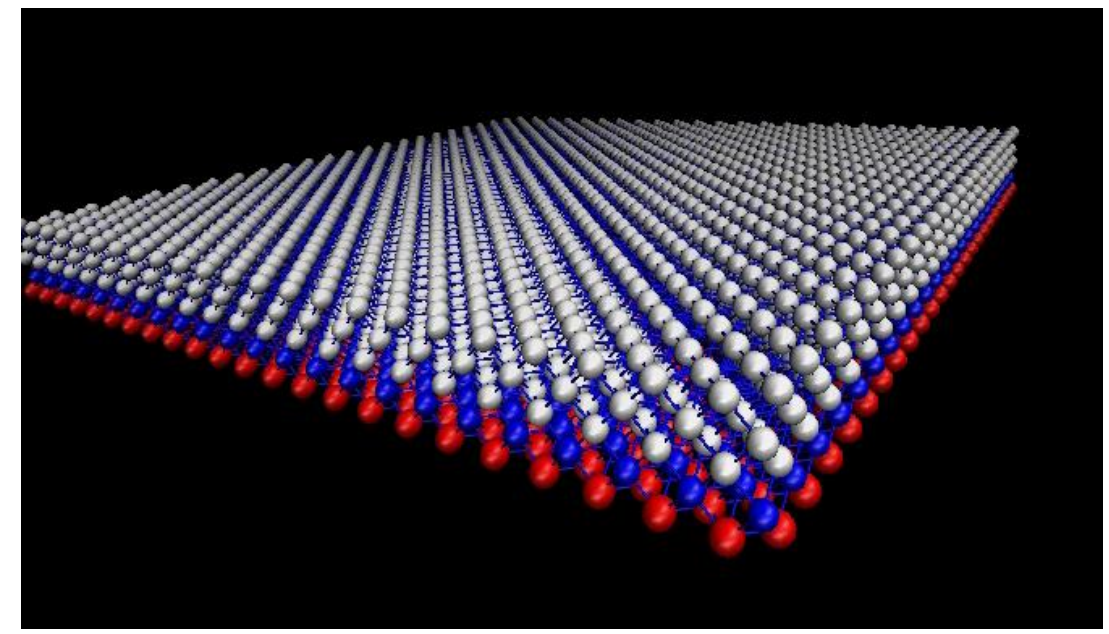

Figure 6-1 Structures of the copper plate, artificial harmonic bond are created by connecting neighbor atom within a distance of $2.56 \AA$

The fourth layer (blue) is considered to be phantom atoms exerted with a force combined with a damping force and random force that subject to Gaussian distribution. The fifth layer is fixed in order to prevent atoms from penetration. The standard deviation of the random force is,

$$
\sigma_{F}=\sqrt{\frac{2 \alpha k_{B} T}{\Delta t}}
$$

where $\alpha$ is dependent on desired temperature and integration time length, 


$$
\alpha=\frac{m \omega_{D} \pi}{6}
$$

where Debye frequency $\omega_{D}$ can be estimated by,

$$
\omega_{D}=\left(\frac{3}{4 \pi} \frac{N}{V}\right)^{\frac{1}{3}} v_{s}
$$

where $v_{s}$ is the speed of sound in the solid. Thus, the energy flux to the simulation system can be accurately calculated by integrating the exciting force and the damping force applied to those phantom atoms. It can also be seen from Figure 6-1 that an artificial harmonic bond is created by connecting neighbor copper atoms that are within its shortest distance of $2.56 \AA$. Since the interaction between atoms within the second layer of phantom atom is modeled with artificial harmonic bond, it is important to use a reasonable spring constant to obtain an accurate thermostat. The interatomic spring constant $k$ is tightly related to Young's Modules thus it could be estimated with formula below,

$$
k=E d
$$

where $E$ is Young's Modules of solid copper, and $d$ is corresponding lattice constant of solid metal. Table 6-1 gives all the parameters required in this simulation.

The simulation is carried out within the framework of the open Source MD code LAMMPS[36]. The entire simulation box has both length and width of $82.89 \AA$, and a height of $240 \AA$ A. Periodic boundary conditions are applied to all directions parallel to the surface of the plate, while a reflecting wall, which will bound the molecules back when they pass through the wall, is placed at the top to seal the simulation box. No energy transfer will occur through the interaction between molecules and this wall. By creating this wall, the water film can be successfully confined in the simulation box, and form a 
stable floating film during the later period of rapid boiling. At the same time, an empty space is included in the z-direction to remove dipole interactions between the slab and its images by extending the volume by two times. The water regions are filled with 12,504 molecules ( $\sim 6.2 \mathrm{~nm}$ in thickness) and there are 5,290 copper atoms in the hot plate. It is worth to mention that the size of simulation box and number of water molecules is chosen based on the water density at $298 \mathrm{~K}$ and 1 atmosphere pressure. Before the liquid water is heated by the hot plate, the system must be equilibrated in the following two steps: (1) the entire water zones are first equilibrated with certain thermostat (Berendsen thermostat)[35] until the temperature of water system was equilibrated to a stable value of $298 \mathrm{~K}$, and (2) the copper plate is then heated to $1000 \mathrm{~K}$ with "phantom atom" thermostat as stated above while the water molecules are isolated from the integration. After those preparations steps are done, the liquid water at $298 \mathrm{~K}$ is suddenly placed on the hot plate. The entire system is still integrated with NVE ensemble during the simulation and copper plate is still controlled to desired temperature of $1000 \mathrm{~K}$ with "phantom atom" thermostat. The value of $1000 \mathrm{~K}$ is chosen to guarantee that the temperature above the threshold as above in bubble formation[100] while it is still below the melting point of copper.

\subsection{Results and Discussions}

The monitored temperatures of the copper plate and entire water region are recorded and shown in Figure 6-2. 


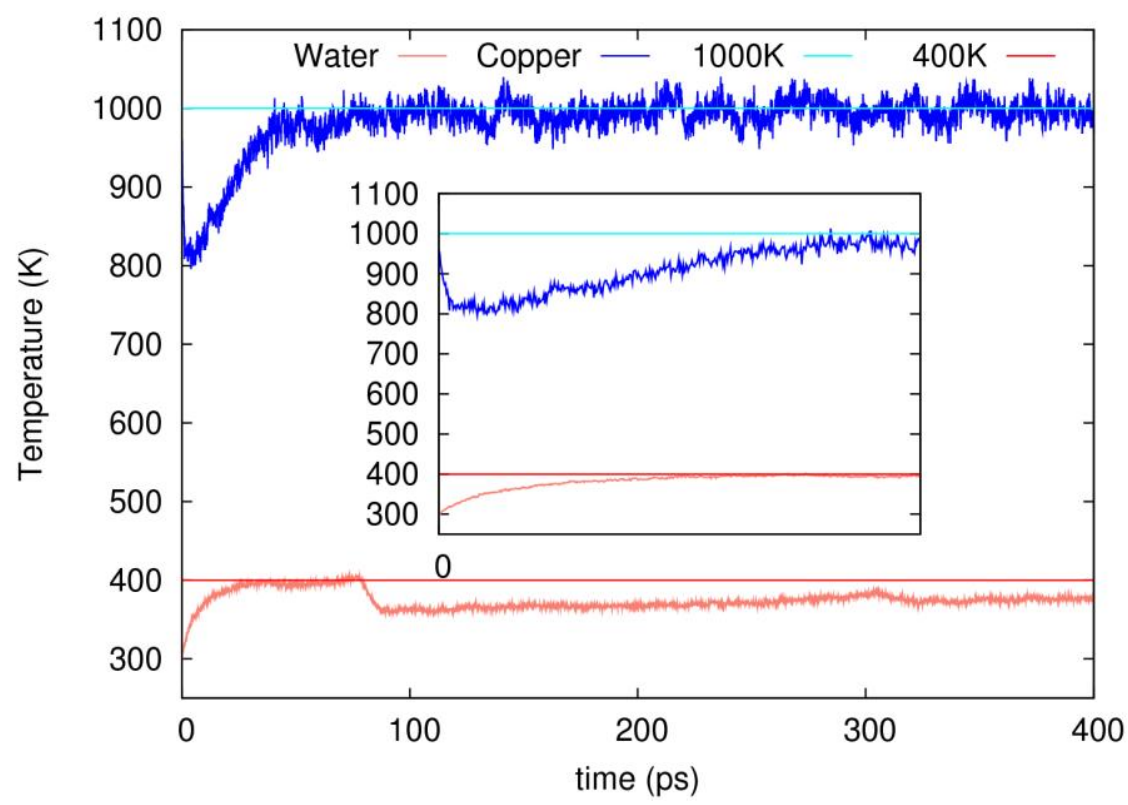

Figure 6-2Temperature variation of water and hot copper plate

It can be seen that there is an obvious temperature drop from $1000 \mathrm{~K}$ to a minimum value of $800 \mathrm{~K}$ when the "cold" water film touches the hot plate due to heat conduction mechanism of solid copper. It takes around $50 \mathrm{ps}$ before the temperature of the hot copper turning back to $1000 \mathrm{~K}$ the continuingly added heat flux. The temperature of water region keeps increasing from $298 \mathrm{~K}$ to $400 \mathrm{~K}$ during the initial $80 \mathrm{ps}$. 

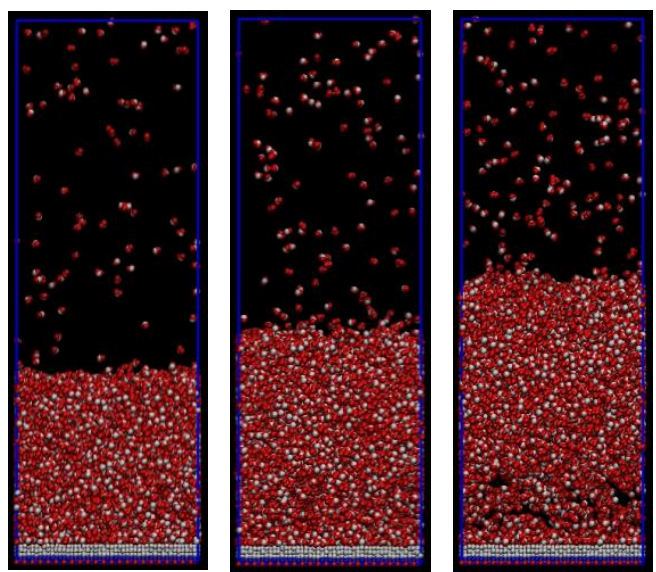

(a) $10 \mathrm{ps}(\uparrow)$

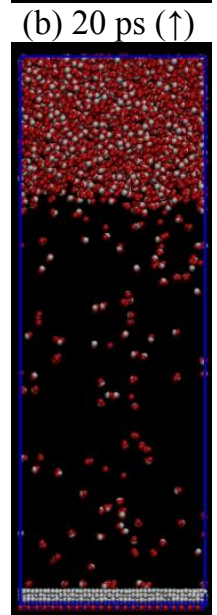

(c) $30 \mathrm{ps}(\uparrow)$
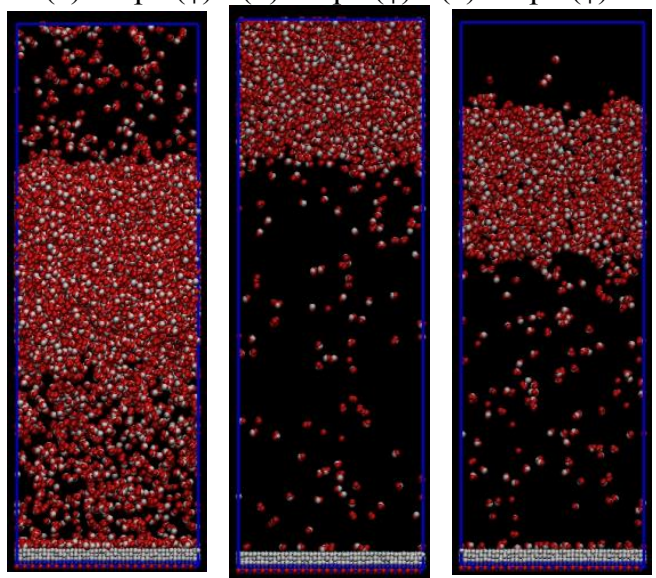

(d) $50 \mathrm{ps}(\uparrow)$

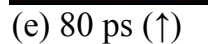

(f) 120 ps $(\downarrow)$
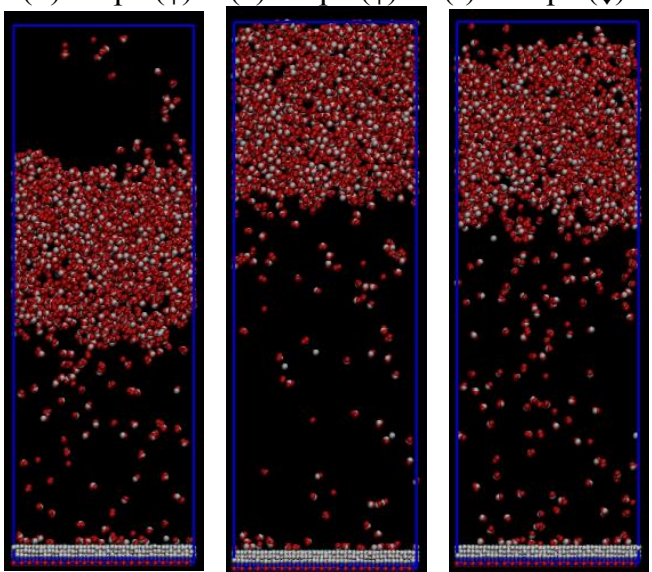

(g) $200 \mathrm{ps}(\downarrow)$

(h) $300 \mathrm{ps}(\uparrow)$

(i) $400 \mathrm{ps}(\downarrow)$

Figure 6-3 Snapshots of water molecule distribution through time $10 \mathrm{ps}$ to $400 \mathrm{ps}$ 
Figure 6-3 gives several snapshots of molecules spatial distribution at representative times (the arrow in the parenthesis denotes the moving direction of the bulk fluid). An obvious volume expansion of liquid water can be observed during the period from 10 to 20 ps by measuring the height of the liquid film. A low density vapor region appears at time of $30 \mathrm{ps,}$, and the entire water domain can be clearly divided into three regions: top vapor region, liquid region and the lower vapor region near the hot plate. Due to continuous heat flux absorbed by water molecules, the lower vapor region keeps expanding up to $80 \mathrm{ps}$ when the water film collide with the top wall. During this period, the entire wall surface is covered by vapor and the water film floats on the top of the vaporized water molecules layer in a manner reminiscent of the Leidenfrost phenomenon [101]. After the elastic collision with the wall, the film is forced to move backward, as shown in Figure 6-3(f). At approximately 200 ps, water film starts moving upward again due to larger pressure in the lower vapor region. The film hits against the top wall and rebound at $300 \mathrm{ps}$ and suspended at $400 \mathrm{ps}$ again. In corresponding to piston-like motion of liquid film, the COM's trajectory (z-component) of water region is recorded and shown in Figure 6-4. 


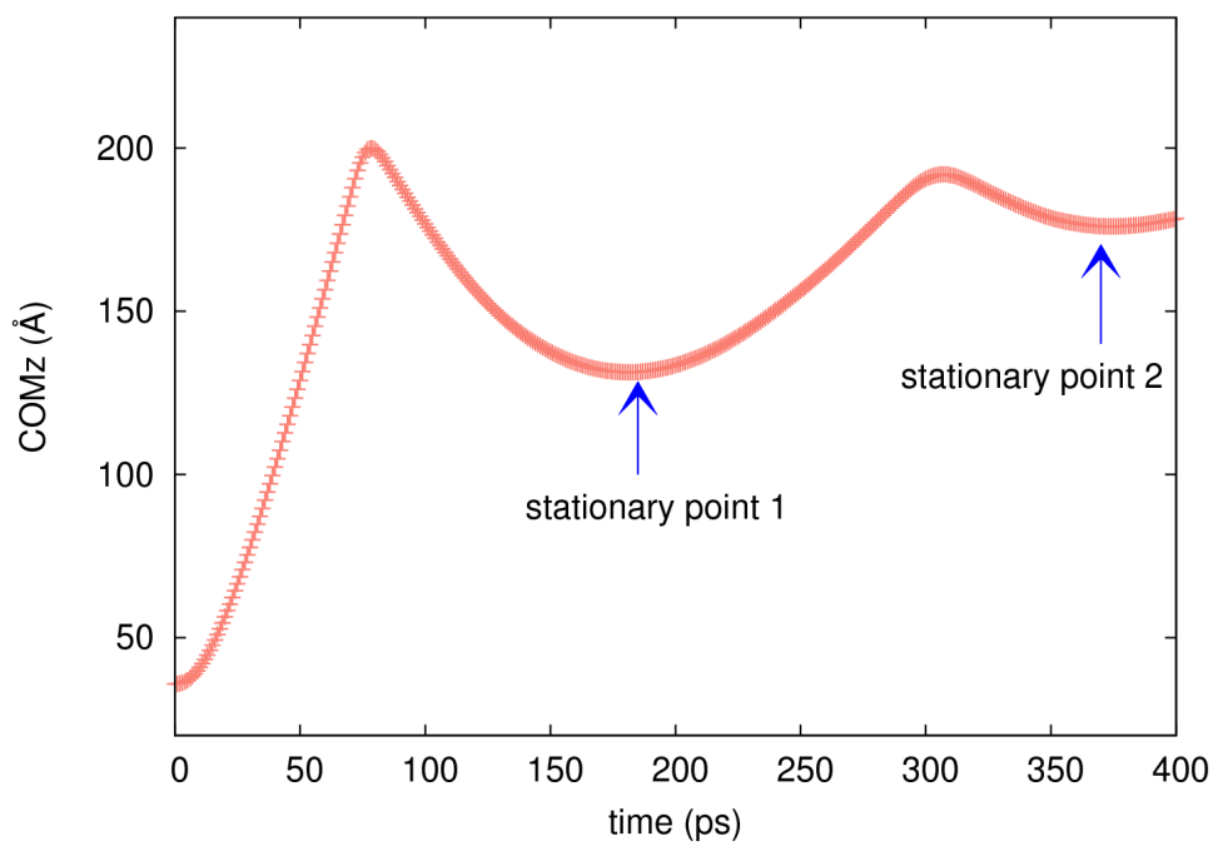

Figure 6-4 z-component of $\mathrm{COM}$ associated to water molecules within the simulation box

The period from 0 to $80 \mathrm{ps}$ is rapid boiling transition, and the rest is the bulk liquid's piston-like motion moment. It can be seen that the stationary point is flatter and higher, which means that the bulk liquid water will be stationary and eventually suspended somewhere adjacent to the wall due to the fact that the system keeps receiving the constant flux through copper plate.

In order to have a closer look at the density variation, the spatial density profile is computed by averaging density in 63 bins which are built by uniformly chopping the simulation box in the vertical direction; the final profile of density are shown in Figure 6-5 and the bulk liquid water moving directions are denoted by the arrow. It can be seen that the maximum bulk liquid density keeps decreasing during the moving process until it impact with the wall (see Figure 6-5(a)) due to rapid boiling. The increasing of the maximum density at $80 \mathrm{ps}$ comes from the water molecules' compression due to their 
inertia when they hits against the wall. In Figure 6-5(b), the maximum density also decreases when the bulk liquid moving backward mainly due to liquid expansion. Comparison between Figure 6-5(c) and (d) indicates that the maximum density does not change anymore and the height of the bulk liquid water keeps almost a constant; this indicates that phase change at the liquid-vapor interface is balanced during this period. Interestingly, a non-vaporized water molecule layer always exists at the interface between solid copper and lower vapor region no matter how long the heating processing lasts. This special zone, which is denoted as "hot gas-like zone", is also observed during evaporation process[102]. The density of this zone decreases during the period of rapid boiling and gradually stabilized at $0.2 \mathrm{~g} / \mathrm{cm}^{3}$. Figure 6-6 shows the variation of the "hot gas-like" water molecule distribution on the surface of the copper plate. From 0 to $60 \mathrm{ps,}$ it can be seen more and more localized "dry" region appears on the wall surface. However, during the later period, a fixed number of hot gas-like water molecules attached on the copper plate even continuous heat flux flows through the plate to water regions. This phenomenon could be raised by disjoining pressure that arise from Van der Waals attractive interaction between two parallel layers[103], here, by lower surface of water vapor and top surface of copper plate. 


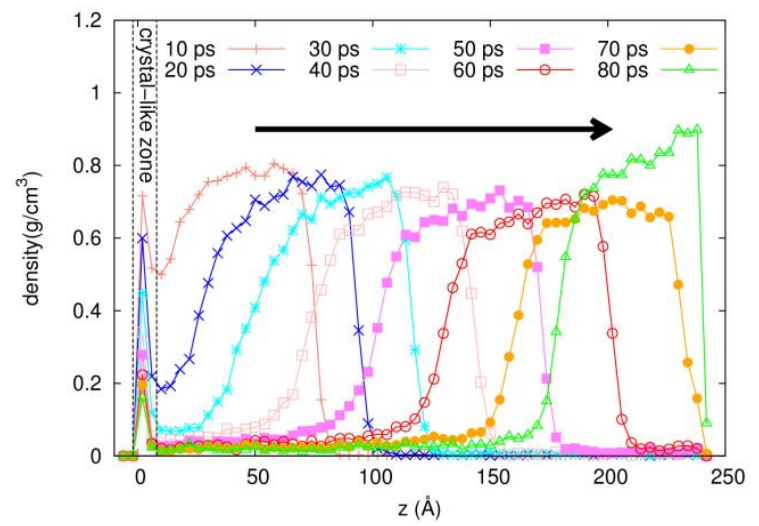

(a) $10-80 \mathrm{ps}$

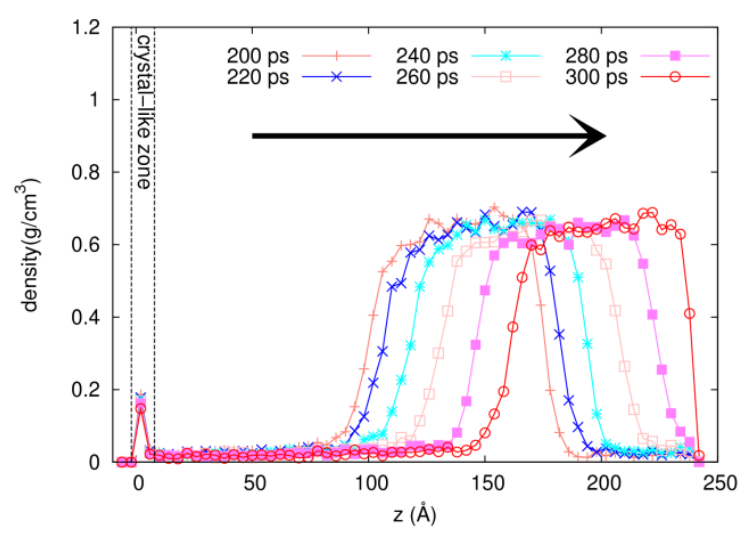

(c) $200-300 \mathrm{ps}$

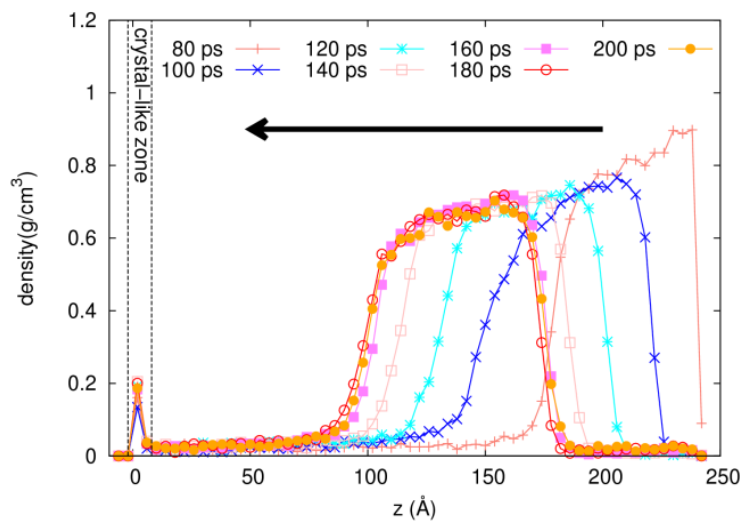

(b) $80-200 \mathrm{ps}$

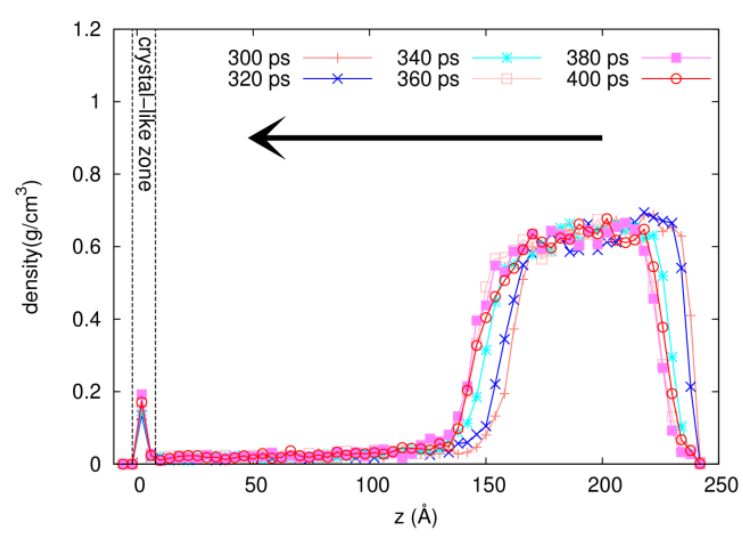

(d) $300-400 \mathrm{ps}$

Figure 6-5 One-dimensional spatial density distribution at various times 
The temporal density profile is also computed and rendered into a 2-D plot in Figure 6-7 where the vertical axis is the distance measured from the surface of the copper plate and the horizontal axis is the simulation time. The band that represents the bulk water film becomes narrower and narrower during the period of rapid boiling (0-80ps). It expands a little back to original thickness and keeps constant due to phase transition balance at the interface, which is consistent with the results in Figure 6-5.
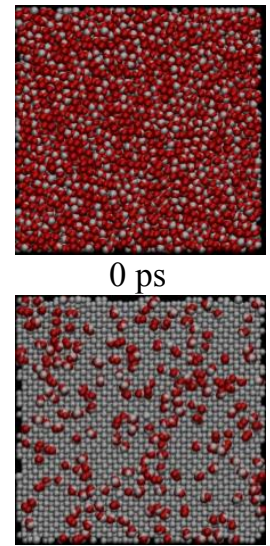

$60 \mathrm{ps}$

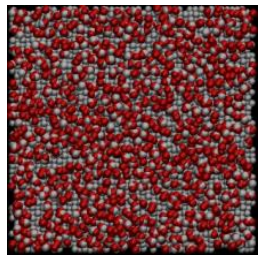

20 ps

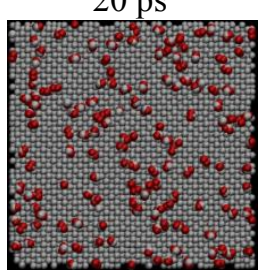

$80 \mathrm{ps}$

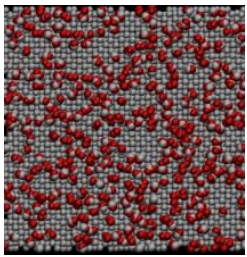

$40 \mathrm{ps}$

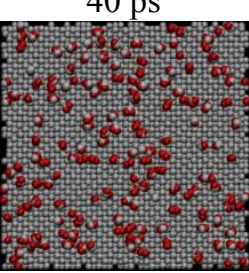

400 ps

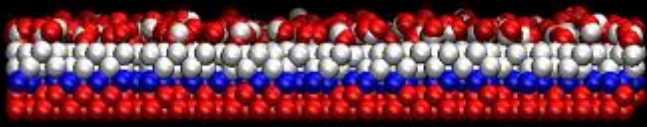

400ps side view

Figure 6-6 Top views of water molecules distribution on the surface of the copper

plate

Figure 6-8 shows the temperature variation in each region by dynamically detecting the liquid-vapor interfaces. During the period of rapid boiling, temperature in the lower vapor region is much higher than middle liquid film and top vapor regions. The maximum temperature of lower vapor region is $780 \mathrm{~K}$ which is only $220 \mathrm{~K}$ below the initial temperature of the plate $(1000 \mathrm{~K})$. In consideration of the temperature drop of the plate, the temperature difference between bottom vapor and copper plate is even smaller. 
For the lower vapor region, it can be seen that temperature suddenly fell at around $45 \mathrm{ps}$ when the vapor regions is fully expanded such that the amount of kinetic energy of molecules are converted to potential energy.

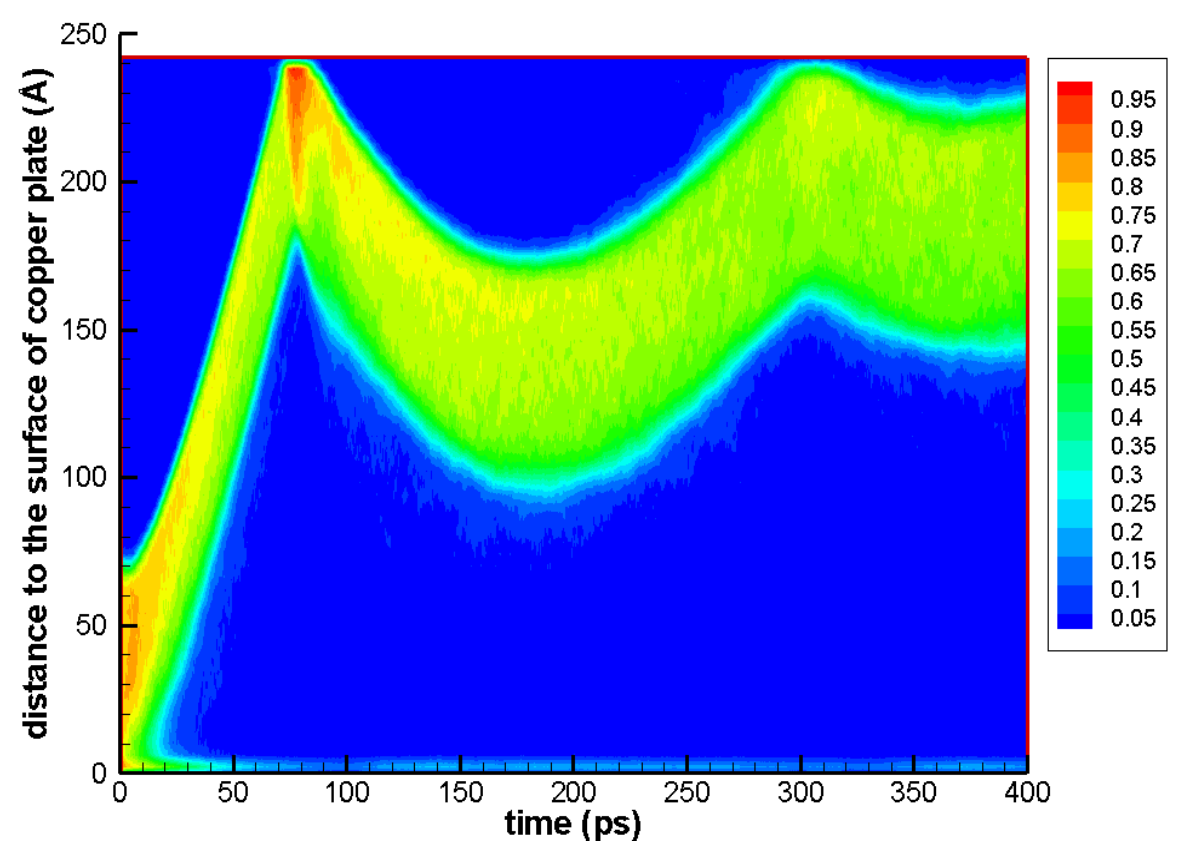

Figure 6-7 Variation of one dimensional spatial density distribution with time

A similar drop also appear in middle bulk water film due to energy conversion to potential energy during mass flux flow to the lower vapor region. For the newly created vapor region, an increase at time $70 \mathrm{ps}$ is caused by absorbing the heat flux continuously entering the vapor region. Because the compression process where the kinetic energy is converted to be potential energy again when bulk liquid hit against the wall, a temperature drop occurs again. Since all the three regions are compressed at this time, so all of them have drops at $80 \mathrm{ps.}$ 


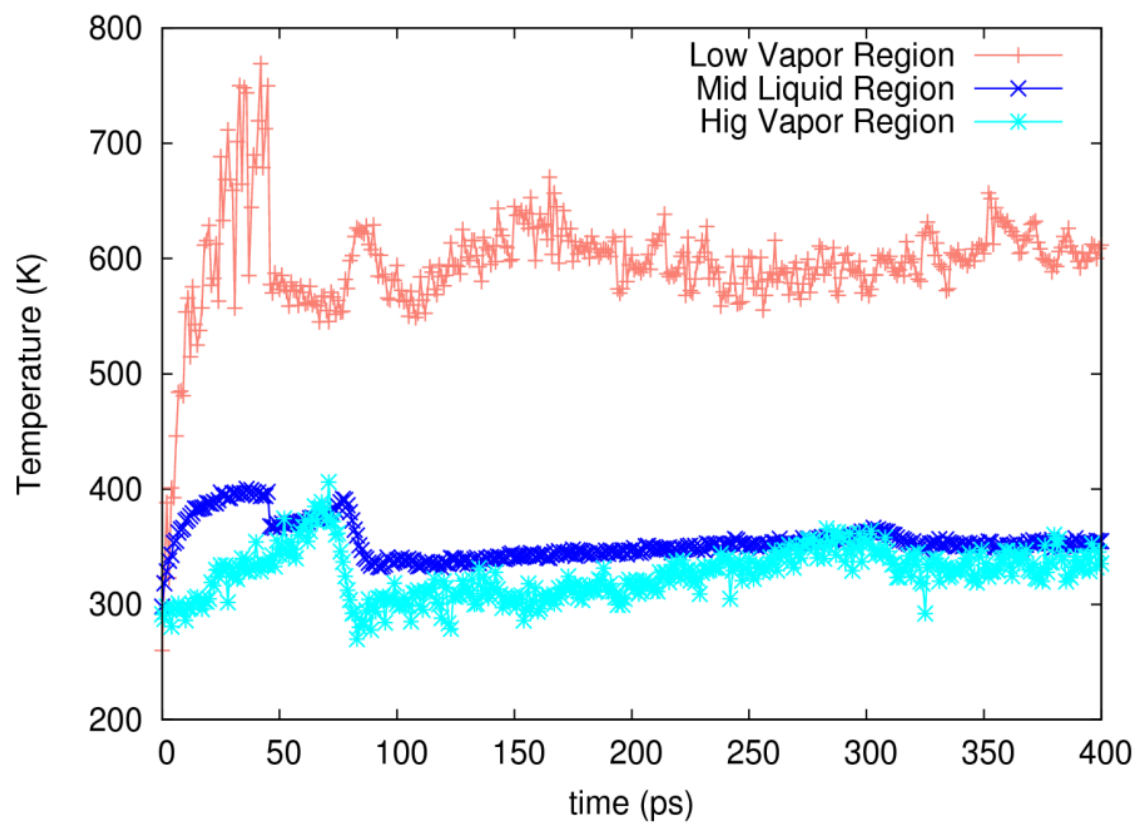

Figure 6-8 Temperature variation in three regions

Similarly, at $300 \mathrm{ps,} \mathrm{an} \mathrm{obvious} \mathrm{temperature} \mathrm{drop} \mathrm{appeared} \mathrm{in} \mathrm{middle} \mathrm{bulk} \mathrm{liquid} \mathrm{and}$ top vapor again due to compression. The temperature in the lower vapor region does not fall because the volume of this region is larger enough to overcome the kinetic energy loss due to compression. In addition, continuously entering heat flux provides enough energy to fill the small amount of the lost energy.

\subsection{Conclusions}

Molecular dynamics simulation is carried out to study the rapid boiling of liquid water film on a hot copper plate. It was found that the bulk liquid water moves like a piston with two vapor regions at bottom and top after phase explosion. The trajectory variation of water molecule's COM value ( $z$-component) indicates that the piston-like motion of the liquid film will finally stop and suspended somewhere close to the top wall. A group of non-vaporization molecular, which has a constant density of $0.2 \mathrm{~g} / \mathrm{cm}^{3}$ and tightly attached to the localized "dry" surface of the plate, is observed no matter how long 
the heat process lasts. It is also found that temperature at each region decreases during the compression process where kinetic energy of molecules is converted to potential energy when water film hits the fixed top wall. It worth to mention that molecular dynamics is highly dependent on the accuracy of potential function that employed, thus different potential functions for water models, copper plate, and interaction between them may lead to uncertainties in results. It is encouraged to study more on how the water models affect the final result in the next stage of research.

\section{Acknowledgement}

Support for this work by the U.S. National Science Foundation under grant number CBET- 1066917 is gratefully acknowledged. 


\section{CHAPTER 7 ATOMISTIC-CONTINUUM HYBRID SIMULATION OF HEAT TRANSFER BETWEEN ARGON FLOW AND COPPER PLATES}

\subsection{Introduction}

Fluid dynamics and heat transfer behaviors in micro fluidics have drawn intensive attentions in the last two decades due to the rapid development of MEMS/NEMS and many other micromechanics applications [104-106]. A better scientific understanding on fundamental mechanism at such small scale will definitely bring a favorable impact on in the foreseeable future. For example, an improved understanding of thermal conductivity from atomic point of view reveal the causes leading to thermal damage of the computer chip which is supposed to be thermally safe under the conventional Fourier law. It is often found that some experimentally measured parameters under micro- spatial/temporal scale, such as heat transfer coefficient at solid-fluid interface and thermal conductivity at solid-solid interface, dramatically disagree with the ones predicted through conventional theory for macro-scale, due to size effect [107]. In order to better understanding the heat transfer mechanism in micro-/nano-scale, numerical simulation is an effective and promising alternative approach.

It is well known that the widely applied three conservation laws can resolve problems for macroscopic scale. However, due to the break-down of continuum assumption, it is also understood that an advanced theory should be developed to remedy the subsistent disadvantage of current conservation laws based simulation tools. Thus, classical molecular dynamics simulations are emerging as another powerful tool to provide 
detailed information on phonon scattering, which further can be used to calculate corresponding thermal properties through certain formula, such as Green-Kubo formulism. A faithful representation of dynamic system should be spatially and temporally large and long enough [107]. As a result, such level of simulation is far beyond the most advanced super computer simulation capability.

As a compromise and meanwhile to take full advantages of both sides, a hybrid simulation scheme that solve three conservation equations in larger domain while resolve atomic trajectory in smaller domain could be a promising approach at the present time. In fact, several effective hybrid simulation methods have been developed to study these particular phenomena caused by the size effect; these methods include atomic finite element method (AFEM) [108], atomistic-smooth particle method [109], and atomisticfinite volume method[110]. The AFEM has advantage of high computational efficiency for solid state problems, and the smooth-particle method is a simulation technique that is still under development [111], which also suffers issues from unclear physical meaning at boundary. A literature survey indicates that atomistic-finite volume method is the most popular hybrid approach among those similar hybrid schemes [112-120].

Since many problems are tangled with fluid flow, finite volume method based hybrid scheme are widely adopted. Yasuda and Yamamoto carried out the hybrid simulations for some element flow of Lennard-Jones liquids and demonstrated the validity of this hybrid simulation scheme. Barsky [121] use this scheme to study dynamic of a single tethered polymer in a solvent. A series of conformational properties of the polymer for various shear rates are obtained. Yasuda, and Yamamoto [122] have demonstrated that the hybrid simulation of molecular dynamic and CFD is able to reach a good consistency as long as 
the mesh size and time step of CFD domain is not too large comparing to the system size and sample during in MD simulation. Wang and He [123] have developed a dynamic coupling model for a hybrid atomistic-continuum in micro- and nano-fluidics. However, most of them primarily emphasize on momentum coupling, while only a few account for energy exchanging at the coupling region. Liu et al. [124] developed computer codes that coupled continuum-atomistic simulator and conducted simulation on heat transfer in micro-/nano- flows. Sun et al. [125] have developed molecular dynamics-continuum hybrid scheme and studied condensation of gas flow in a micro-channel.

An atomic-continuum hybrid simulation of convective heat transfer between argon and two parallel copper plates is carried out. The continuum domain is solved using OpenFOAM [126], while the molecular domain is described using LAMMPS[127]; there is an overlap domain for data exchange between the continuum and molecular domains. This approach is similar to of the recently released CFDEM library [128], but with significant extension. A solver that is fully capable of solving continuum domain and atomic domain through multi-processor super computer system is developed under PISO (Pressure Implicit with Splitting of Operators) solving procedure. It should be mentioned that a reduced unit system is employed in this simulation model. The characteristic dimensions of length, energy, and mass are Lenard-Jones diameter $\sigma$, the minimum value of the potential $\varepsilon$, and the mass $m$ of argon atom, for argon. An asterisk (*) will be used to represent the reduced quantities such as the reduced length $r^{*}=r / \sigma$, and the reduced temperature $T^{*}=T k_{b} / \varepsilon$, the reduced pressure $P^{*}=P \sigma / \varepsilon$, the reduced mass density $\rho^{*}=$ $\rho \sigma^{3} / m$, the reduced time $t^{*}=t\left(\varepsilon / m / \sigma^{2}\right)^{1 / 2}$. All the results will be converted back to the dimensional form for discussion. 


\subsection{Physical Models and Methods}

\subsubsection{Domain Decomposition}

In order to solve the problem, a three dimensional model that involve liquid argon and solid copper wall is created. Since the entire computational domain will be resolved with finite volume method (FVM) and atom-based molecular dynamics, the computational domain is decomposed into three regions: continuum region $(\mathrm{C})$, atomic region $(\mathrm{P})$, and overlapped region $(\mathrm{O})$ between $\mathrm{C}$ and $\mathrm{P}$ regions (see Fig. 1). In the $\mathrm{C}$ region, the fluid flow will be solved with FVM based on classical Navier-Stokes equations, while the $\mathrm{P}$ region will be resolved with classical molecular dynamics with an appropriate potential function. A set of coupling schemes, which will be described in the following section, is adopted to guarantee that momentum and heat flux through specified control layers laying at both the top and bottom of $\mathrm{O}$ region, namely, $\mathrm{P} \rightarrow \mathrm{C}$ and $\mathrm{C} \rightarrow \mathrm{P}$ regions, are continuous. 


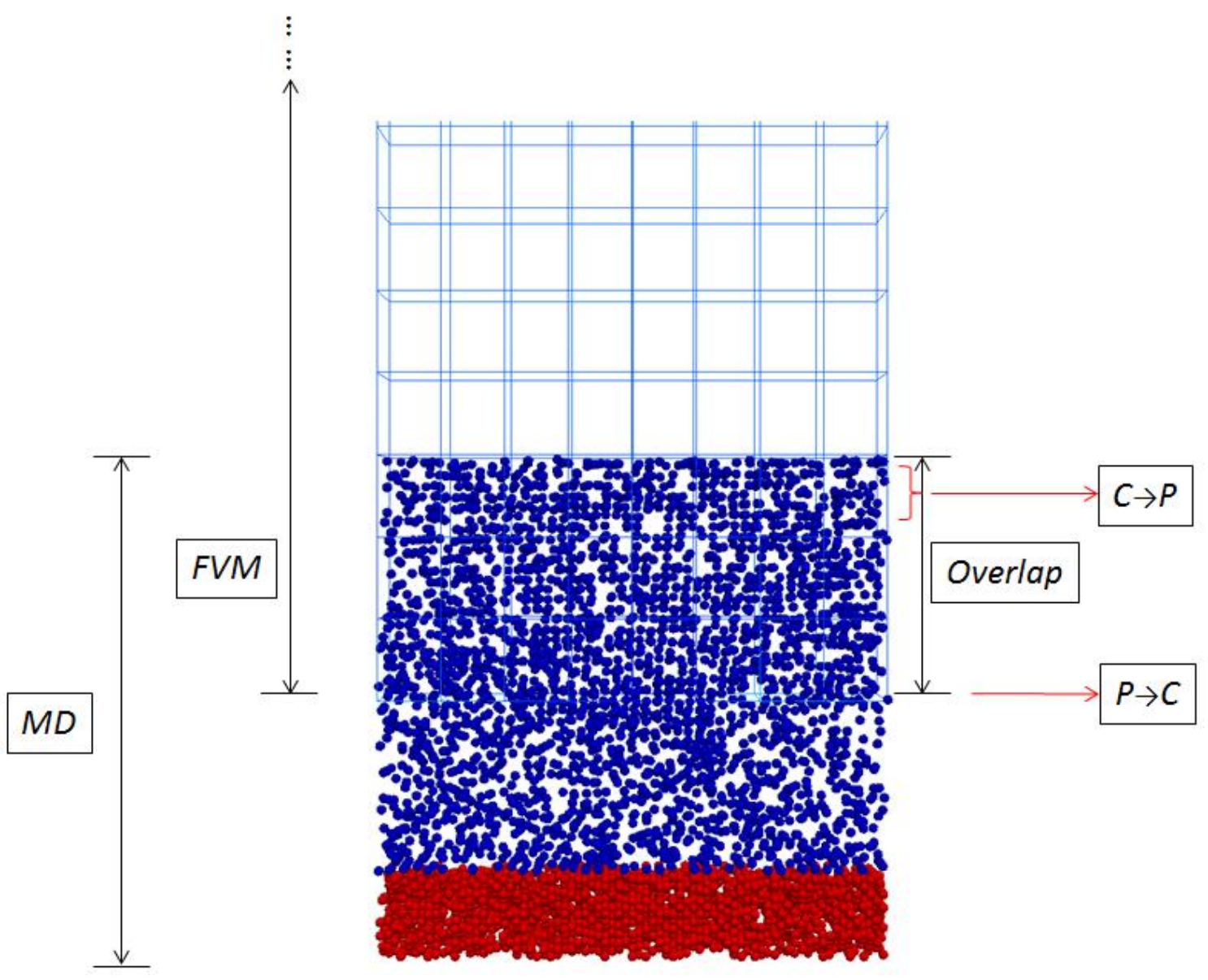

Figure 7-1 Schematic of domain decomposition

\subsubsection{Governing equations for continuum region (C)}

Since the density change during the process is negligible, the argon flow in the continuum region is considered to be incompressible. Meanwhile, the flow is considered to be unsteady, thus any minor affects, such as velocity fluctuation altered through atomic domain during small time-step simulation can be conveyed back and further affects the behaviors in the $\mathrm{C}$ region. The governing equation for $\mathrm{C}$ region can be expressed as follows:

Continuity equation:

$$
\nabla \cdot \mathbf{U}=0
$$


Momentum equation:

$$
\frac{\partial \mathbf{U}}{\partial t}+(\mathbf{U} \cdot \nabla) \mathbf{U}=v \nabla^{2} \mathbf{U}-\frac{1}{\rho} \nabla p
$$

Energy equation:

$$
\frac{\partial \mathrm{T}}{\partial t}+\mathbf{U} \cdot \nabla \mathrm{T}=\frac{\mathrm{k}}{\rho \mathrm{C}_{\mathrm{v}}} \nabla^{2} \mathrm{~T}
$$

It is important to point out that thermal conductivity, dynamic viscosity, and specific heat are treated as constants since the temperature difference within the $\mathrm{C}$ domain is relatively small.

\subsubsection{Potential functions for the atomic region (P)}

In the atomic domain, classical molecular dynamics simulation is adopted to resolve the trajectory evolution of argon atoms. An important assumption for classical molecular dynamics states that the potential energy is a function that only depends on atomic positions. Therefore, an appropriate potential function is essential to describe the interaction among atoms and to govern the dynamic behaviors of atoms in the simulation box. For the interaction between argon atoms, the following commonly accepted modified Lennard-Jones potential, which is able to avoid abrupt energy decrease near cutoff distance, will be used:

$$
\mathrm{V}_{\mathrm{LJ}}\left(\mathrm{r}_{\mathrm{ij}}\right)=4 \varepsilon\left[\left(\frac{\sigma}{\left|\mathrm{r}_{\mathrm{ij}}\right|}\right)^{12}-\left(\frac{\sigma}{\left|\mathrm{r}_{\mathrm{ij}}\right|}\right)^{6}-\left(\frac{\sigma}{\mathrm{r}_{\mathrm{c}}}\right)^{12}+\left(\frac{\sigma}{\mathrm{r}_{\mathrm{c}}}\right)^{6}\right]
$$

where $\varepsilon$ is minimum potential energy and is depth of argon Lennar-Jones potential well, $\boldsymbol{r}_{\mathrm{ij}}$ is vector connecting atoms $\mathrm{i}^{\text {th }}$ and $\mathrm{j}^{\text {th }}$, and $r_{\mathrm{c}}$ is cutoff radius which usually chosen as a length of $3.5 \sigma$.

For the potential function of metallic atoms, the Lennard-Jones type function is not 
well-defined because the contribution from free electrons, which play a key role in thermal transport in most metal, is oversimplified. In fact, as stated in [129], the contribution of free electrons to the thermal conductivity is approximately 100 times more than that of the lattices. Embedded-atom method (EAM) [130] generated potential is a special potential that consider free electron effect on interatomic interaction between ions by introducing an electron density function. And it is fitted to the following mathematical form based on experimental data,

$$
\mathrm{V}_{\text {EAM }}\left(\mathrm{r}_{\mathrm{ij}}\right)=\mathrm{F}_{\mathrm{i}}\left(\sum_{\mathrm{j} \neq \mathrm{i}} \mathrm{f}_{\mathrm{j}}\left(\mathrm{r}_{\mathrm{ij}}\right)\right)+\frac{1}{2} \sum_{\mathrm{j} \neq \mathrm{i}} \varphi\left(\mathrm{r}_{\mathrm{ij}}\right)
$$

where the first term on the right hand side accounts for the electrons contribution due to interaction between electron gas and nuclei. The second term $\varphi$ account for the two-body interaction between the nuclei. Since the classical molecular dynamics assume that electron position relative to nuclei is rigidly fixed (Born-Oppenheimer approximation), which lead to a huge simplification to avoid cumbersome computation for the molecular system, the atomic system can be easily described by solving a set of Newtonian mechanics equations:

$$
m_{\mathrm{i}} \frac{\mathrm{d}^{2} \mathbf{r}_{\mathrm{i}}}{\mathrm{d} t^{2}}=-\sum_{\mathrm{i} \neq \mathrm{j}} \frac{\partial \mathrm{V}\left(\mathbf{r}_{\mathrm{ij}}\right)}{\partial \mathbf{r}_{\mathrm{ij}}}
$$

where $m_{\mathrm{i}}$ is atomic mass of $i^{\text {th }}$ atom, $\boldsymbol{r}$ is the position of atom $i$, and $\mathrm{V}$ is corresponding potential energy.

\subsubsection{Coupling (Overlap) region}

\subsubsection{Momentum coupling}

In the overlap region, a control layer $(\mathrm{C} \rightarrow \mathrm{P})$ is constructed to achieve the momentum consistencies between the atomic and continuum domains. The momentum consistency 
implies that the mean momentum in the molecular region should be equal to the instantaneous macroscopic momentum from continuum region, i.e.:

$$
\left\langle\mathrm{m}_{\mathrm{i}} \mathbf{v}_{\mathrm{i}}\right\rangle=\mathrm{M}_{\mathrm{J}} \mathbf{U}_{\mathrm{J}}
$$

where the left hand side is spatial average of momentum, and the right hand side represents the momentum in the specific control volume.

In order to achieve this momentum consistency, an external force that is proportional to the momentum difference at the same location between the one that from continuum level and the other one from molecular domain will exert on atoms within the control layer (see $\mathrm{C} \rightarrow \mathrm{P}$ layer in Fig. 1). In fact, the velocity in each control volume can be explicitly obtained by solving momentum equations. Another velocity from molecular dynamics within the same control volume can be estimated by:

$$
\mathbf{u}=\left\langle\frac{1}{\mathrm{~N}^{\mathrm{P}-\mathrm{C}}} \sum_{\mathrm{j}=1}^{\mathrm{N}^{\mathrm{P}-\mathrm{C}}} \dot{\mathbf{r}}_{\mathrm{j}}\right\rangle
$$

Thus, the force that will act on each atom in the $\mathrm{C} \rightarrow \mathrm{P}$ region can be estimated through its acceleration:

$$
\ddot{\mathbf{r}}_{i}=\frac{1}{\left(\frac{n}{2}+1\right)}\left(\mathbf{u}_{\mathrm{CFD}}-\left\langle\frac{1}{N^{P-C}} \sum_{j=1}^{N^{P-C}} \dot{\mathbf{r}}_{j}\right\rangle_{n \delta t^{P}}\right)+\left(\frac{\mathbf{f}_{i}}{m}-\left\langle\frac{1}{N^{P-C}} \sum_{j=1}^{N^{P-C}} \frac{\mathbf{f}_{j}}{m}\right\rangle_{n \delta t^{P}}\right)
$$

where the first term reflects the velocity difference and the second term is to adjust the strength of molecules motion. Number $n$ represent coupling interval between two different domains, $\delta t^{\mathrm{P}}$ is the time-step in molecular dynamic simulation, $N^{\mathrm{P}-\mathrm{C}}$ is number molecules within control volume, and the force $f$ is the one derived from potential function. In addition, the last term represents the inherent fluctuation in molecular system 
to accelerate or decelerate molecules. The summation of this term within the control volume is zero [112]. It should be pointed out that even the second term is only expressed as $\boldsymbol{f}_{\mathrm{i}} / m_{i}$, it also obtains good results as reported in some researchers' work [113, 123]. At the same time, the velocity values estimated at the local cell that involve bottom boundary will be assigned as boundary condition of the continuum domain, as shown as $\mathrm{P} \rightarrow \mathrm{C}$ in Fig. 1.

\subsubsection{Temperature coupling}

Similar to the momentum coupling, the temperature field from atomic domain should also match that from the continuum domain. In order to synchronize temperature, each local cell that has atoms in the control layer is connected to a thermal reservoir that holds the temperature of collocated control volume in the continuum domain. Meanwhile, the temperature estimated through kinetic theory will be assigned to continuum domain, through $\mathrm{P} \rightarrow \mathrm{C}$ layer. The temperature from molecular domain is estimated by:

$$
T=\frac{2}{3 k_{B} N^{P-C}}\left\langle\sum_{j=1}^{N^{P-C}} \frac{1}{2} m_{j}\left(\dot{\mathbf{r}}_{j}-\mathbf{u}\right)^{2}\right\rangle
$$

where the velocity $\mathbf{u}$, which is considered as the bulk velocity of a group of molecules located within one control volume, is obtained from the continuum level computation.

The above thermal reservoir that will be connected to the control volume in the control layer is realized by Langevin method [131]. The acceleration of atom $\mathrm{i}$ is calculated from:

$$
\ddot{\mathbf{r}}_{i}=\alpha\left(\dot{\mathbf{r}}_{i}-\frac{1}{N^{P-C}} \sum_{j=1}^{N^{p-C}} \dot{\mathbf{r}}_{j}\right)+\frac{\mathbf{f}_{i}}{m_{i}}+\frac{\mathbf{F}}{m_{i}}
$$

where the first term on the right hand side represents the thermal fluctuation, $\mathbf{f}_{\mathrm{i}}$ is the 
force estimated through potential function, $\mathbf{F}$ is a random force vector that satisfy Gaussian distribution with mean value of 0 and standard deviation of $\left(2 \alpha k_{\mathrm{B}} T / \delta t^{\mathrm{P}}\right)^{1 / 2}$, where $\alpha$ is damping factor and $T$ is target temperature [131]. In this work, the velocity difference is assumed to be immediately eliminated so that the damping factor is set to be 1.0 .

Figure 7-2 shows the computational flowchart which integrate MD evolution into CFD solver based on the solver pisoFoam. Momentum and pressure equations are solved through the PISO algorithm, and temperature field will be resolved afterwards. Classical molecular dynamics simulation will be performed for certain FVM time-step (coupling interval) after reading atoms' velocity and position successively. Then the results from MD will be extracted and transferred back to FVM domain, which will be solved again. The entire simulation ends when the iteration reaches to the total simulation time. 


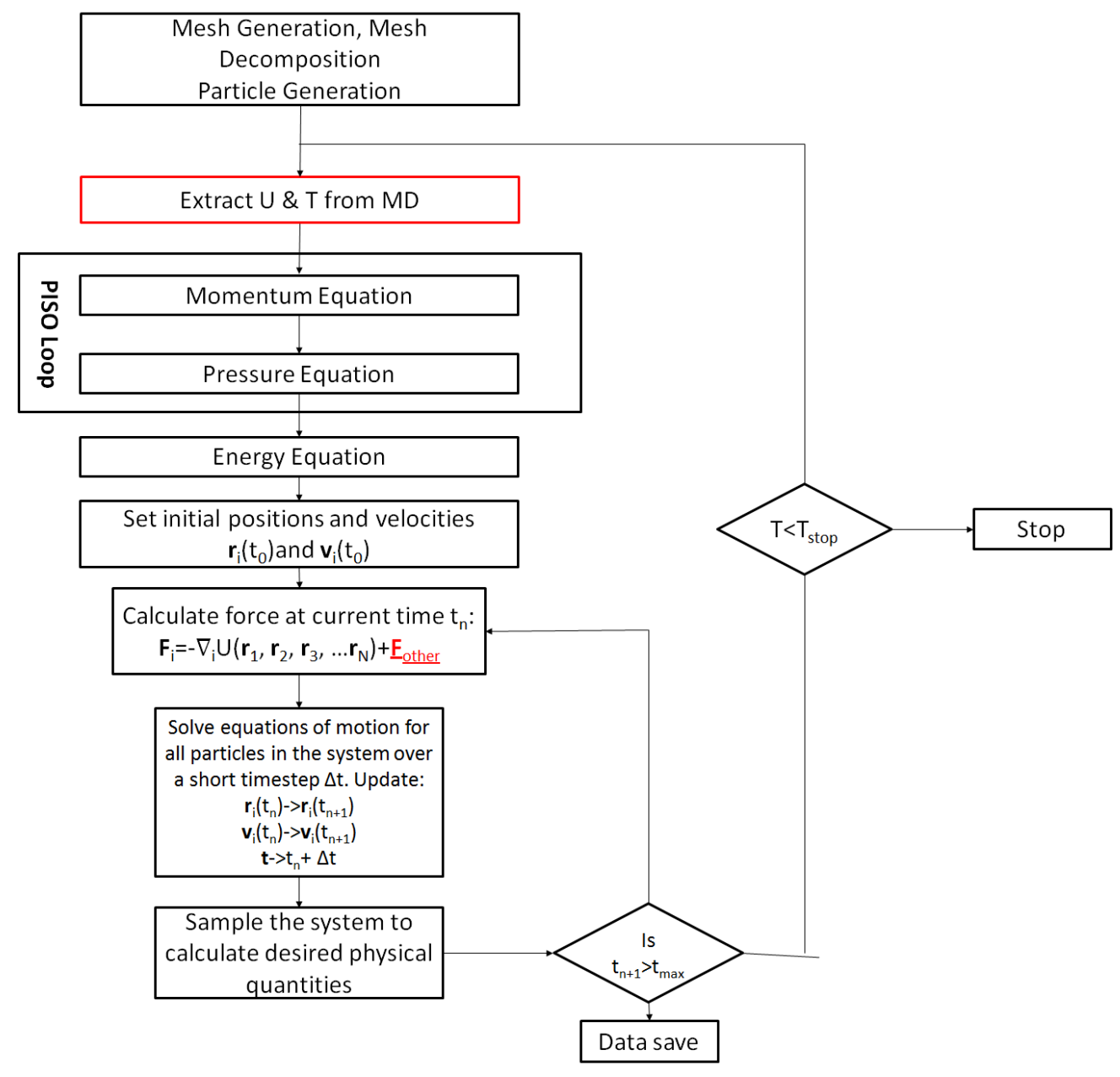

Figure 7-2 Computation flow chart

\subsection{Results and Discussions}

\subsubsection{Code Validation}

In order to validate the implemented schemes, a two-dimensional computational domain which includes both continuum and atomic part is created, as shown in Figure 7-3. It is assumed that the entire domain is filled with liquid argon. For the continuum domain, the fluid has a constant density of $0.83 \mathrm{~m} / \sigma^{3}$, kinematic viscosity of $1.144 \sigma^{2} / \tau$, thermal diffusion of $0.598 \sigma^{2} / \tau$, where the Lennard-Jones parameter $\sigma, \varepsilon$, mass $m$, Boltzmann constant $k_{B}$ are unit, and reduced time $t$ is defined as $\left(\varepsilon / m / \sigma^{2}\right)^{1 / 2}$. And the molecular domain is modeled with 7,390 Lennard-Jones type atoms. It should be pointed 
out that the top edge of molecular domain is considered to be an argon atoms composed wall in order to mimic the interaction between atoms in MD domain and phantom in CFD domain; of course, this treatment can also avoid atom loss. The simulation time step is $0.001 \tau$. In order to validate reliability of momentum and energy coupling, two simple cases, which include pure fluid dynamics and pure heat conduction problem, are solved and compared with published analytical solution.

Couette flow that is driven by a constant moving top wall is a classical problem that is often used for code validation. In order to validate momentum coupling scheme, only the momentum coupling operation will be applied to the overlap domain. In other words, energy transfer procedure, which is also close related to thermal velocity of atoms, will not be activated to alter velocity exchange in this region. However, a thermal reservoir that holds a constant temperature of $1.1 k_{\mathrm{B}} / \varepsilon$ will be connected to the molecular domain, such that the argon atoms will have a desired constant temperature. Figure 3(a) shows that final configuration of this test case, where the molecular domain has both liquid atoms and solid atoms. These solid atoms are used to compose a static wall that mimic physical boundary. And the top wall velocity is set to be $(1.0 \sigma / \tau, 0,0)$. Figure $7-3(\mathrm{~b})$ shows several velocity profiles along the vertical direction at different times, which are $50 \tau, 100 \tau, 200 \tau$, and $2000 \tau$, respectively. It can be seen that the velocity obtained from FVM and MD simulation in the overlap region is consistent with each other at all sampled time, which directly demonstrate that the momentum scheme implement works in a desirably. In fact, the final velocity profile, which is a straight line, also agrees upon the analytical solution [123]. 


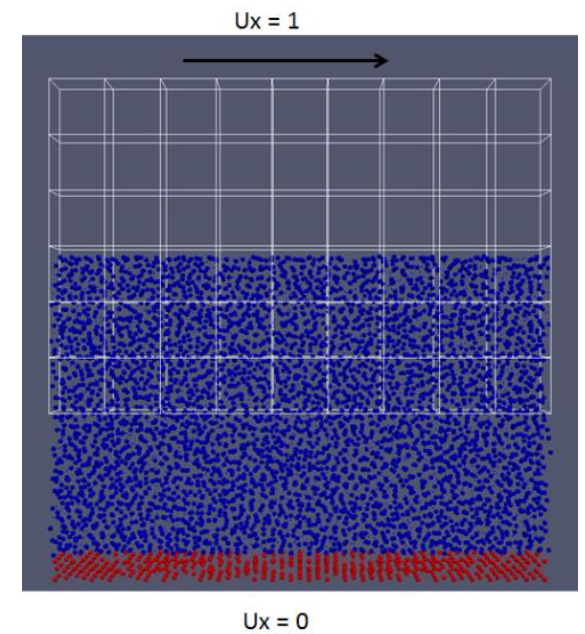

(a)

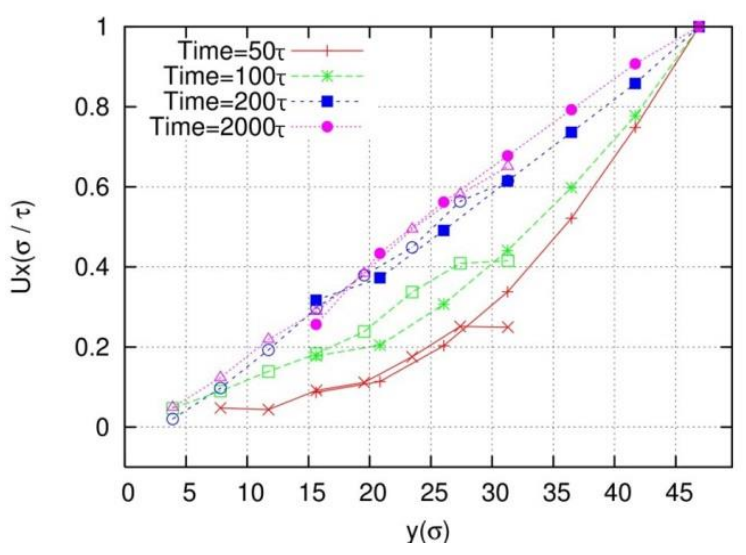

(b)

Figure 7-3 Configuration of test cases and final velocity profiles

To test energy coupling in the overlap domain, heat conduction for both continuum domain and molecular domain is solved. The energy equation in continuum domain is solved to obtain temperature, and only energy coupling is activated through the overlap region this time. The temperature of the top wall of continuum domain is fixed at $1.5 k_{\mathrm{B}} / \varepsilon$ while the bottom wall of molecular domain is set to be $1.1 k_{\mathrm{B}} / \varepsilon$, as shown in Fig. 4(a). Figure 7-4(b) shows temperature profiles at different sample times. It can also be seen that the temperature profile along the vertical line, at different time, is gradually being a flat straight line, which is also agree well with results from other researchers[123]. 


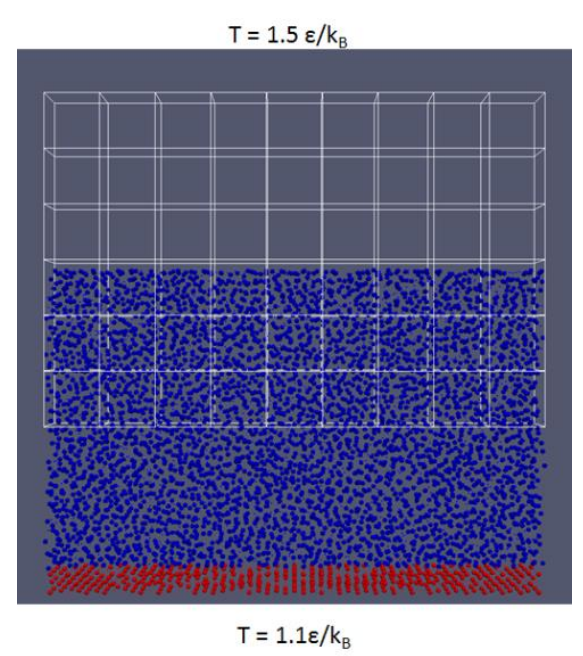

(a)

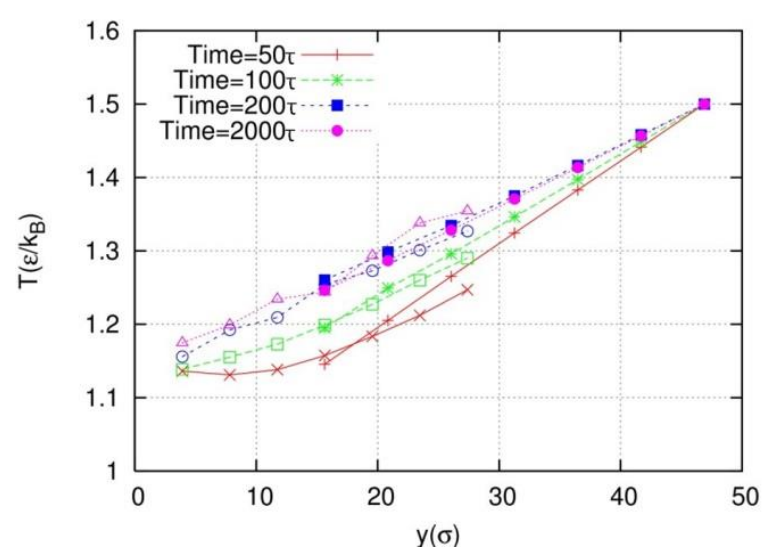

(b)

Figure 7-4 Configuration of test cases and final temperature profiles

\subsubsection{Convection Heat Transfer in Couette Flow}

By following the hypothesis initialized by Tuckerman and Pease [132], the heat transfer coefficient may vary significantly when the size approaching to micro-scale. One observation can be done from the perspective of Nusselt number, whose definition is $\mathrm{Nu}=h D / k$, where $h$ is convective heat transfer coefficient, $D$ is hydraulic diameter, and $k$ is thermal conductivity. It is noticed that $h$ is possible to scaled up to thousand or millions times if the hydraulic diameter $D$ reduce to micro- or nano- size for a fully developed flow in the micro-channel, if the definition of $\mathrm{Nu}$ number is still valid and thermal conductivity is constant at all scales.

In this section, heat transfer coefficient $h$, between argon flow and solid copper surface will be estimated by solving momentum and energy equation simultaneously with finite volume method in continuum domain and simulating the wall-close domain with molecular dynamic approach; momentum and energy are coupled through the overlap region at the same time. A new setup is created to study convective heat transfer 
efficiency by determining heat flux flow across the domain. In comparison with the simple test cases, the layout of the entire computational domain is same, but the atoms in wall domain are replaced with copper atoms. In addition, EAM potential [33] that is able to account for thermal effect contributed by energy carrier of free electron at the very beginning of heating process and also describes the variation of bond strength with coordination is applied to model the interaction between copper atoms. The interaction among argon atoms and the interaction between argon and copper atoms are both described with classical Lennard-Jones-type potential. The parameters in Lennard-Jones potential for argon-argon interaction, $\sigma_{\mathrm{Ar}-\mathrm{Ar}}$ and $\varepsilon_{\mathrm{Ar}-\mathrm{Ar}}$ are simply $\sigma$ and $\varepsilon$ [133] for argon. For interaction between argon and copper atoms, $\sigma_{\mathrm{Ar}-\mathrm{Cu}}$ and $\varepsilon_{\mathrm{Ar}-\mathrm{Cu}}$ are $0.64 \sigma$ and $6.24 \varepsilon$ [134], which is obtained based on geometric mixture manner[135]. There are 7,390 atoms in the atomic domain that holds the same dimension as the cases for validation. In other words, the flow density of atomic domain is consistent with the one in the continuum domain. But the bottom wall is replaced with copper plate that has different lattice constant consistent with density of $3.17 \sigma^{3}$. For the temperature configuration, a Langevin type thermal reservoir is used to control temperature of the copper wall that holds a constant temperature of $1.1 \varepsilon / \mathrm{k}_{\mathrm{b}}$. Meanwhile, the top wall of the continuum domain has a fixed temperature of $1.5 \varepsilon / \mathrm{k}_{\mathrm{b}}$.

For velocity arrangement, a fixed velocity of $(1.0 \sigma / \tau, 0,0)$ is applied to the top wall. It is worth to notice that no jump condition is applied either to temperature or velocity boundary of the top and bottom wall, due to the fact that Knudsen of the system is around 0.01 [136]. For the thermal reservoirs, a damping factor, which determine the interaction frequency with thermal reservoir [127], of 2 is used, instead of 1 after trial and error, and 
they are connected to control volumes within control layer, such that the temperature information can be successfully transmitted to the atomic domain. The coupling interval is 100 time-steps. The time-step is set to be $0.005 \tau$. The cases run for $4000 \tau$ in total, such that the flow in the computational domain is fully developed. In order to achieve a statistically meaningful result, 10 similar cases that have almost the same configurations except for initial atomic positions and velocities are simulated. In addition, in order to measure the heat flux flowing from hot to cold end, a special region between top and bottom wall is created to compute the energy flux when the flow reach to steady state.

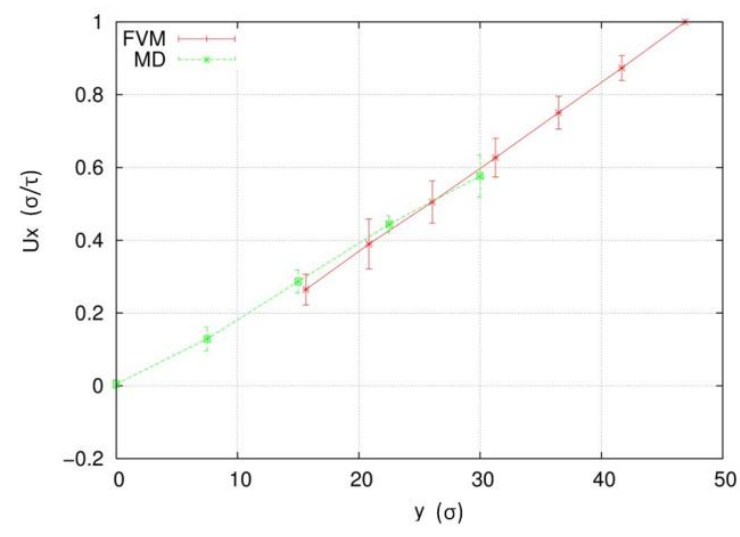

(a)

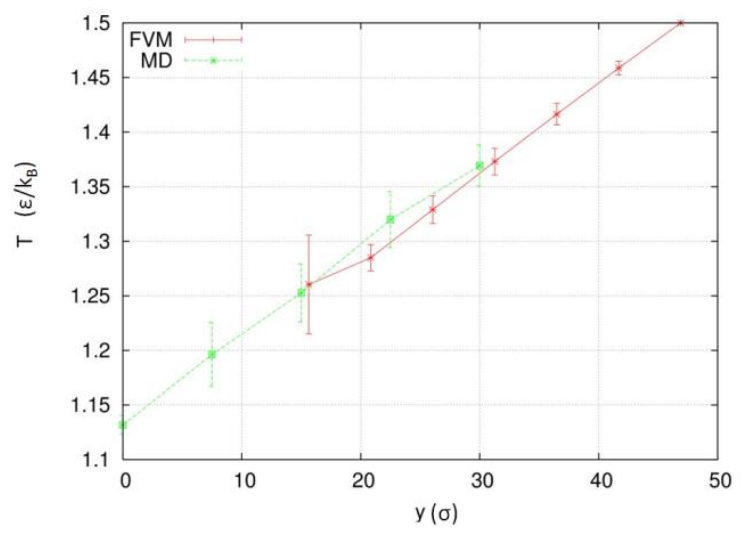

(b)

Figure 7-5 Temperature and velocity distribution along with y axis (Couette flow with

$$
\text { heat transfer) }
$$

Figure 7-5 shows temperature and velocity profiles along the vertical line when the flow is under steady state, where both velocity and temperature profile do not changed with time. It can be observed that the temperature and velocity at coupled zone work well as expected. Both temperature and velocity are consistent with those from the other region, within an acceptable statistical error.

Finally, the heat transfer coefficient between argon flow and copper wall is estimated 
through equation as following,

$$
h=\frac{Q}{A \Delta t \Delta T}
$$

where $Q$ it the total energy passes through copper wall, $\Delta t$ is the time period that this amount of energy pass through, $\Delta T$ is the temperature difference between the copper wall and bulk temperature of argon flow, and $A$ is the surface area of the wall that expose to the argon flow. From the atomic perspective, the heat flux can be estimated through [137]:

$$
q=\frac{Q}{A \Delta t}=\frac{1}{V}\left[\sum_{i} e_{i} \mathbf{v}_{i}-\sum_{i} S_{i} \mathbf{v}_{i}\right]=\frac{1}{V}\left[\sum_{i} e_{i} \mathbf{v}_{i}+\sum_{i}\left(\mathbf{f}_{i j} \cdot \mathbf{v}_{j}\right) \mathbf{r}_{i j}\right]
$$

where $e$ represents internal energy of atom, $\boldsymbol{v}_{\boldsymbol{i}}$ is velocity of atom, $\mathbf{f}_{\mathrm{ij}}$ is pair-wise force between atoms, and $\mathbf{r}_{\mathrm{ij}}$ is relative position between atoms $\mathrm{i}$ and $\mathrm{j}$.

Figure 7-6 shows the real-time variation of heat flux passing through preset region in the atomistic domain. It can be seen that the recorded heat flux fluctuated within a certain and small range. It worth to point out that the sign change of heat flux is mainly caused by large noise and relatively small temperature gradient across the entire atomistic computational domain. It is expected that the relative noise level will significantly decrease with higher temperature gradient. In this simulation work, however, it is not appropriate to impose a large temperature gradient due to a small temperature difference between melting and boiling points of the argon. For this reason, the computational domain is limited to current domain sizes. As shown in Figure 7-6, a clear trend shows that the heat flux is negative though some values are above zero due to noise. The heat flux, which is $-0.086 \varepsilon / \sigma^{2} \tau$, adopted to compute heat transfer coefficient is an averaged value over the latest period of $1000 \tau$, based on 10 cases. It is found that the heat transfer 
coefficient between argon flow and copper plate is $0.25 \mathrm{~kb} / \sigma^{2} \tau\left(1.21 \times 10^{7} \mathrm{~W} / \mathrm{m}^{2} \mathrm{~K}\right)$, which is larger but still in the same order magnitude with the one $\left(0.83 \times 10^{7} \mathrm{~W} / \mathrm{m}^{2} \mathrm{~K}\right.$, with $\mathrm{Nu}=1.38, k=96.4 \mathrm{~mW} / \mathrm{m} \mathrm{K}$ [35]) predicted based on the hypothesis in reference [36], where the expected value may increase significantly if the definition of Nusselt is assumed to be valid and thermal conductivity is constant at micro-scale.

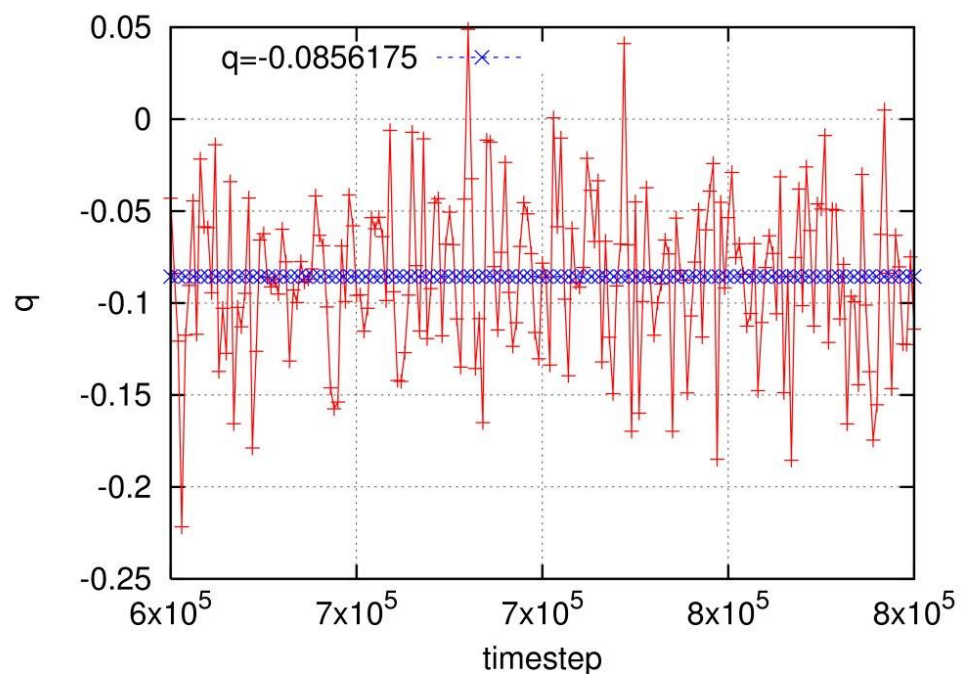

Figure 7-6 Heat flux variation along with simulation time-step

\subsection{Conclusions}

In this work, atomistic-continuum hybrid simulation of heat transfer between argon flow and copper plate is carried out, based on a general solver that is developed based on OpenFOAM and LAMMPS. The validity of this coupling scheme is tested through a Couette flow case and a heat conduction case. The solver achieves satisfactory agreement with the existing results for testing cases, which mean momentum and energy successfully meet continuous requirement at the overlap region. Finally, heat transfer coefficient between flowing argon and solid copper plate is studied within this frame work. Both velocity and temperature are coupled at the overlap region simultaneously, and reach to stable profiles in vertical direction. Then heat flux flowing across the sample 
region is measured in order to compute heat transfer efficiency. It is found that the heat transfer coefficient is larger but still in same order magnitude with the one predicted based on the hypothesis in reference [36]. Further investigation is desired to explore heat transfer coefficient for micro-channel with other flow medium (such as water), which has

supportive experimental data, and also allow to impose high temperature to the top and bottom walls.

\section{Acknowledgement}

Support for this work by the U.S. National Science Foundation under grant number CBET- 1066917 is gratefully acknowledged. 


\section{CHAPTER 8 CONCLUSIONS AND FUTURE WORK}

\subsection{Conclusions}

In this dissertation, micro-scale heat transfer phenomena on water is conducted with molecular dynamics and hybrid approach. From Chapter 2, it is found that five site models is more capable to reproduce thermal conductivity and shear viscosity, while three sites models is more preferable for computing specific heat, when the system is at a temperature of $298 \mathrm{~K}$ and $318 \mathrm{~K}$. The Chapter 3 widen the temperature range based on the work in Chapter 2. It shows that five site model is able to yield thermal conductivity values quite well when the temperature of liquid water is at the range from $283 \mathrm{~K}$ to $363 \mathrm{~K}$. However, for shear viscosity computation, it is found that three and four site models start giving a better prediction when the liquid water temperature is higher than $320 \mathrm{~K}$, below which five site model still have the best performance among all. The dynamic behavior of a nano bubble growth and annihilation is simulated in Chapter 4 , it is found that Reyleigh-Plesset equation that is derived from macroscopic point of view fail to reproduce radius variation during the entire process. A simulation box that involve a static water droplet and CNT composed plate is created to study wetting process when two parallel CNTs are immersed in the liquid water and contact angle of a single droplet sitting on the plate, as presented in Chapter 5. The result shows that when the separate distance between two CNTs is larger than $13 \AA$, the gap between two CNTs will be wetted. It is also found that the contact angle of nano water droplet have the maximum value when the separate distance is around $13 \AA$. In Chapter 6, a rapid boiling process is studied by placing a cold water thin film on a $1000 \mathrm{~K}$ hot copper plate. The simulation 
shows that the thin film moves rapidly when phase change in the water film occur. A hotgas like zone is observed no matter how hot the plate is. This phenomena is be caused by van der Waals force between vapor water molecules and copper atoms. From the macroscopic point of view, a similar phenomenon caused by disjoining pressure between two parallel plates also exist. In Chapter 7, a hybrid simulation method which combine molecular dynamics and finite volume method is presented based on three open-source code, including OpenFOAM, LAMMPS, and CFDEM. Heat transfer coefficient calculated by this method gives a value around $1.2 \times 107 \mathrm{~W} / \mathrm{m}^{2} \mathrm{~K}$, which is close to the one predicted from analytical solution $\left(1.46 \times 107 \mathrm{~W} / \mathrm{m}^{2} \mathrm{~K}\right.$, after correction).

\subsection{Future work}

In comparison with liquid water, argon fluid flow is not realistic, it is desired to replace argon molecules with various water molecules under the frame work in Chapter 7 in the near future.

Computational efficiency improvement on current CFD-MD coupling library can be done by introducing a special region model, which is able to identify those atoms in the overlap region. Such that, the computational efficiency could be dramatically improved.

In addition, only eight rigid water molecules are selected for examining capability of simulating properties or any other application in a number of non-equilibrium physical processes, it is also urgent to test all the rest water molecule under the same framework done shown before, especially the PPC water model which has simple structure (definitely save huge computational cost).

If time is allowed, it is also desired to develop a many-body potential for current water models, which contribute $14.5 \%$ to internal energy of single water molecule. 


\section{REFERENCES}

[1] Tzou, D. Y. Macro- to Microscale Heat Transfer: The Lagging Behavior. Bristol, PA: Taylor \& Francis; 1996.

[2] Compte, A., Metzler, R., "The Generalized Cattaneo Equation for the Description of Anomalous Transport Processes," Journal of Physics A: Mathematical and GeneralEmail alert RSS feed, 1997; 30(21): p. 13.

[3] Tzou, D. Y., "A Unified Field Approach for Heat Conduction from Macro- to Micro-Scales," Journal of Heat Transfer, 1995; 117(1): p. 9.

[4] Chen, J. K., Tzou, D. Y., Beraun, J. E., "A Semiclassical Two-Temperature Model for Ultrafast Laser Heating," International Journal of Heat and Mass Transfer, 2006; 49(1-2): p. 10.

[5] Mayadas, A. F., Shatzkes, M., Janak, J. F., "Electrical Resistivity Model for Polycrystalline Flims: The Case of Specular Reflection at External Surface," Applied Physics Letters, 1969; 14(11): p. 3.

[6] Alder, B. J., Wainwright, T. E., "Phase Transistion for a Hard Sphere System," Journal of Chemical Physics, 1957; 27(5).

[7] Gilson, J. B., Goland, A. N., Milgram, M., Vineyard, G. H., "Dynamics of Radiation Damage," Phys Rev, 1960; 120.

[8] Rahman, A., "Correction in the Motion of Atoms in Liquid Argon," Phys Rev, 1964; 136.

[9] Verlet, L., "Computer "Experiments" on Classical Fluids. I. Thermodynamical Properties of Lennard-Jones Molecules," Physcial Review, 1967; 159: p. 6. 
[10] Stillinger, F. H., Rahman, A., "Improved Simulation of Liquid Water by Molecular Dynamics," Journal of Chemical Physics, 1974; 60(4): p. 13.

[11] McCammon, J. A., Gelin, B.R., Karplus, M. , "Dynamics of Folded Proteins," Nature, 1977; 267: p. 6.

[12] McQuarrie, D. A. Statistical Mechanics. Sausalito, California: Universitiy Science Books; 2000.

[13] Hodges, M. P., Stone, A. J., Xantheas, S. S., " Contribution of Many-Body Terms to the Energy for Small Water Clusters: A Comparison of Ab Initio Calculations and Accurate Model Potentials," Journal of Physical Chemistry A, 1997; 101: p. 6.

[14] Chaplin, M., "Water Structure and Science," 2013.

[15] Guillot, B., "A Reappraisal of What We Have Learnt During Three Decades of Computer Simulations on Water," Journal of Molecular Liquids, 2002; 101.

[16] Caleman, C., "Water Models in Computer Simulations," Journal of Chemical Physics, 2007; 221.

[17] Abascal, J. L. F., Vega, C. , "A General Purpose Model for the Condensed Phases of Water: Tip4p/2005," Journal of Chemical Physics, 2005; 123: p. 234505.

[18] Horn, H. W., Swope, W. C., Pitera, J. W. , "Development of an Improved FourSite Water Model for Biomolecular Simulations: Tip4p-Ew," Journal of Chemical Physics, 2004; 120(20): p. 13.

[19] Rick, S. W., "A Reoptimization of the Five-Site Water Potential (Tip5p) for Use with Ewald Sums," Journal of Chemical Physics, 2004; 120(13): p. 9. 
[20] Bizzarri, A. R., Cannistraro, S. , "Molecular Dynamics of Water at the ProteinSolvent Interface," Journal of Physical Chemistry B, 2002; 106: p. 7.

[21] Jorgensen, W. L., Chandrasekhar, J., Madura, J. D., "Comparison of Simple Potential Functions for Simulating Liquid Water," Journal of Chemical Physics, 1983; 79: p. 9 .

[22] Mahoney, M. W., "A Five-Site Model for Liquid Water and the Reproduction of the Density Anomaly by Rigid, Nonpolarizable Potential Function," Journal of Chemical Physics, 2000; 112(20): p. 13.

[23] Berendsen, H. J. C., Grigera, J. R., Straatsma, T. P., "The Missing Term in Effective Pair Potential," Journal of Physical Chemistry, 1987; 91: p. 3.

[24] González, M. A., Abascal, J. L. F., "The Shear Viscosity of Rigid Water Models," Journal of Chemical Physics, 2010; 132.

[25] Haile, J. M. Molecular Dynamics Simulation, Elementary Methods. Chichester: Wiley; 1992.

[26] Müller-Plathe, F., "A Simple Nonequilibrium Molecular Dynamics Method for Calculating the Thermal Conductivity," Journal of Chemical Physics, 1997; 106.

[27] Zhang, M., Lussetti, E., Souza, L. E. S. D., Müller-Plathe, F., "Thermal Conductivities of Molecular Liquids by Reverse Nonequilibrium Molecular Dynamics," Journal of Physical Chemistry B, 2005; 109: p. 8.

[28] Bedrov, D., Smith, G. D., "Thermal Conductivity of Molecular Fluids from Molecular Dynamics Simulations: Application of a New Imposed-Flux Method," Journal of Chemical Physics, 2000; 113(18): p. 5. 
[29] Forsblom, M., Grimvall, G., "Heat Capacity of Liquid Al: Molecular Dynamics Simulation," Physics Review B, 2005; 72: p. 132204.

[30] Berendsen, H. J. C., Postma, J. P. M., Van Gunsteren, W.F., Hermans, J. . In Intermolecular Forces. Dordrecht: D. Reidel Publishing Company; 1981.

[31] Robinson, G. W., Zhu, S. B. , Singh, S., Evans, M. W. . Water in Biology, Chemistry and Physics: Experimental Overviews and Computational Methodologies. Singapore: World Scientific; 1996.

[32] Hockney, R. W., Eastwood, J. W. . Computer Simulation Using Particles. New York: Taylor \& Francis; 1998.

[33] Ryckaert, J. P., Ciccotti, G., Berendsen, H. J. C., , "Numerical Integration of the Cartesian Equation of Motion of a System with Constraints: Molecular Dynamics of N-Alkanes," Journal of Computational Physics, 1997; 23: p. 5.

[34] Müller-Plathe, F., "Reversing the Pertubation in Non-Equiliabrium Molecular Dynamics: An Easy Way to Calculate the Shear Viscosity of Fluids," Physical Review E, 1999; 59(5): p. 5.

[35] Hünenberger, P. H., "Thermostat Algorithms for Molecular Dynamics Simulations," Advance in Polymer Science, 2005; 173: p. 45.

[36] Plimpton, S. J., "Fast Parallel Algorithms for Short-Range Molecular Dynamics," Journal of Computation Physics, 1995; 117: p. 19.

[37] Haynes, W. M. Handbook of Chemistry and Physics, 91st Ed. New York: Taylor \& Francis. 
[38] Lee, H. S., Tuckerman, M. E., "Dynamical Properties of Liquid Water from Ab Initio Molecular Dynamics Performed in the Complete Basis Set Limit," Journal of Chemical Physics, 2007; 126: p. 164501.

[39] Chialvo, A. A., Houssa, M., Cummings, P. T., "Molecular Dynamics Study of the Structure and Thermophysical Properties of Model Si Clathrate Hydrates," Journal of Physical Chemistry B, 2002; 106(2): p. 10.

[40] Bertolini, D., "Thermal Conductivity of Water: Molecular Dynamics and Generalized Hydrodynamics Results," Physical Review E, 1997; 56(4): p. 17.

[41] Mark P., N. L., "Structure and Dynamics of the Tip3p, Spc, and Spc/E Water Models at 298k," Journal of Physical Chemistry A, 2001; 105: p. 7.

[42] Bordat, P., Müller-Plathe, F. , "The Shear Viscosity of Molecular Fluids: A Calculation by Reverse Nonequilibrium Molecular Dynamics," Journal of Chemical Physics, 2002; 116(18): p. 8.

[43] Mao, Y. J., Zhang, Y. W., "Thermal Conductivity, Shear Viscosity and Specific Heat of Rigid Water Models," Chem Phys Lett, 2012; 542: p. 5.

[44] Berendesen, H. J. C., Van Gunsteren, W. F. . Molecular Liquids: Dynamics and Interactions, Nato Asi C 135: Reidel: Dordrecht; 1984.

[45] Moholkar, V. S., Pandit, A. B. , "Bubble Behavior in Hydrodynamic Cavitation: Effect on Turbulence," AIChE Journal, 1997; 43(6): p. 8.

[46] Gerstne, r., "Bubble Nucleation: Stout Fizz-Ics," Nature Physics, 2011; 7.

[47] Rosato, D. V., Schott, N. R., Rosato, M. G. . Plastics Engineering, Manufacturing \& Data Handbook. Norwell, Massachusetts, USA: Kluwer; 2001. 
[48] Park, S., Weng, J. G. , Tien, C. L. , "Cavitation and Bubble Nucleation Using Molecular Dynamics Simulation," Microscale Thermophysical Engineering, 2000; 4(3): p. 15.

[49] Wu, Y. W., Pan, C. , "A Molecular Dynamics Simulation of Bubble Nucleation in Homogeneous Liquid under Heating with Constant Mean Negative Pressure," Microscale Thermophysical Engineering, 2003; 7(2): p. 5.

[50] Tsuda, S. I., Tokumasu, T. , Kamijo, K. , "A Molecular Dynamics Study of Bubble Nucleation in Liquid Oxygen with Impurities," Heat Transfer-Asian Research, 2005; 34(7): p. 13.

[51] Zhai, M., Zhang, R. Z., Li , Q., Shen, C., "Molecular Dynamics Simulation of Bubble Nucleation in Carbon Dioxide/Hexadecane Solution as an Analog to Foaming of Polyethylene by Super Critical Carbon Dioxide," Journal of Plastic Film and Sheeting, 2011; 27(1-2): p. 9.

[52] Lugli, F., Zerbetto, F. , "Molecular Dynamics of Nanobubbles' Collapse in Ionic Solutions," ChemPhysChem, 2007; 8(1): p. 3.

[53] Pu, J., Yang, L., Zu, X. T., Gao, F. , "A Molecular Dynamics Study of Helium Bubble Stability During High-Energy Displacement Cascades in A-Iron," Physica $B, 2007 ; 398:$ p. 6.

[54] Insepov, Z., Hassanein, A. , "Molecular Dynamics Simulation of Li Surface Erosion and Bubble Formation," Journal of Nuclear Materials, 2005; 337-339 p. 5. 
[55] Nagayama, G., Tsuruta, T., Cheng, P. , "Molecular Dynamics Simulation on Bubble Formation in a Nanochannel," International Journal of Heat and Mass Transfer, 2006; 49: p. 7.

[56] Okumura, H., Ito, N., "Nonequilibrium Molecular Dynamics Simulations of a Bubble," Physics Review E, 2003; 67(4).

[57] Jorgensen, W. L., Chandrasekhar, J., Madura, J. D., "Comparison of Simple Potential Functions for Simulating Liquid Water," Journal of Chemical Physics, 1983; 79: p. 10.

[58] Moody, M. P., Attard, P. , "Curvature Dependent Surface Tension from a Simulation of a Cavity in a Lennard-Jones Liquid Close to Coexistence," Journal of Chemical Physics, 2001; 115(9): p. 11.

[59] Shi, B., Sinha, S., Dhir, V.K., "Molecular Dynamics Simulation of the Density and Surface Tension of Water by Particle-Particle Particle-Mesh Method," Journal of Chemical Physics, 2006; 124.

[60] MacKerll, A. D. J., Bashford, D., Bellott, M., Dunbrack, R.L. Jr., Evanseck, J.D., Field, M.J., Fischer, S., Gao, J., Guo, H., Ha, S., Joseph-MaCarthy, D., Kuchnir, L., Kuczera, K., Lau, F.T.K., Mattos, C., Michnick, S., Ngo, T., Nguyen, D.T., Prodhom, B., Reiher, III, W.E., Roux, B., Schlenkrich, M., Smith, J.C., Stote, R., Straub, J., Watanabe, M., Wiórkiewicz-Kuczera, J., Yin, D. , Karplus, M., "AllAtom Empirical Potential for Molecular Modeling and Dynamics Studies of Proteins," Journal of Physical Chemistry B, 1998; 102: p. 31.

[61] Humphrey, W., Dalke, A., Schulten, K., "Vmd-Visual Molecular Dynamics," Journal of Molecular Graphics, 1996; 14: p. 6. 
[62] Heggie, M. I., Latham, C.D., Maynard, S. C. P., Jones, R., "Cooperative Polarisation in Ice Ih and the Unusual Strength of the Hydrogen Bond," Chemical Physics Letters, 1996; 249(5-6): p. 6.

[63] Iijima, S., "Helical Microtubules of Graphitic Carbon," Lett Nat, 1991; 354: p. 3.

[64] Harris, P. J. F. Carbon Nanotubes and Related Structures. Cambridge: Cambridge University Press; 1999.

[65] Wang, J., "Carbon-Nanotube Based Electrochemical Biosensor: A Review. ," Electroanalysis, 2005; 17(1): p. 8.

[66] Korrdas, K., Toth,G., Moilanen, P., Kumpumaki, M., Vahakangas, J. , Uusimaki, A., Vajtai, R., Ajayan, P. M. , "Chip Cooling with Integrated Carbon Nanotube Microfin Architectures," Appl Phys Lett, 2007; 90.

[67] Sun, T. L., Wang, G .J., Liu, H. , Feng, L., Jiang ,L., Zhu, D. B. , "Control over the Wettability of an Aligned Carbon Nanotube Film," J Am Chem Soc, 2003; 125: p. 2.

[68] Jarvis, S. P., Uchihashi, T., Ishida, T. , Tokumoto, H., Nakayama, Y. , "Local Solvation Shell Measurement in Water Using a Carbon Nanotube Probe," J Phys Chem B, 2000; 104: p. 4.

[69] Cai, D., Ren, L., Zhao, H. Z., Xu, C.J., Zhang, L., Yu, Y., Wang, H. Z., Lan, Y. C., Roberts, M. F., Chuang, J. H. , Naughton, M. J., Ren, Z. F. , Chiles, T.C., "A Molecular-Imprint Nanosensor for Ultrasensitive Detection of Proteins," Nat Nanotechnol; 5: p. 5.

[70] Sergi, D., Scocchi, Ortona, G., A. , "Molecular Dynamics Simulations of the Contact Angle between Water Droplets and Graphite Surface," eprint, 2012. 
[71] Rafiee, J., Mi, X., Gullapalli, H., Thomas, A. V., Yavari, F., Shi, Y. F, Ajayan, P. M., Koratkar, N. A., "Wetting Transparency of Graphene," Nat Mater, 2012; 11: p. 6.

[72] Journet, C., Moulinet, S., Ybert, C., Purcell, S.T., Bocquet, L., "Contact Angle Measurements on Superhydrophobic Carbon Nanotube Forests: Effect of Fluid Pressure. ," Europhys Lett, 2005; 71(1): p. 6.

[73] Men, X. H., Zhang, Z.Z. , Song, H. J. , Wang, K. , Jiang, W. , "Fabrication of Superhydrophobic Surface with Poly(Furfurl Alcohol)/Multi-Walled Carbon Nanotubes Composites," Appl Surf Sci, 2008; 254.

[74] Werder, T., Walther, J. H., Jaffe, R. L., Halicioglu, T., Noca, F. , Koumoutsakos, P. , "Molecular Dynamics Simulation of Contact Angles of Water Droplets in Carbon Nanotubes," Nano Lett, 2001; 1(12): p. 6.

[75] Alexiadis, A., Kassinos, S. , "Molecular Simulation of Water in Carbon Nanotubes," Chem Rev, 2008; 108: p. 21.

[76] Tzeng, Y., Huang, T.S. ,Chen, Y.C. , Liu, C. , Liu, Y. K. , "Hydration Properties of Carbon Nanotubes and Their Effects on Electrical and Biosensor Applications," New Diam Front Carbon Technol, 2004; 14.

[77] Huang, L., Lau, S. P., Yang, H. Y., Leong, E. S. P., Yu, S. F. , "Stable Superhydrophobic Surface Via Carbon Nanotubes Coated with a Zno Thin Film," J Phys Chem B, 2005; 109: p. 3.

[78] Li, J. L., Wang, L. J., Jiang, W. , "Super-Hydrophobic Surface of Bulk Carbon Nanotubes Compacted by Spark Plasma Sintering Followed by Modification with Polytetrofluorethylene," Carbon, 2010; 48: p. 30. 
[79] Aria, A. I., Gharib, M. , "Bouncing Water Droplet on a Superhydrophobic Carbon Nanotube Array," eprint, 2010.

[80] Niu, S. Q., Tan, M.L., Ichiye, T. , "The Large Quadrupole of Water Molecules," $J$ Chem Phys, 2011; 134.

[81] Darden, T., York, D., Pedersen, L., "Particle Mesh Ewald: An N·Log(N) Method for Ewald Sums in Large Systems," Journal of Chemical Physics, 1993; 98(12): p. 4.

[82] Walther, J. H., Jaffe, R., Halicioglu, T., Koumoutsakos, P., "Carbon Nanotubes in Water: Structural Characteristics and Energetics," J Phys Chem B, 2001; 105: p. 8.

[83] Hansen, F. Y., Bruch, L. W. , "Molecular Dynamics Study of the Dynamical Excitations in Commensurate Monolayer Films of Nitrogen Molecules on Graphite: A Test of the Corrugation in the Nitrogen-Graphite Potential," Phys Rev $B, 1995 ;$ 51(4): p. 22.

[84] Thorn, K. S., "Multipole Expansions of Gravitional Radiation," Reviews of Modern Physics, 1980; 52(2): p. 41.

[85] Wallqvist, A., Berne, B.J. , "Computer Simulation of Hydrophobic Hydration Forces on Stacked Plates at Short Range," J Phys Chem B, 1995; 99: p. 7.

[86] Cruz-Chu E. R., A., A., Schulten, K. , "Water-Silica Force Field for Simulating Nanodevices," J Phys Chem B, 2006; 110: pp. 21497-21508.

[87] Park, S. H., Carignano, M. A., Nap, R. J., Szleifer, I. , "Hydrophobic-Induced Surface Reorganization: Molecular Dynamics Simulations of Water Nanodroplets on Perfluorocarbon Self-Assembled Monolayers," Soft Matter, 2010; 6(8): p. 11.

[88] Galassi, M., et. al. Gnu Scientific Library Reference Manual. 
[89] Faghri, A., Zhang, Yu Wen. Transport Phenomena in Multiphase Systems. Burlington, MA, USA: Elsevier; 2010.

[90] Takamizawa, A., Kajimoto, S., Hobley, J., Hatanaka, K., Ohta, K., Fukumura, H., "Explosive Boiling of Water after Pulsed Ir Laser Heating, Physical Chemistry Chemical Physics," Physical Chemistry Chemical Physics, 2003; 5: p. 8.

[91] She, M., Kim, D., and Grigoropoulos, C. P., "Liquid-Assisted Pulsed Laser Cleaning Using near-Infrared and Ultraviolet Radiation," Journal of Applied Physics, 1999; 86(11): p. 5.

[92] Xu, X. F., "Phase Explosion and Its Time Lag in Nanosecond Laser Ablation," Applied Surface Science, 2002; 197: p. 6.

[93] Kudryashov, S. I., Allen, S. D., "Submicrosecond Dynamics of Water Explosive Boiling and Lift-Off from Laser-Heated Silicon Surfaces," Journal of Applied Physics, 2006; 100.

[94] Dou, Y. S., Zhigilei, L. V., Winograd, N., Garrison, B., "Explosive Boiling of Water Films Adjacent to Heated Surfaces: A Microscopic Description," Journal of Physical Chemistry, 2001; 105: p. 8.

[95] Gu, X., Urbassek, H. M., "Atomic Dynamics of Explosive Boiling of LiquidArgon Films," Applied Physics B, 2005; 81: p. 5.

[96] Zou, Y., Huai, X. L., Lin, L., "Molecular Dynamics Simulation for Homogeneous Nucleation of Water and Liquid Nitrogen in Explosive Boiling," Applied Thermal Engineering, 2010; 30: p. 4. 
[97] Dotan, H., Kfir, O., Sharlin, E., Blank, O., Gross, M., Dumchin, I., Ankonina, G., Rothschild, A., "Resonant Light Trapping in Ultrathin Films for Water Splitting," Nature Materials, 2013; 12: p. 7.

[98] Khomenko, A. V., Prodanov, N. V., "Molecular Dynamics Simultaions of Ultrathin Water Film Confined between Flat Diamond Plates," Condensed Matter Physics, 2008; 4(56): p. 11.

[99] Yi, P., Poulikakos, D., Walther, J., Yadigaroglu, G., "Molecular Dynamics Simulation of Vaporization of an Ultra-Thin Liquid Argon Layer on a Surface," International Journal of Heat and Mass Transfer, 2002; 45: p. 14.

[100] Lin, C. P., Kelly, M. W., "Cavitation and Acoustic Emission around Laser-Heated Microparticles," Applied Physics Letters, 1998; 72(22): p. 3.

[101] Garmett, W. M., "Leidenfrost's Phenomenon," Nature, 1878: p. 1.

[102] Yu, J. P., and Wang, H., "A Molecular Dynamics Investigation on Evaporation of Thin Liquid Films," International Journal of Heat and Mass Transfer, 2012; 55: p. 8 .

[103] Adamson, A., Gast, A. Physical Chemistry of Surfaces. 6 ed: John Wiley and Sons Inc.; 1997.

[104] Hetsroni, G., Mosyak, A., Pogrebnyak, E., Yarin, L. P., "Heat Transfer in MicroChannel: Comparison of Experiments with Theory and Numerical Results," International Journal of Heat and Mass Transfer, 2005; 48: p. 22.

[105] Morini, G. L., "Single-Phase Convective Heat Transfer in Microchannels: A Review of Experimental Results," International Journal of Thermal Sciences, 2004; 43(7): p. 21. 
[106] Qwhaib, W., Palm, B., "Experimental Investigation of Single-Phase Convective Heat Transfer in Circular Microchannels," Experimental Thermal and Fluid Science, 2004; 28(2-3): p. 6.

[107] Cahill, D. G., Ford, W. K. , Goodson, K. E., Mahan, G. D. , Majumdar, A. , Maris, H. J. , Merlin, R., Phillpot, S. R. , "Nanoscale Thermal Transport," Applied Physics Review, 2003; 93(2): pp. 793-818.

[108] Liu, B., Jiang, H., Huang, Y., Qu, S., Yu, M. F., "Atomic-Scale Finite Element Method in Multiscale Computation with Applications to Carbon Nanotubes," Physical Review B, 2005; 72.

[109] Ganzenmuller, G. C., Hiermaier, S., Steinhauser, M. O., "Consistent Temperature Coupling with Thermal Fluctuations of Smooth Particle Hydrodynamics and Molecular Dynamics," PLoS ONE, 2012; 7(12): p. e51989.

[110] Zhou, W. J., Luan, H. B., Sun J.,He, Y. L.,Tao, W. Q., "Application of Fvm-Md Coupling Algorithm for Flow around Carbon Nanotube," Journal of Engineering Thermophysics, 2011; 32(4): pp. 679-682.

[111] Liu , G. R., Liu, M. B. Smoothed Particle Hydrodynamics: A Meshfree Particle Method. Singapore: World Scientific; 2003.

[112] Nie, X. B., Chen, S. Y., E, W. N. , Robbins, M. O. , "A Continuum and Molecular Dynamics Hybrid Method for Micro- and Nano-Fluid Flow," Journal Fluid Mechanics, 2004; 500: pp. 55-64.

[113] S. T. O'Connell, P. A. T., "Molecular Dynamics-Continuum Hybrid Computations: A Tool for Studying Complex Fuid Flow," Physics Review E, 1995: pp. R5792R5795. 
[114] Delgado-Buscalioni, R., Coveney, P.V. , "Continuum-Particle Hybrid Coupling for Mass, Momentum, and Energy Transfers in Unsteady Fuid Flow," Physics Review $E, 2003 ; 67,046704$.

[115] Werder, T., Walther, J. H. , Koumoutsakos, P. , "Hybrid Atomistic-Continuum Method for the Simulation of Dense Fluid Flows," Journal of Computational Physics, 2005; 205(1): pp. 373-390.

[116] Hadjiconstantinou, N. G., "Hybrid Atomistic-Continuum Formulations and the Moving Contact-Line Problem," Journal of Computational Physics, 1999; 154(2): pp. $245-265$.

[117] Borg, M. K., Macpherson, G. B., Reese, J. M. , "Controllers for Imposing Continuum-to-Molecular Boundary Conditions in Arbitrary Fluid Flow Geometries," Molecular Simulation, 2010; 36(10): pp. 745-757.

[118] Li, J., Liao, D. Y. , Yip, S "Coupling Continuum to Molecular-Dynamics Simulation: Reflecting Particle Method and the Field Estimator," Physical Review E, 1998; 57(6): pp. 7259-7267.

[119] Ren, W. Q., "Analytical and Numerical Study of Coupled Atomistic-Continuum Methods for Fluids," Journal of Computational Physics, 2007; 227(2): pp. 13531371.

[120] Flekkøy, E. G., Wagner, G. , Feder, J. , "Hybrid Model for Combined Particle and Continuum Dynamics," Europhysics Letters, 2000; 52(3): pp. 271-276.

[121] Barsky, S. D.-B., R. Coveney, P. V., "Comparison of Molecular Dynamics with Hybrid Continuum-Molecular Dynamics for a Single Tethered Polymer in a Solvent," Journal of Chemical Physics, 2004; 121, 2403-2411. 
[122] Yasuda, S., Yamamoto, R., "A Model for Hybrid Simulations of Molecular Dynamics and Computational Fluid Dynamics," Physics of Fluids, 2008; 20, 113101.

[123] Wang, Y. C., He, G. W., "A Dynamic Coupling Model for Hybrid AtomisticContinuum Computations," Chemical Engineering Science, 2007; 62: pp. 35743579 .

[124] Liu, J., Chen, S. Y., Nie, X. B., Robbins, M. O. , "A Continuum-Atomistic Simulation of Heat Transfer in Micro- and Nano-Flows," Journal of Computational Physics, 2007; 227: pp. 279-291.

[125] Sun, J., He, Y. L., Tao, W. Q., "Molecular Dynamics-Continuum Hybrid Simulation for Condensation of Gas Flow in Microchannel," Microfluid Nanofluid, 2009; 7: pp. 407-433.

[126] OpenCFD. User Guider; 2013.

[127] Plimpton, S., "Fast Parallel Algorithms for Short-Range Molecular Dynamics," Journal of Computation Physics, 1995; 117(1): pp. 1-19.

[128] Goniva, C., Kloss, C., Deen, N.G., Kuipers, J.A.M. ,Pirker, S. , "Influence of Rolling Friction Modelling on Single Spout Fluidized Bed Simulations," Particuology, 2012.

[129] Klemens, P. G., Williams, R. K. , "Thermal Conductivity of Metals and Alloys," International Metals Reviews, 1985; 31: pp. 197-215.

[130] Finnisa, M. W., Sinclaira , J. E. , "A Simple Empirical N-Body Potential for Transition Metals," Philosophical Magazine A, 1984; 50(1): pp. 45-55. 
[131] Schneider, T., Stoll, E. , "Molecular-Dynamics Study of a Three-Dimensional One-Component Model for Distortive Phase Transitions," Physical Review B, 1978; 17: pp. 1302-1322.

[132] Tuckerman, D. B., Pease, R. F. W., "High-Performance Heat Sinking for Vlsi," IEEE Electron Device Letter, 1981; 2(5): pp. 126-129.

[133] McGaughey, A. J. H., Kaviany, M. , "Thermal Conductivity Decomposition and Analysis Using Molecular Dynamics Simulations. Part I. Lennard-Jones Argon," International Journal of Heat and Mass Transfer 2004; 47: pp. 1799-1816.

[134] Kang, H., Zhang, Y., and Yang, M., "Molecular Dynamics Simulation of Thermal Conductivity of $\mathrm{Cu}-\mathrm{Ar}$ Nanofluid Using Eam Potential for $\mathrm{Cu}-\mathrm{Cu}$ Interactions," Applied Physics A: Materials Science \& Processing, 2011; 103(4): pp. 1001-1008.

[135] Hwang, H. J., Kwon, O. K. , Kang, J. W. , "Copper Nanocluster Diffusion in Carbon Nanotube," Solid State Communications, 2004; 129(11): pp. 687-690.

[136] Faghri, A., Zhang, Y. W., Howell, J. Advanced Heat and Mass Transfer. Columbia, MO, USA: Global Digital Press; 2010.

[137] Rapaport, D. C. The Art of Molecular Dynamics Simulation. Cambridge: Cambridge University Press; 2001.

[138] Haynes, W. M. Handbook of Chemistry and Physics, 91st Ed. . New York: Taylor \& Francis; 2010.

[139] Liu, C. W., Ko, H. S., Gau, C. . Heat Transfer - Theoretical Analysis, Experimental Investigations and Industrial Systems: InTech; 2011. 
[140] Kang, H. B., Zhang, Y. W., Yang, M., "Molecular Dynamics Simulation of Thermal Conductivity of $\mathrm{Cu}-\mathrm{Ar}$ Nanofluid Using Eam Potential for $\mathrm{Cu}-\mathrm{Cu}$ Interactions," Applied Physics A, 2011; 103(4): pp. 1001-1008. 


\section{VITA}

Yijin Mao was born in 1985, and completed his Bachelor degree study in Engineering Mechanics at College of Science and Master degree study in Agricultural Engineering, both from China Agricultural University in Beijing, mainland of China. He started his Ph.D. study in Department of Mechanical and Aerospace Engineering at University of Missouri-Columbia since 2010 Fall. 